\title{
4-Hydroxy Pyridones from Heterologous Expression and Cultivation of the Native Host
}

Wei-Yang Zhang, ${ }^{\dagger 1}$ Yue Zhong, ${ }^{\dagger 1}$ Yang Yu,,${ }^{*}$ Dan-Feng Shi, ${ }^{\ddagger}$ Hui-Yun Huang, ${ }^{\dagger}$ Xiao-Long Tang, ${ }^{\dagger}$ YongHeng Wang, ${ }^{\ddagger}$ Guo-Dong Chen, ${ }^{\ddagger}$ Hui-Ping Zhang,,${ }^{\S}$ Chen-Li Liu, ${ }^{\dagger}$ Dan Hu, ${ }^{\ddagger}$ Hao Gao, ${ }^{\ddagger}$ Xin-Sheng Yao ${ }^{\ddagger *}$

$\dagger$ Institute of Synthetic Biology, Shenzhen Institutes of Advanced Technology, Chinese Academy of Sciences, Shenzhen 518055, People's Republic of China.

Institute of Traditional Chinese Medicine \& Natural Products, College of Pharmacy and Guangdong Province Key Laboratory of Pharmacodynamic Constituents of TCM and New Drug Research, Jinan University, Guangzhou 510632, People’s Republic of China.

$\S$ RIKEN SPring-8 Center, Yokohama, Kanagawa 2300045, Japan 


\section{Content List}

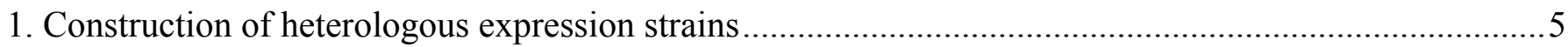

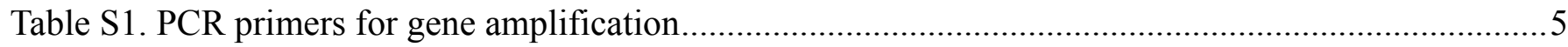

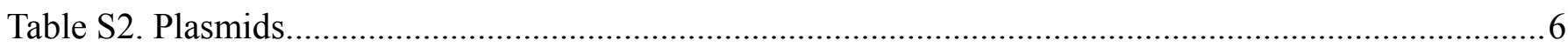

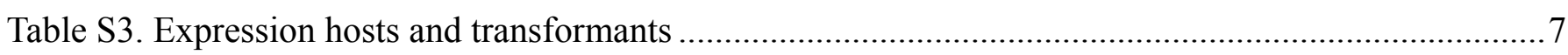

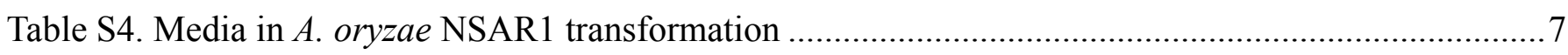

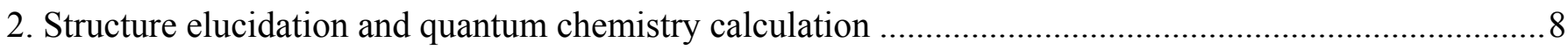

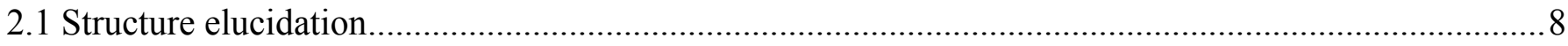

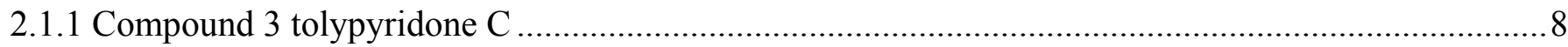

Table S5. NMR data of compound 3 (in DMSO- $d_{6}, 600 \mathrm{MHz}$ for ${ }^{1} \mathrm{H}$ and $150 \mathrm{MHz}$ for ${ }^{13} \mathrm{C}, J$ in $\mathrm{Hz}$ ) $\ldots \ldots . . .9$

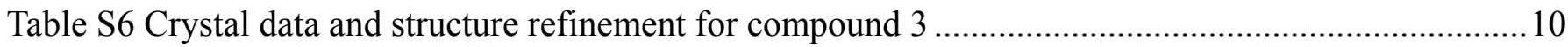

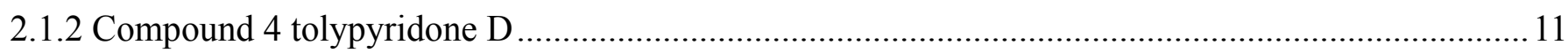

Table S7. NMR data of compound 4 (in DMSO-d6, $600 \mathrm{MHz}$ for ${ }^{1} \mathrm{H}$ and $150 \mathrm{MHz}$ for ${ }^{13} \mathrm{C}, J$ in $\mathrm{Hz}$ ) ....... 11

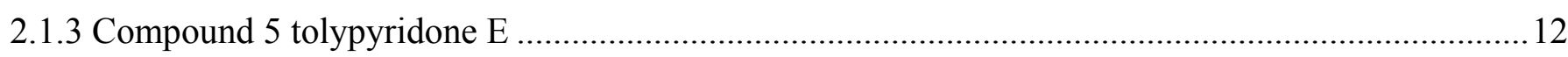

Table S8. NMR data of compound 5 (in DMSO-d6, $600 \mathrm{MHz}$ for ${ }^{1} \mathrm{H}$ and $150 \mathrm{MHz}$ for ${ }^{13} \mathrm{C}, J$ in $\mathrm{Hz}$ ) .......13

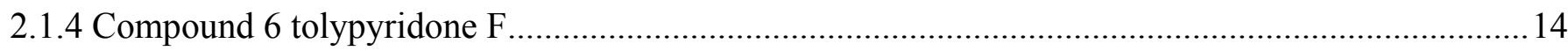

Table S9. NMR data of compound 6 (in DMSO- $d_{6}, 600 \mathrm{MHz}$ for ${ }^{1} \mathrm{H}$ and $150 \mathrm{MHz}$ for ${ }^{13} \mathrm{C}, J$ in $\mathrm{Hz}$ ) ...... 15

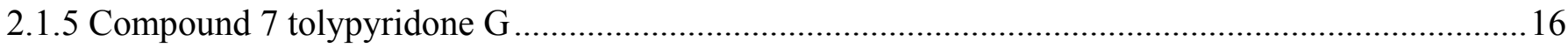

Table S10. NMR data of compound 7 (in DMSO- $d_{6}, 600 \mathrm{MHz}$ for ${ }^{1} \mathrm{H}$ and $150 \mathrm{MHz}$ for ${ }^{13} \mathrm{C}, J \mathrm{in} \mathrm{Hz}$ ) $\ldots . .16$

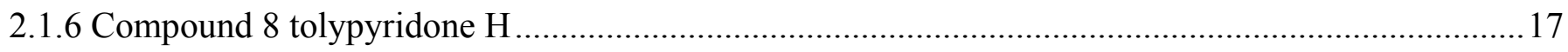

Table S11. NMR data of compound 8 (in DMSO-d6, $600 \mathrm{MHz}$ for ${ }^{1} \mathrm{H}$ and $150 \mathrm{MHz}$ for ${ }^{13} \mathrm{C}, J$ in $\mathrm{Hz}$ ) .....18

Table S12. NMR data comparison of tolypoalbin (1) and F-14329 (2) with reported data (in $\mathrm{CDCl}_{3}, 600$

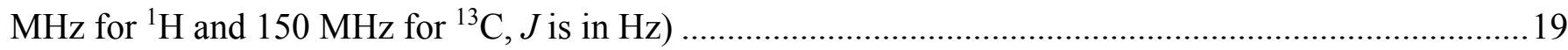

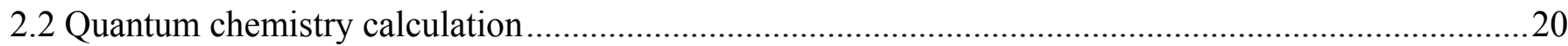

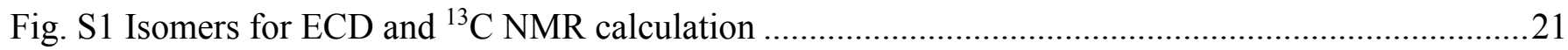

Table S13 Boltzmann distribution percentages of accepted conformers of a ........................................2

Table S14 Boltzmann distribution percentages of accepted conformers of $\mathrm{b}$..........................................2 21

Table S15 Boltzmann distribution percentages of accepted conformers of c ........................................21

Table S16 Boltzmann distribution percentages of accepted conformers of $\mathrm{d}$..........................................22

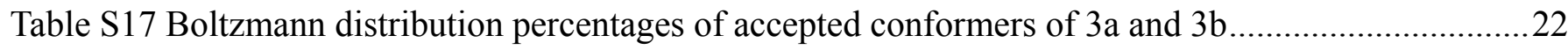

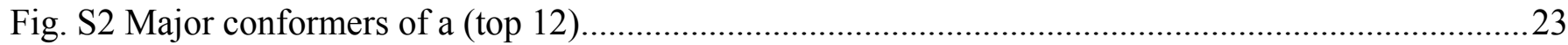

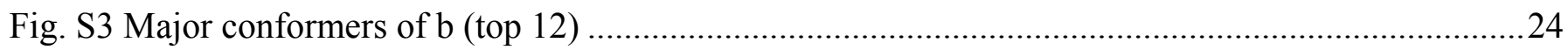

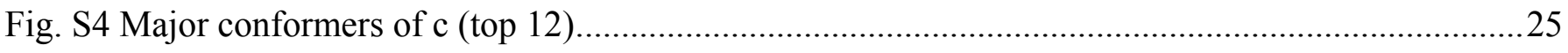

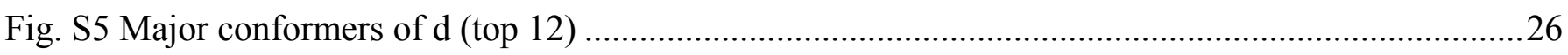

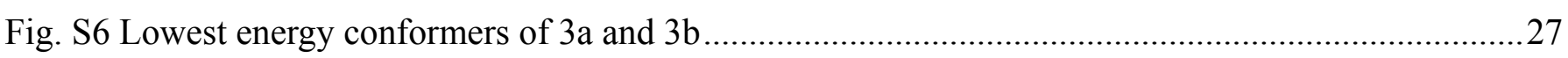

Table S18 Experimental ${ }^{13} \mathrm{C}$ NMR data of 5-8 and calculated ${ }^{13} \mathrm{C}$ NMR data of a-d ............................28

Table S19 R square $\left(\mathrm{R}^{2}\right)$ of the linear correlations and mean absolute error (MAE) values.....................28 
Table S2 ${ }^{13} \mathrm{C}$ NMR MAE $\Delta \Delta \delta$ values of the accounted comparison alignments of fixed sequence calc_a calc_b calc_c calc_d and all possible combinations of exp_5 exp_6 exp_7 exp_8 (the bold data set implies lowest $\mathrm{MAE}_{\Delta \Delta \delta}$ and the fittest sequence)

Fig. S7 Experimental and calculated CD spectra of compounds 5 and 7 (A), 6 and 8 (B).....................30

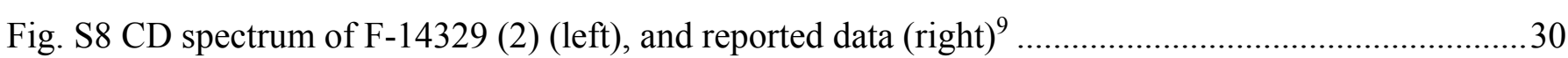

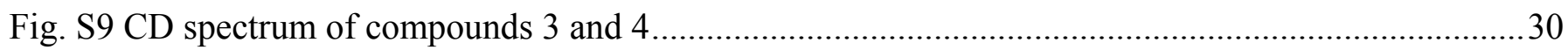

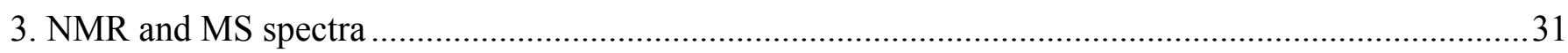

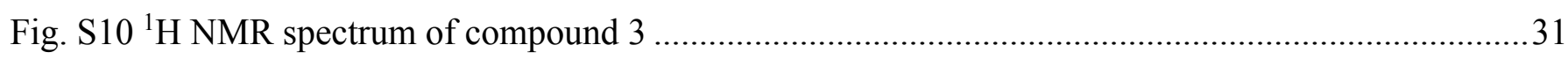

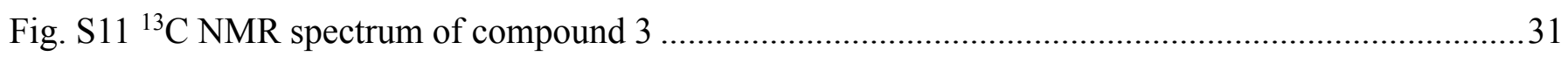

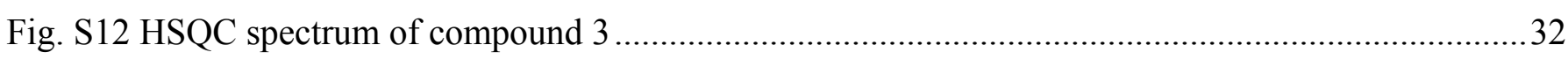

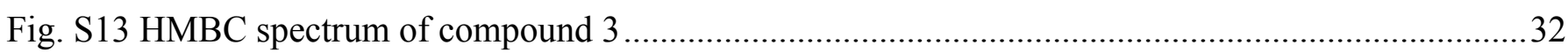

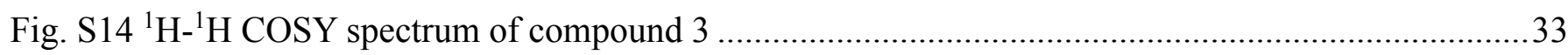

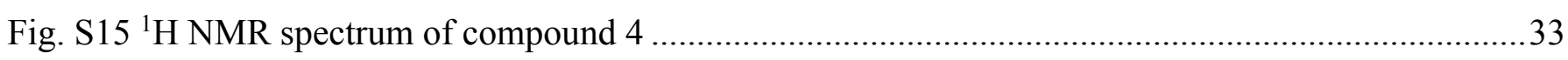

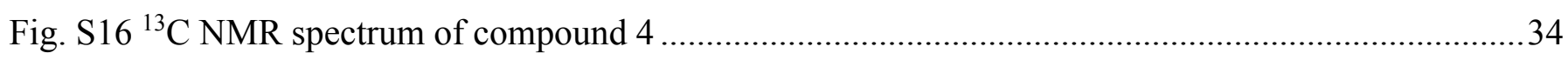

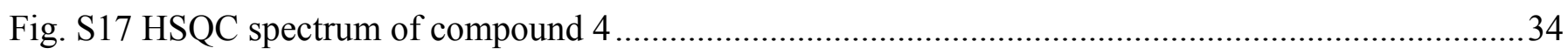

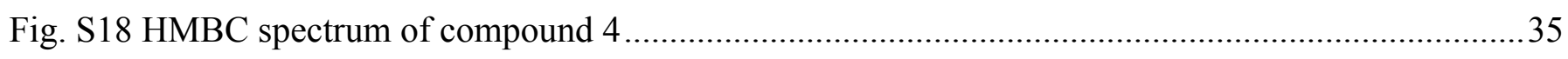

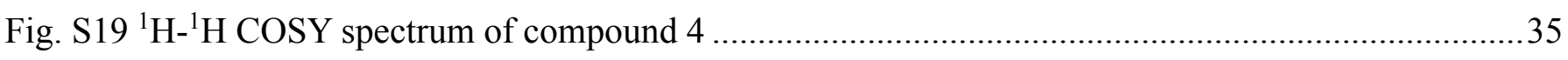

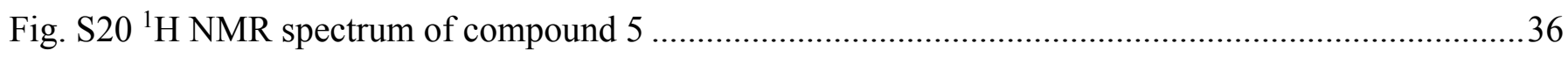

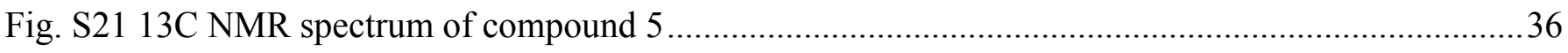

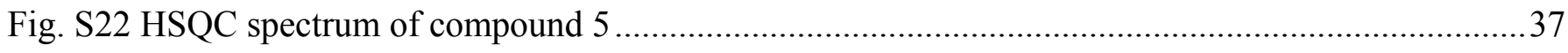

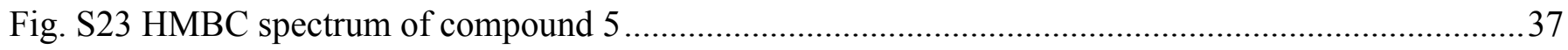

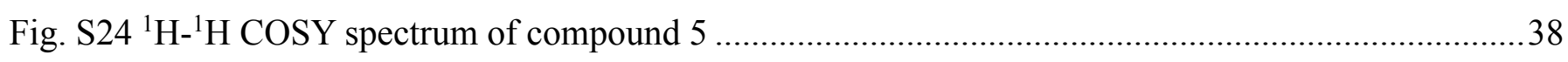

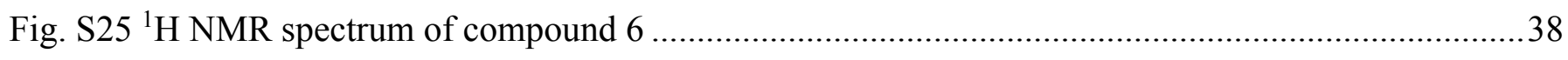

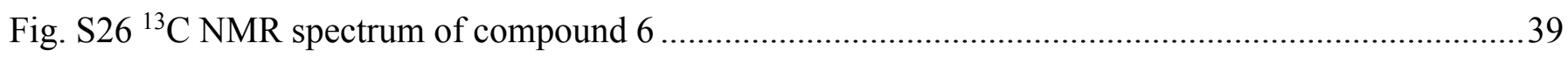

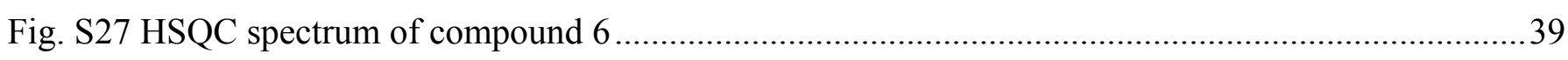

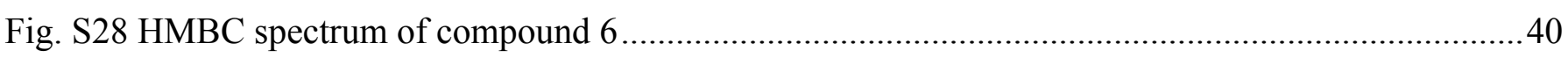

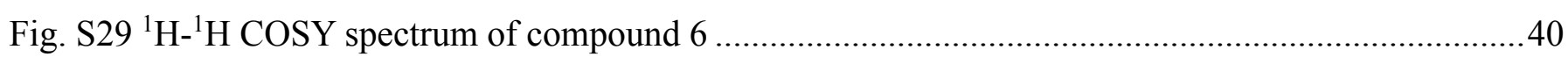

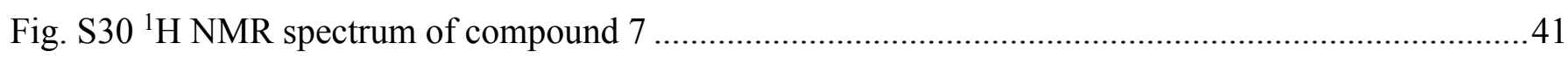

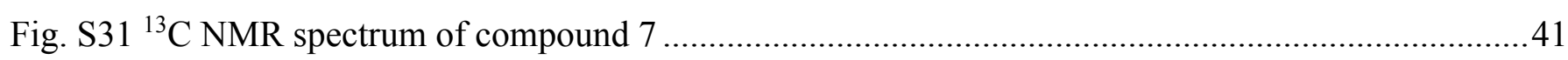

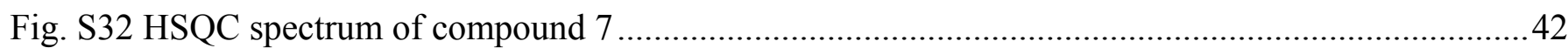

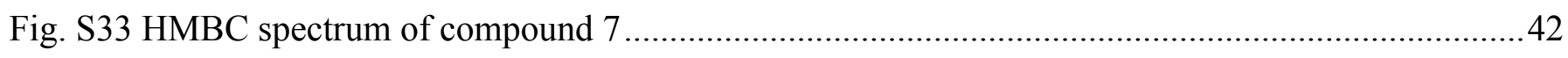

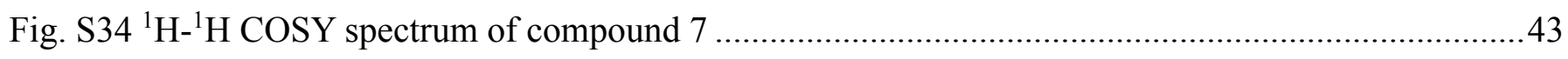

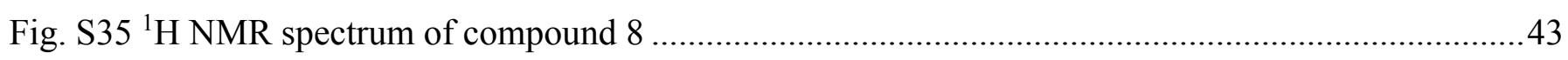

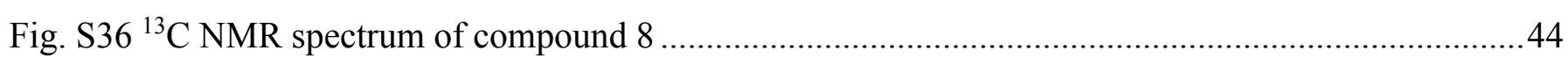

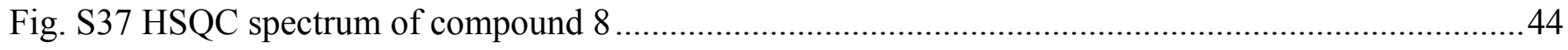

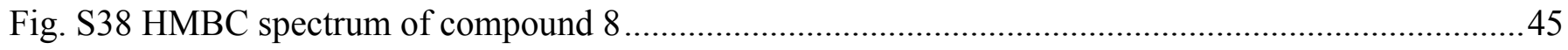

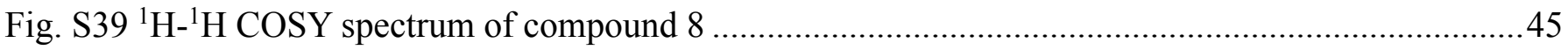


Fig. S40 HRESIMS report of compound 3

Fig. S41 HRESIMS report of compound 4 .47

Fig. S42 HRESIMS report of compound 5 .48

Fig. S43 HRESIMS report of compound 6 .49

Fig. S44 HRESIMS report of compound 7 .50

Fig. S45 HRESIMS report of compound 8 51

Fig. S46 HPLC-DAD-MS analysis of Tolypocladium sp. 49Y metabolites in different media .52

Fig. S47 Phylogenetic analysis of PKS-NRPSs encoded by four hit genes (PKS-NRPS) from Tolypocladium sp. 49Y. 


\section{Construction of heterologous expression strains}

Table S1. PCR primers for gene amplification

\begin{tabular}{|c|c|c|}
\hline Primers & Sequences $\left(5^{\prime}-3^{\prime}\right)$ & Usage \\
\hline g2334 F & TCGAGCTCGGTACCCACACACAATGTCCAAGCCAG & $\begin{array}{l}\text { Cloning tolA from } \\
\text { genomic DNA }\end{array}$ \\
\hline g2334 R & CTACTACAGATCCCCCCAACTCATTCCTCATTTAGC & $\begin{array}{c}\text { Cloning tolA from } \\
\text { genomic DNA }\end{array}$ \\
\hline$g 2335 \mathrm{~F}$ & TCGAGCTCGGTACCCACTTCGATGGGGAGCCTC & $\begin{array}{c}\text { Cloning tolC from } \\
\text { genomic DNA }\end{array}$ \\
\hline g2335 R & CTACTACAGATCCCCCGTCACAGGTCGTACACCAG & $\begin{array}{c}\text { Cloning tolC from } \\
\text { genomic DNA }\end{array}$ \\
\hline g2332 F & TCGAGCTCGGTACCCAAGATGGCCTTGGCACAGTG & $\begin{array}{l}\text { Cloning tolB from } \\
\text { genomic DNA }\end{array}$ \\
\hline g2332 R & CTACTACAGATCCCCCTCTAAGCCGGCGTGACCG & $\begin{array}{c}\text { Cloning tolB from } \\
\text { genomic DNA }\end{array}$ \\
\hline g2333 F & TCGAGCTCGGTACCCATGAACTCTACAGATCAAACTT & $\begin{array}{c}\text { Cloning tolD from } \\
\text { genomic DNA }\end{array}$ \\
\hline g2333 R & CTACTACAGATCCCCCTTACCAAAGTGTTGAGGCC & $\begin{array}{c}\text { Cloning tolD from } \\
\text { genomic DNA }\end{array}$ \\
\hline $\begin{array}{l}\text { Inf-pUSA- } \\
\text { Tamy-F }\end{array}$ & GAAAGGATCCTCTAGCGACTCCAATCTTCAAGAGC & $\begin{array}{c}\text { Cloning tolB }+ \text { amy } B \\
\text { from pTAex3-tolB } \\
\text { plasmid }\end{array}$ \\
\hline $\begin{array}{l}\text { Inf-pUSA- } \\
\text { Tamy-R }\end{array}$ & CCAAGATCGACTCTAGGTAAGATACATGAGCTTCGG & $\begin{array}{c}\text { Cloning tolB }+a m y B \\
\text { from pTAex3-tolB } \\
\text { plasmid }\end{array}$ \\
\hline $\begin{array}{c}\text { Inf-pAde-Parm- } \\
\text { F }\end{array}$ & GCAGGTCGACTCTAGACGACTCCAATCTTCAAGAGC & $\begin{array}{c}\text { Cloning tolD }+a m y B \\
\text { from pTAex3-tolD } \\
\text { plasmid }\end{array}$ \\
\hline $\begin{array}{c}\text { Inf-pAdeA- } \\
\text { Tamy-R-EcoRV }\end{array}$ & TAGTAGATCCTCTAGAGATATCGTAAGATACATGAGCTTCGG & $\begin{array}{c}\text { Cloning tolD }+a m y B \\
\text { from pTAex3-tolD } \\
\text { plasmid }\end{array}$ \\
\hline 2332 F EcoRV & CATGTATCTTACGATCCAATCTTCAAGAGCAGAAT & $\begin{array}{c}\text { Cloning tolB }+a m y B \\
\text { from pTAex3-tolB } \\
\text { plasmid }\end{array}$ \\
\hline $2332 \mathrm{R}$ EcoRV & AGATCCTCTAGAGATGTAAGATACATGAGCTTCGG & $\begin{array}{c}\text { Cloning tolB }+a m y B \\
\text { from pTAex3-tolB } \\
\text { plasmid }\end{array}$ \\
\hline
\end{tabular}


Table S2. Plasmids

Plasmids

Descriptions

Source

This plasmid contains an $a m y B$ promoter and an $\arg B$

pTAex3 marker gene cassette for gene expression in $A$. oryzae

Fujii et al. ${ }^{1}$ NSAR1.

This plasmid contains an $a m y B$ promoter and an $s C$ pUSA marker gene cassette for gene expression in $A$. oryzae NSAR1.

Yamada et al..$^{2}$

pAdeA

This plasmid contains an adeA marker gene cassette for gene expression in $A$. oryzae NSAR1.

Jin et al. ${ }^{3}$

pTAex3- tolA

This plasmid is pTAex 3 containing tolA and gene expression is regulated by $a m y B$ promoter.

This work

pUSA- tolC

This plasmid is pUSA containing tolC and gene expression is regulated by $a m y B$ promoter.

This work

This plasmid is pUSA containing tolB and tolC and gene expression is regulated by $a m y B$ promoter.

This work

pUSA- tolB- tolC

This plasmid is pAdeA containing tolB and tolD and

pAdeA- tolD- tolB gene expression is regulated by $a m y B$ promoter.

This work 
Table S3. Expression hosts and transformants

\begin{tabular}{clc}
\hline Strain & \multicolumn{1}{c}{ Description } & Source \\
\hline E. coli DH5a & Host for plasmid construction and amplification & TaKaRa \\
A. oryzae & A quadruple auxotrophic strain, host for BGC heterologous & \\
NSAR1 & expression & ${\text { Jin } \text { et }^{4}{ }^{4}}_{\text {AO1 }}^{\text {A. oryzae NSAR1 transformant with tolA and tolC }}$ \\
AO2 & A. oryzae NSAR1 transformant with tolA, tolB and tolC & This work \\
AO3 & A. oryzae NSAR1 transformant with tolA, tolB, tolC and tolD & This work \\
\hline
\end{tabular}

Table S4. Media in A. oryzae NSAR1 transformation

\begin{tabular}{|c|c|c|}
\hline Medium & Composition & Usage $^{5}$ \\
\hline DPY medium & $\begin{array}{l}\text { Dextrin } 2 \% \text {, polypeptone } 1 \% \text {, yeast extract } 0.5 \% \text {, } \\
\mathrm{MgSO}_{4} \cdot 7 \mathrm{H}_{2} \mathrm{O} 0.05 \%, \mathrm{KH}_{2} \mathrm{PO}_{4} 0.5 \% \text {, adenine } 0.01 \%\end{array}$ & $\begin{array}{l}\text { Pre-culturing of } A \text {. } \\
\text { oryzae NSAR1 } \\
\text { transformants }\end{array}$ \\
\hline $\begin{array}{l}\text { Czapek Dox - } \\
\text { starch }\end{array}$ & $\begin{array}{l}\mathrm{NaNO}_{3} 0.3 \%, \mathrm{KCl} 0.2 \% \text {, polypeptone } 1 \%, \mathrm{MgSO}_{4} \cdot 7 \mathrm{H}_{2} \mathrm{O} \\
0.05 \%, \mathrm{KH}_{2} \mathrm{PO}_{4} 0.1 \%, \mathrm{FeSO}_{4} \cdot 7 \mathrm{H}_{2} \mathrm{O} 0.002 \% \text {, adenine } \\
0.001 \% \text {, starch } 2 \%, \mathrm{pH} 5.5\end{array}$ & $\begin{array}{l}\text { Culturing } A \text {. oryzae } \\
\text { NSAR1 transformants }\end{array}$ \\
\hline Selective medium & $\begin{array}{l}0.2 \% \mathrm{NH}_{4} \mathrm{Cl}, 0.1 \%\left(\mathrm{NH}_{4}\right)_{2} \mathrm{SO}_{4}, 0.05 \% \mathrm{KCl}, 0.05 \% \mathrm{NaCl} \text {, } \\
0.1 \% \quad \mathrm{KH}_{2} \mathrm{PO}_{4}, 0.05 \% \quad \mathrm{MgSO}_{4} \cdot 7 \mathrm{H}_{2} \mathrm{O}, 0.002 \% \\
\mathrm{FeSO}_{4} \cdot 7 \mathrm{H}_{2} \mathrm{O}, 2 \% \text { glucose, } 1.2 \mathrm{M} \text { sorbitol, } 2 \% \text { agar in } \\
\text { bottom medium and } 1 \% \text { agar in cover medium, } \mathrm{pH} 5.5 ; \\
0.15 \% \text { methionine, } 0.1 \% \text { arginine and } 0.01 \% \text { adenine were } \\
\text { used as selection markers }\end{array}$ & $\begin{array}{l}\text { Transformant } \\
\text { selection }\end{array}$ \\
\hline $\begin{array}{l}\text { Stabilization } \\
\text { medium }\end{array}$ & $\begin{array}{l}0.2 \% \mathrm{NH}_{4} \mathrm{Cl}, 0.1 \%\left(\mathrm{NH}_{4}\right)_{2} \mathrm{SO}_{4}, 0.05 \% \mathrm{KCl}, 0.05 \% \mathrm{NaCl} \text {, } \\
0.1 \% \quad \mathrm{KH}_{2} \mathrm{PO}_{4}, \quad 0.05 \% \quad \mathrm{MgSO}_{4} \cdot 7 \mathrm{H}_{2} \mathrm{O}, \quad 0.002 \% \\
\mathrm{FeSO}_{4} \cdot 7 \mathrm{H}_{2} \mathrm{O}, 2 \% \text { glucose, } 2 \% \text { agar, } \mathrm{pH} 5.5 ; 0.15 \% \\
\text { methionine, } 0.1 \% \text { arginine and } 0.01 \% \text { adenine were added } \\
\text { as supplement when necessary. }\end{array}$ & $\begin{array}{l}\text { Transformant } \\
\text { stablization }\end{array}$ \\
\hline
\end{tabular}




\section{Structure elucidation and quantum chemistry calculation}

\subsection{Structure elucidation}

\subsubsection{Compound 3 tolypyridone $C$}

Tolypyridone C (3) was isolated as yellow acicular crystals (72\% methanol aqueous solution). Quasimolecular ion at $\mathrm{m} / z 356.1870[\mathrm{M}+\mathrm{H}]^{+}$was indicative of molecular formula of $\mathrm{C}_{21} \mathrm{H}_{25} \mathrm{NO}_{4}$ (calculated for $\left.\mathrm{C}_{21} \mathrm{H}_{26} \mathrm{NO}_{4}, 356.1862\right) .{ }^{1} \mathrm{H}$ NMR and ${ }^{13} \mathrm{C} \mathrm{NMR}$ provided structural information of three methyl groups $\left(\delta_{\mathrm{H}}\right.$ $1.61, \delta_{\mathrm{C}} 18.0, \mathrm{CH}_{3}-10 ; \delta_{\mathrm{H}} 0.80, \delta_{\mathrm{C}} 19.6, \mathrm{CH}_{3}-11$; and $\left.\delta_{\mathrm{H}} 1.08, \delta \mathrm{C} 17.8, \mathrm{CH}_{3}-12\right)$, two methenes $\left(\delta_{\mathrm{H}} 1.80,1.07\right.$, $\delta_{\mathrm{C}} 39.9, \mathrm{CH}_{2}-5$ and $\left.\delta_{\mathrm{H}} 1.99,1.76, \delta_{\mathrm{C}} 39.5, \mathrm{CH}_{2}-7\right)$, two sp ${ }^{3}$ methines $\left(\delta_{\mathrm{H}} 4.46, \delta_{\mathrm{C}} 39.2, \mathrm{CH}-4 ; \delta_{\mathrm{H}} 1.41, \delta_{\mathrm{C}} 30.6\right.$, $\mathrm{CH}-6)$, olefinic and enoyl protons and carbons $\left(\delta_{\mathrm{H}} 7.56, \delta_{\mathrm{C}} 140.9, \mathrm{CH}-1^{\prime} ; \delta_{\mathrm{C}} 112.4, \mathrm{C}-3^{\prime} ; \delta_{\mathrm{H}} 5.38, \delta_{\mathrm{C}} 129.6\right.$, $\left.\mathrm{CH}-8 ; \delta_{\mathrm{H}} 5.38, \delta_{\mathrm{C}} 125.6, \mathrm{CH}-9 ; \delta_{\mathrm{C}} 106.1, \mathrm{C}-2 ; \delta_{\mathrm{C}} 175.9, \mathrm{C}-2^{\prime}\right)$, two carbonyl groups $\left(\delta_{\mathrm{C}} 161.3, \mathrm{C}-1 ; \delta_{\mathrm{C}} 212.2\right.$, $\mathrm{C}-3)$, and a set of typical para-hydroxyl phenyl group signals $\left(\delta \mathrm{C} 114.9, \mathrm{C}-4^{\prime} ; \delta_{\mathrm{H}} 7.25, \delta \mathrm{C} 140.9, \mathrm{CH}-5^{\prime}\right.$ and $9^{\prime}$; $\delta_{\mathrm{H}} 6.78, \delta_{\mathrm{C}} 130.1, \mathrm{CH}-6^{\prime}$ and $\left.8^{\prime} ; \delta_{\mathrm{C}} 156.7, \mathrm{C}-7^{\prime}\right)$. Data were unambiguously assigned by HSQC and HMBC spectra. Based on $\mathrm{HMBC}$ correlations from $\mathrm{H}_{3}-10$ to $\mathrm{C}-8 / 9$, from $\mathrm{H}-8$ to $\mathrm{C}-6 / 7$, from $\mathrm{H}_{3}-11$ to $\mathrm{C}-5 / 6 / 7$, and from $\mathrm{H}_{3}-12$ to $\mathrm{C}-3 / 4 / 5$, a substructure was proposed as a $\mathrm{C}_{8}$ chain with two methyl groups at $\mathrm{C}-4,6$ and a double bond at $\mathrm{C}-8,9$, and was confirmed by ${ }^{1} \mathrm{H}-{ }^{1} \mathrm{H}$ COSY correlations. HMBC correlations from $\mathrm{H}-2$ ' to C$1^{\prime} / 3^{\prime} / 1$, chemical shifts of $\mathrm{H}-2^{\prime}\left(\delta_{\mathrm{H}} 7.56\right),-\mathrm{NH}\left(\delta_{\mathrm{H}} 11.67\right), \mathrm{C}-1\left(\delta_{\mathrm{C}} 161.3\right)$ and $\mathrm{C}-2^{\prime}\left(\delta_{\mathrm{C}} 175.9\right)$ indicated the presence of a 4-hydroxy-2-pyridone substructure. Linkage between C-3' of the pyridone ring and C-4' of the phenyl group was established by HMBC correlation from $\mathrm{H}-1^{\prime}$ to $\mathrm{C}-4^{\prime}$ and from $\mathrm{H}-5^{\prime}$ to $\mathrm{C}-3^{\prime}$. Summing up the molecular formula of all above substructures, the total was equal to that given by HRMS, and based on the above analysis of NMR, the planar structure of $\mathbf{3}$ was established. According to X-ray single-crystal diffraction results (Fig. 2B), C-8(9) double bond was assigned trans, and relative configurations of C-4 and C-6 were assigned as $4 S^{*}, 6 S^{*}$. Comparison of experimental CD spectrum to calculated CD data of $4 S, 6 S-3$ and $4 R, 6 R-$ 3 (Fig. 2C) indicated that absolute configuration of this compound was $4 S, 6 S$. Thus, this compound was identified and named as tolypyridone $\mathrm{C}$.

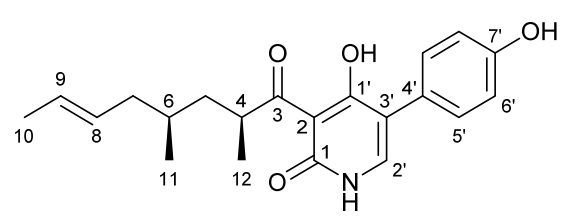

3

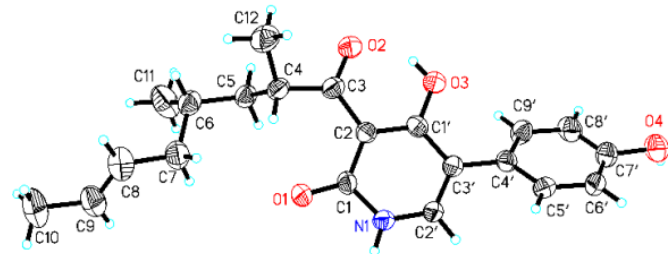


Table S5. NMR data of compound 3 (in DMSO- $d_{6}, 600 \mathrm{MHz}$ for ${ }^{1} \mathrm{H}$ and $150 \mathrm{MHz}$ for ${ }^{13} \mathrm{C}, J$ in $\mathrm{Hz}$ )

\begin{tabular}{|c|c|c|c|c|}
\hline Pos. & $\delta_{\mathrm{H}}$ & $\delta \mathrm{C}$ & ${ }^{1} \mathrm{H}-{ }^{1} \mathrm{H} \mathrm{COSY}$ & $\mathrm{HMBC}$ \\
\hline 1 & - & 161.3 & & \\
\hline 2 & - & 105.4 & & \\
\hline 3 & - & 212.2 & & \\
\hline 4 & 4.46 (sextet, 7.0$)$ & 39.2 & $5 \mathrm{a}, 5 \mathrm{~b}, 12$ & $3,5,6,12$ \\
\hline \multirow{2}{*}{5} & & \multirow{2}{*}{39.9} & $4,5 b, 6$ & $3,4,6,7,11,12$ \\
\hline & $1.07, \mathrm{~b}$ & & $4,5 a, 6$ & $3,4,6,7,11,12$ \\
\hline 6 & 1.41 & 30.6 & $5 \mathrm{a}, 5 \mathrm{~b}, 7 \mathrm{a}, 7 \mathrm{~b}, 11$ & $5,7,8,11$ \\
\hline \multirow{2}{*}{7} & 1.99, a & \multirow{2}{*}{39.5} & $6,7 b, 8$ & $6,8,9,11$ \\
\hline & $1.76, \mathrm{~b}$ & & $6,7 a, 8$ & $6,8,9,11$ \\
\hline 8 & 5.38 & 129.6 & 7 & $6,7,9$ \\
\hline 9 & 5.38 & 125.6 & 10 & $7,8,10$ \\
\hline 10 & $1.61(\mathrm{dd}, 3.4,0.7)$ & 18.0 & 8,9 & 8,9 \\
\hline 11 & $0.80(\mathrm{~d}, 6.7)$ & 19.6 & 6 & $5,6,7$ \\
\hline 12 & $1.08(\mathrm{~d}, 6.7)$ & 17.8 & 4 & $3,4,5$ \\
\hline $1^{\prime}$ & - & 175.9 & & \\
\hline $2^{\prime}$ & 7.56 (br s) & 140.9 & $=\mathrm{NH}$ & $2,1^{\prime}, 3^{\prime}, 4^{\prime}, 1$ \\
\hline $3^{\prime}$ & - & 112.4 & & \\
\hline $4^{\prime}$ & - & 123.3 & & \\
\hline $5^{\prime} / 9^{\prime}$ & $7.25(\mathrm{~d}, 8.6)$ & 130.1 & $6^{\prime}, 8^{\prime}$ & $2^{\prime}, 3^{\prime}, 6^{\prime}, 7^{\prime}, 8^{\prime}$ \\
\hline $6^{\prime} / 8^{\prime}$ & $6.78(\mathrm{~d}, 8.6)$ & 114.9 & $5^{\prime}, 9^{\prime}$ & $4^{\prime}, 5^{\prime}, 7^{\prime}, 9^{\prime}$ \\
\hline $7^{\prime}$ & - & 156.7 & & \\
\hline $7^{\prime}-\mathrm{OH}$ & 9.49 (br s) & & & $6^{\prime}, 7^{\prime}, 8^{\prime}$ \\
\hline$=\mathrm{NH}$ & 11.67 (br s) & & $2^{\prime}$ & \\
\hline
\end{tabular}

${ }^{a}$ Multiplets and overlapped signals are given without assigning multiplicity. 


\begin{tabular}{|c|c|}
\hline Item & Value \\
\hline Identification code & $3 \mathrm{G} 6$ \\
\hline Empirical formula & $\mathrm{C}_{21} \mathrm{H}_{25} \mathrm{NO}_{4}$ \\
\hline Formula weight & 355.42 \\
\hline Temperature/K & 293(2) \\
\hline Crystal system & monoclinic \\
\hline Space group & $\mathrm{P} 2{ }_{1}$ \\
\hline $\mathrm{a} / \AA$ & $14.8720(7)$ \\
\hline $\mathrm{b} / \AA$ & $7.8414(5)$ \\
\hline $\mathrm{c} / \AA$ & $35.3848(17)$ \\
\hline$\alpha /^{\circ}$ & 90 \\
\hline$\beta /{ }^{\circ}$ & $92.297(4)$ \\
\hline$\gamma /{ }^{\circ}$ & 90 \\
\hline Volume $/ \AA^{3}$ & $4123.2(4)$ \\
\hline $\mathrm{Z}$ & 8 \\
\hline$\rho_{\text {calcg }} / \mathrm{cm}^{3}$ & 1.145 \\
\hline$\mu / \mathrm{mm}^{-1}$ & 0.639 \\
\hline $\mathrm{F}(000)$ & 1520.0 \\
\hline Crystal size $/ \mathrm{mm}^{3}$ & $0.14 \times 0.12 \times 0.11$ \\
\hline Radiation & $\mathrm{Cu} \mathrm{K \alpha}(\lambda=1.54184)$ \\
\hline $2 \Theta$ range for data collection $/{ }^{\circ}$ & 4.998 to 148.136 \\
\hline Index ranges & $-17 \leqslant \mathrm{~h} \leqslant 18,-9 \leqslant \mathrm{k} \leqslant 5,-37 \leqslant 1 \leqslant 43$ \\
\hline Reflections collected & 16490 \\
\hline Independent reflections & $11071\left[\mathrm{R}_{\mathrm{int}}=0.0632, \mathrm{R}_{\mathrm{sigma}}=0.0752\right]$ \\
\hline Data/restraints/parameters & $11071 / 22 / 940$ \\
\hline Goodness-of-fit on $\mathrm{F}^{2}$ & 0.985 \\
\hline Final $R$ indexes $[\mathrm{I}>=2 \sigma(\mathrm{I})]$ & $\mathrm{R}_{1}=0.0667, \mathrm{wR}_{2}=0.1580$ \\
\hline Final $\mathrm{R}$ indexes [all data] & $\mathrm{R}_{1}=0.1051, \mathrm{wR}_{2}=0.1912$ \\
\hline Largest diff. peak/hole / e $\AA^{-3}$ & $0.21 /-0.21$ \\
\hline Flack parameter & $0.2(3)$ \\
\hline
\end{tabular}




\subsubsection{Compound 4 tolypyridone D}

Tolypyridone D (4) was isolated as light yellow amorphous gum, HR-ESI-MS showed a quasi-molecular ion at $\mathrm{m} / z 264.1595[\mathrm{M}+\mathrm{H}]^{+}$, which was indicative of molecular formula $\mathrm{C}_{15} \mathrm{H}_{21} \mathrm{NO}_{3}$ (calculated for $\left.\mathrm{C}_{15} \mathrm{H}_{22} \mathrm{NO}_{3}, 264.1600\right) .{ }^{1} \mathrm{H} \mathrm{NMR}$ and ${ }^{13} \mathrm{C} \mathrm{NMR}$ showed three groups of methyl signals $\left(\delta_{\mathrm{H}} 1.04, \delta_{\mathrm{C}} 17.8, \mathrm{CH}_{3}-\right.$ $\left.12 ; \delta_{\mathrm{H}} 0.78, \delta_{\mathrm{C}} 19.6, \mathrm{CH}_{3}-11 ; \delta_{\mathrm{H}} 1.61, \delta_{\mathrm{C}} 17.9, \mathrm{CH}_{3}-10\right)$, two methenes $\left(\delta_{\mathrm{H}} 1.95,1.75, \delta_{\mathrm{C}} 39.5, \mathrm{CH}_{2}-7 ; \delta_{\mathrm{H}} 1.78\right.$, $\left.1.03, \delta_{\mathrm{C}} 39.8, \mathrm{CH}_{2}-5\right)$, two sp ${ }^{3}$ methines $\left(\delta_{\mathrm{H}} 1.38, \delta_{\mathrm{C}} 30.6, \mathrm{CH}-6 ; \delta_{\mathrm{H}} 4.34, \delta_{\mathrm{C}} 39.3, \mathrm{CH}-4\right)$, two pairs of double bonds $\left(\delta_{\mathrm{H}} 5.93, \delta_{\mathrm{C}} 99.2, \mathrm{CH}-3{ }^{\prime} ; \delta_{\mathrm{H}} 7.60, \delta_{\mathrm{C}} 142.8, \mathrm{CH}-1 ' ; \delta_{\mathrm{H}} 5.36, \delta_{\mathrm{C}} 129.5, \mathrm{CH}-8 ; \delta_{\mathrm{H}} 5.36, \delta_{\mathrm{C}} 125.6, \mathrm{CH}-9\right)$, and four quaternary carbons including two carbonyl groups and a pair of enol carbons $\left(\delta_{\mathrm{C}} 106.1, \mathrm{C}-2 ; \delta_{\mathrm{C}} 161.9\right.$, $\left.\mathrm{C}-1 ; \delta_{\mathrm{C}} 177.6, \mathrm{C}-2{ }^{\prime} ; \delta_{\mathrm{C}} 211.7, \mathrm{C}-3\right)$. Data were unambiguously assigned based HSQC and HMBC spectra. Based on HMBC correlations from $\mathrm{H}_{3}-10$ to C-8/C-9, from $\mathrm{H}-8$ to C-6/C-7, from $\mathrm{H}_{3}-11$ to C-5/C-6/C-7, and from $\mathrm{H}_{3}-12$ to $\mathrm{C}-3 / \mathrm{C}-4 / \mathrm{C}-5$, a substructure was proposed as a $\mathrm{C}_{8}$ chain with two methyl groups at $\mathrm{C}-4$ and 6 and a double bond at C-8(9), and was verified by ${ }^{1} \mathrm{H}-{ }^{1} \mathrm{H}$ COSY correlation peaks. The double bond was identified as trans- by chemical shifts of C-7 and C-10 ${ }^{6}$ and referring to tolypyridone C (3). HMBC correlations from $\mathrm{H}-2^{\prime}$ to $\mathrm{C}-1^{\prime} / \mathrm{C}-3^{\prime} / \mathrm{C}-1$, and from $\mathrm{H}-3^{\prime}$ to $\mathrm{C}-2 / \mathrm{C}-1^{\prime} / \mathrm{C}-2^{\prime}$, and ${ }^{1} \mathrm{H}-{ }^{1} \mathrm{H}$ COSY correlation between $\mathrm{H}-1^{\prime}$ and $\mathrm{H}-3^{\prime}$ indicated the presence of a 4-hydroxy-2-pyridone substructure. HMBC correlation from $\mathrm{H}-4$ to C-2 linked the octanoyl substructure to C-2 position of the pyridone ring, and the whole planar structure of this compound was identified. The configuration of $\mathbf{4}$ was deduced as $4 S, 6 S$ by referring to its biosynthetic cogener, tolypyridone C (3), and this deduction was confirmed the fact that CD spectrum of $\mathbf{4}$ showed similar Cotton effects with 3 (Fig. S22).

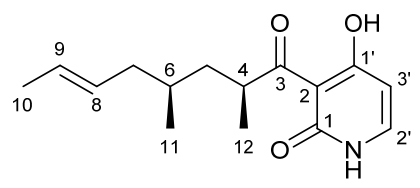

4

Table S7. NMR data of compound 4 (in DMSO- $d_{6}, 600 \mathrm{MHz}$ for ${ }^{1} \mathrm{H}$ and $150 \mathrm{MHz}$ for ${ }^{13} \mathrm{C}, J$ in $\mathrm{Hz}$ )

\begin{tabular}{ccccc}
\hline Pos. & $\delta_{\mathrm{H}}$ & $\delta_{\mathrm{C}}$ & ${ }^{1} \mathrm{H}-{ }^{1} \mathrm{H}$ COSY & $\mathrm{HMBC}$ \\
\hline 1 & - & 161.9 & & \\
2 & - & 106.1 & & \\
3 & - & 211.7 & & $3,5,6,12$ \\
4 & 4.34 (sextet, 7.0) & 39.3 & $5 \mathrm{a}, 5 \mathrm{~b}, 12$ & $3,6,7,10,12$ \\
5 & $1.78, \mathrm{a}$ & & $4,5 \mathrm{~b}, 6$ & $3,6,7,10,12$ \\
6 & $1.03, \mathrm{~b}$ & 39.8 & $4,5 \mathrm{a}, 6$ & $5,8,11$ \\
7 & 1.38 & 30.6 & $5 \mathrm{a}, 5 \mathrm{~b}, 7 \mathrm{a}, 7 \mathrm{~b}, 11$ & $5,6,8,9,11$ \\
\hline
\end{tabular}




\begin{tabular}{ccccc} 
& $1.75, \mathrm{~b}$ & & $6,7 \mathrm{a}, 8$ & $5,6,8,9,11$ \\
8 & 5.36 & 129.5 & 7 & $6,7,9$ \\
9 & 5.36 & 125.6 & 10 & $7,8,10$ \\
10 & $1.61(\mathrm{dd}, 3.4$, & 17.9 & 9 & $6,8,9$ \\
11 & $0.7)$ & & & $5,6,7$ \\
12 & $1.04(\mathrm{~d}, 6.7)$ & 17.8 & 4 & $3,4,5$ \\
$1^{\prime}$ & - & 177.6 & & $1,1^{\prime}, 3^{\prime}$, \\
$2^{\prime}$ & $7.60(\mathrm{br} \mathrm{d}, 7.2)$ & 142.8 & $3^{\prime},=\mathrm{NH}$ & $2,3,1^{\prime}, 2^{\prime}$ \\
$3^{\prime}$ & $5.93(\mathrm{~d}, 7.2)$ & 99.2 & $2^{\prime}$ & \\
$=\mathrm{NH}$ & $11.51(\mathrm{br}$ s $)$ & & $2^{\prime}$ & \\
\hline
\end{tabular}

${ }^{a}$ Multiplets and overlapped signals are given without assigning multiplicity.

\subsubsection{Compound 5 tolypyridone $\mathbf{E}$}

Tolypyridone E (5) was isolated as light yellow amorphous powder, quasi-molecular ion given by HRESI-MS was $m / z 356.1860[\mathrm{M}+\mathrm{H}]^{+}$, indicative of molecular formula $\mathrm{C}_{21} \mathrm{H}_{25} \mathrm{NO}_{4}\left([\mathrm{M}+\mathrm{H}]^{+}\right.$calcd for $\left.\mathrm{C}_{21} \mathrm{H}_{26} \mathrm{NO}_{4}, 356.1862\right) .{ }^{1} \mathrm{H}$ NMR and ${ }^{13} \mathrm{C}$ NMR showed three methyl groups $\left(\delta_{\mathrm{H}} 0.88, \delta_{\mathrm{C}} 21.5, \mathrm{CH}_{3}-11 ; \delta_{\mathrm{H}}\right.$ $\left.1.37, \delta_{\mathrm{C}} 18.9, \mathrm{CH}_{3}-12 ; \delta_{\mathrm{H}} 1.52, \delta_{\mathrm{C}} 17.7, \mathrm{CH}_{3}-10\right)$, two methenes $\left(\delta_{\mathrm{H}} 1.61\right.$ and $1.28, \delta_{\mathrm{C}} 44.2, \mathrm{CH}_{2}-5 ; \delta_{\mathrm{H}} 1.89$ and $\left.1.78, \delta_{\mathrm{C}} 40.7, \mathrm{CH}_{2}-7\right)$, two $\mathrm{sp}^{3}$ methine ( $\left.\delta_{\mathrm{H}} 4.58, \delta \mathrm{C} 74.2, \mathrm{CH}-3 ; \delta_{\mathrm{H}} 1.66, \delta \mathrm{C} 28.6, \mathrm{CH}-6\right)$, three exchangeable protons $\left(\delta_{\mathrm{H}} 5.22,3-\mathrm{OH} ; \delta_{\mathrm{H}} 9.45,7^{\prime}-\mathrm{OH} ; \delta_{\mathrm{H}} 11.26,=\mathrm{NH}\right)$, one sp ${ }^{3}$ quaternary carbon $\left(\delta_{\mathrm{C}} 94.3, \mathrm{C}-4\right)$, two olefinic carbons $\left(\delta_{\mathrm{H}} 5.25, \delta_{\mathrm{C}} 129.3, \mathrm{CH}-8 ; \delta_{\mathrm{H}} 5.25, \delta_{\mathrm{C}} 126.1, \mathrm{CH}-9\right)$, a carboxyl signal $\left(\delta_{\mathrm{C}} 160.5, \mathrm{C}-1\right)$, and ten other $\mathrm{sp}^{2}$ carbon and related proton signals $\left(\delta_{\mathrm{C}} 165.2, \mathrm{C}-1^{\prime} ; \delta_{\mathrm{H}} 7.39, \delta \mathrm{C} 134.3, \mathrm{CH}-2^{\prime} ; \delta_{\mathrm{C}} 108.4, \mathrm{C}-3^{\prime} ; \delta_{\mathrm{C}} 123.9, \mathrm{C}-4^{\prime} ;\right.$ $\delta_{\mathrm{H}} 7.33, \delta_{\mathrm{C}} 128.3, \mathrm{CH}-5^{\prime}$ and $9^{\prime} ; \delta_{\mathrm{H}} 6.76, \delta_{\mathrm{C}} 115.2, \mathrm{CH}-6^{\prime}$ and $\left.8^{\prime} ; \delta_{\mathrm{C}} 156.4, \mathrm{C}-7^{\prime}\right)$. NMR signals were unambiguously assigned according to HSQC and HMBC spectra. The aromatic signals were typical of a parasubstituted phenyl ring. HMBC correlations from $\mathrm{H}-3$ to $\mathrm{C}-1 / \mathrm{C}-2 / \mathrm{C}-3$, and from $\mathrm{H}-2^{\prime}$ to $\mathrm{C}-1 / \mathrm{C}-2 / \mathrm{C}-1^{\prime} / \mathrm{C}-3^{\prime} / \mathrm{C}-$ 4 ' along with the chemical shifts of C-1, CH-2, C-3 and N-H indicated the presence of a pyridone ring. HMBC correlations from $\mathrm{H}-5^{\prime} / 9^{\prime}$ to $\mathrm{C}-3^{\prime}$ and $\mathrm{H}-2^{\prime}$ to $\mathrm{C}-4^{\prime}$ linked the phenyl ring to the pyridone to establish a phenyl pyridone substructure. $\mathrm{A}_{6}$ chain with a double bond next to its terminal was revealed by $\mathrm{HMBC}$ correlations from $\mathrm{H}-8$ and $\mathrm{H}-9$ to $\mathrm{C}-7 / \mathrm{C}-10$, and from $\mathrm{H}-6$ to $\mathrm{C}-5 / \mathrm{C}-7 / \mathrm{C}-8$. The double bond was identified as trans according to chemical shifts of C-7 and C-10, ${ }^{6}$ and this was confirmed by referring to its congener, compound 3. HMBC correlations from the methyl proton $\mathrm{H}_{3}-11$ to $\mathrm{C}-4 / \mathrm{C}-5 / \mathrm{C}-6$ linked the methyl $\mathrm{C}-11$ to the chain. HMBC correlations from the other methyl proton signal $\mathrm{H}_{3}-12$ to $\mathrm{C}-3 / \mathrm{C}-4 / \mathrm{C}-5$, and from $\mathrm{H}_{2}-5$ to $\mathrm{C}-3 / \mathrm{C} 4$, and 3-OH to C-3 indicated that the carbon chain and a methyl group $\mathrm{CH}_{3}-12$ was linked to oxygenated quaternary C-4 and a hydroxyl group was at C-3. Next, HMBC correlations from H-3 to C-1/C-2/C-1' indicated C-3 was 
linked to the pyridone ring at C-2. All substructures possessed 9 degrees of unsaturation, one less than the proposed 10 degrees of unsaturation. Based on unsaturation indices and carbon chemical shifts of C-1' and C$4,{ }^{5}$ oxygenated $\mathrm{C}-4$ was linked to the pyridone at $\mathrm{C}-1^{\prime}$ to form a furan ring. Thus, the planar structure of compound 5 was identified. The absolute configuration of C-6 was first determined as $S$ by referring to it potential biosynthetic precursor tolypyridone $\mathrm{C}(3) .{ }^{13} \mathrm{C}$ NMR calculation using the GIAO method at MPW1PW91/6-311+G(2d,p) levels in Guassian09 was conducted for four potential isomers of this compound (and the following three compounds), C1 $(3 R, 4 S, 6 S), \mathbf{C 2}(3 S, 4 R, 6 S), \mathbf{C 3}(3 S, 4 S, 6 S)$ and $\mathbf{C 4}(3 R, 4 R, 6 S)$ (Fig. S1). One-to-one linear correlation between $\mathbf{5}$ and C1-C4 and DP4+ probability calculation showed that calculated data of both $\mathbf{C 1}$ and $\mathbf{C 2}$ fitted well with $\mathbf{5}$ and compound 7 (see 2.1.5) (Fig. S8, Table S17-S19). This result indicated that relative configuration of 5 was $3 R^{*}, 4 S^{*}, 6 S$, but its absolute configuration was not distinguished by ${ }^{13} \mathrm{C}$ NMR calculation. Then calculated ECD showed that the experimental CD spectrum of 5 was close to the calculated spectrum of $3 S, 4 R, 6 S$ isomer (Fig. S20A). In summary, the absolute configuration of this compound was identified as $3 S, 4 R, 6 S$.

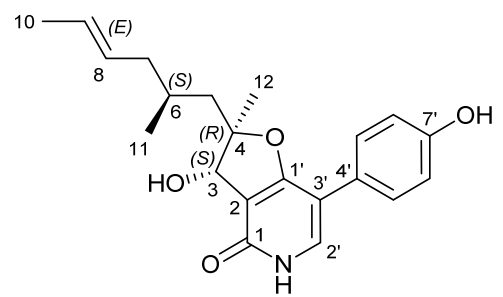

5

Table S8. NMR data of compound 5 (in DMSO- $d_{6}, 600 \mathrm{MHz}$ for ${ }^{1} \mathrm{H}$ and $150 \mathrm{MHz}$ for ${ }^{13} \mathrm{C}, J$ in $\mathrm{Hz}$ )

\begin{tabular}{|c|c|c|c|c|}
\hline Pos. & $\delta_{\mathrm{H}}$ & $\delta_{\mathrm{C}}$ & ${ }^{1} \mathrm{H}-{ }^{1} \mathrm{H}$ COSY & HMBC \\
\hline 1 & - & 160.5 & & \\
\hline 2 & - & 111.2 & & \\
\hline 3 & $4.58(\mathrm{~d}, 7.0)$ & 74.2 & $3-\mathrm{OH}$ & $1,2,3$ \\
\hline 4 & - & 94.3 & & \\
\hline 5 & $\begin{array}{l}1.61(\mathrm{dd}, 14.4,4.7) \\
\mathrm{a} \\
\begin{array}{c}1.28(\mathrm{dd}, 14.4,8.6) \\
\mathrm{b}\end{array}\end{array}$ & 44.2 & $\begin{array}{l}5 b \\
5 a\end{array}$ & $\begin{array}{l}3,4,6,7,11,12 \\
3,4,6,7,11,12\end{array}$ \\
\hline 6 & $1.66(\mathrm{td}, 12.5,6.4)$ & 28.6 & $7 a, 11$ & $4,5,7,8,11$ \\
\hline 7 & $\begin{array}{l}1.89, \mathrm{a} \\
1.78, \mathrm{~b}\end{array}$ & 40.7 & $\begin{array}{c}6,7 b, 8 \\
7 a, 8\end{array}$ & $6,8,9,11$ \\
\hline 8 & 5.25 & 129.3 & $7 \mathrm{a}, 7 \mathrm{~b}, 9$ & $6,7,9,10$ \\
\hline 9 & 5.25 & 126.1 & 8,10 & $7,8,10$ \\
\hline
\end{tabular}




\begin{tabular}{ccccc}
10 & $1.52(\mathrm{~d}, 3.6)$ & 17.7 & 9 & 8,9 \\
11 & $0.88(\mathrm{~d}, 6.6)$ & 21.5 & 6 & $5,6,7$ \\
12 & $1.37(\mathrm{~s})$ & 18.9 & & $3,4,5$ \\
$1^{\prime}$ & - & 165.2 & & \\
$2^{\prime}$ & $7.39(\mathrm{br} \mathrm{s})$ & 134.3 & $=\mathrm{NH}$ & $1,2,1^{\prime}, 3^{\prime}, 4^{\prime}$ \\
$3^{\prime}$ & - & 108.4 & & \\
$4^{\prime}$ & - & 123.9 & & \\
$5^{\prime} / 9^{\prime}$ & $7.33(\mathrm{~d}, 8.7)$ & 128.3 & $6^{\prime}, 8^{\prime}$ & $3^{\prime}, 6^{\prime}, 7^{\prime}, 8^{\prime}$ \\
$6^{\prime} / 8^{\prime}$ & $6.76(\mathrm{~d}, 8.7)$ & 115.2 & $5^{\prime}, 9^{\prime}$ & $4^{\prime}, 6^{\prime}, 7^{\prime}, 8^{\prime}$ \\
$7^{\prime}$ & - & 156.4 & & \\
$7^{\prime}-\mathrm{OH}$ & $9.45($ br s $)$ & & & $6^{\prime}, 7^{\prime}, 8^{\prime}$ \\
$=\mathrm{NH}$ & $11.26($ br s $)$ & & $2^{\prime}$ & \\
$3-\mathrm{OH}$ & $5.22(\mathrm{~d}, 7.0)$ & & 3 & $2,3,4^{\prime}$ \\
\hline
\end{tabular}

\subsubsection{Compound 6 tolypyridone $F$}

Tolypyridone F (6) was isolated as light yellow amorphous powder (methanol), quasi-molecular ion given by HR-ESI-MS was $m / z 356.1867[\mathrm{M}+\mathrm{H}]^{+}$, indicative of molecular formula $\mathrm{C}_{21} \mathrm{H}_{25} \mathrm{NO}_{4}\left([\mathrm{M}+\mathrm{H}]^{+}\right.$ calcd for $\left.\mathrm{C}_{21} \mathrm{H}_{26} \mathrm{NO}_{4}, 356.1862\right) .{ }^{1} \mathrm{H}$ NMR and ${ }^{13} \mathrm{C}$ NMR showed three methyl groups $\left(\delta_{\mathrm{H}} 0.93, \delta_{\mathrm{C}} 21.1, \mathrm{CH}_{3}-\right.$ $\left.11 ; \delta_{\mathrm{H}} 1.20, \delta_{\mathrm{C}} 22.6, \mathrm{CH}_{3}-12 ; \delta_{\mathrm{H}} 1.62, \delta_{\mathrm{C}} 17.8, \mathrm{CH}_{3}-10\right)$, two methenes $\left(\delta_{\mathrm{H}} 1.83\right.$ and $1.59, \delta_{\mathrm{C}} 38.9, \mathrm{CH}_{2}-5 ; \delta_{\mathrm{H}}$ 2.19 and $\left.1.87, \delta_{\mathrm{C}} 40.1, \mathrm{CH}_{2}-7\right)$, two $\mathrm{sp}^{3}$ methine $\left(\delta_{\mathrm{H}} 4.52, \delta_{\mathrm{C}} 75.1, \mathrm{CH}-3 ; \delta_{\mathrm{H}} 1.80, \delta_{\mathrm{C}} 28.7, \mathrm{CH}-6\right)$, three exchangeable protons $\left(\delta_{\mathrm{H}} 5.17,3-\mathrm{OH} ; \delta_{\mathrm{H}} 9.45,7^{\prime}-\mathrm{OH} ; \delta_{\mathrm{H}} 11.25,=\mathrm{NH}\right)$, one $\mathrm{sp}^{3}$ quaternary carbon $\left(\delta_{\mathrm{C}} 93.5\right.$, C-4), two olefinic carbons $(\delta \mathrm{H} 5.43, \delta \mathrm{C} 129.7, \mathrm{CH}-8 ; \delta \mathrm{H} 5.39, \delta \mathrm{C} 125.6, \mathrm{CH}-9)$, a carboxyl signal $(\delta \mathrm{C} 165.1, \mathrm{C}-$ $1)$, and ten other $\mathrm{sp}^{2}$ carbon and related proton signals $\left(\delta_{\mathrm{C}} 160.5, \mathrm{C}-1^{\prime} ; \delta_{\mathrm{H}} 7.38, \delta_{\mathrm{C}} 134.4, \mathrm{CH}-2^{\prime} ; \delta_{\mathrm{C}} 108.5, \mathrm{C}-\right.$ $\left.3^{\prime} ; \delta_{\mathrm{C}} 123.9, \mathrm{C}-4^{\prime} ; \delta_{\mathrm{H}} 7.32, \delta_{\mathrm{C}} 128.2, \mathrm{CH}-5^{\prime}, 9^{\prime} ; \delta_{\mathrm{H}} 6.74, \delta_{\mathrm{C}} 115.1, \mathrm{CH}-6^{\prime}, 8^{\prime} ; \delta_{\mathrm{C}} 156.4, \mathrm{C}-7^{\prime}\right)$. All data were assigned unambiguously according to HSQC and HMBC spectra. The HMBC correlation patterns of this compound resembled those of tolypyridone E (5). Based on MS and NMR, the planar structure of this compound was established, and it was a diastereomer of tolypyridone E (5). C-6 configuration of 6 was determined as $S$ by biosynthetic consideration. Calculated ${ }^{13} \mathrm{C}$ NMR data of $\mathbf{C} \mathbf{3}$ and $\mathbf{C 4}$ fitted with compounds 6 and 8 (see 2.1.6), so relative configuration of 6 was assigned as $3 R^{*}, 4 R^{*}, 6 S$ (Fig. S8, Table S17-S19). Then by comparing experimental and calculated CD spectra, the absolute configuration was identified as $3 R, 4 R$, $6 S$ (Fig. S20B). This compound was named as tolypyridone F (6). 


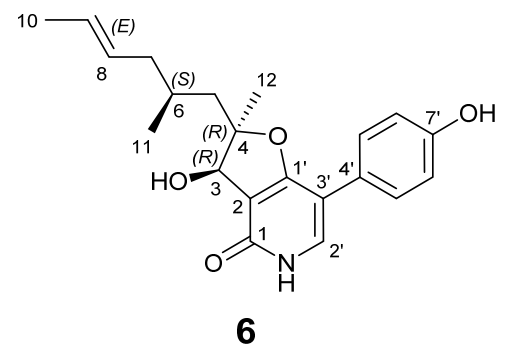

Table S9. NMR data of compound 6 (in DMSO- $d_{6}, 600 \mathrm{MHz}$ for ${ }^{1} \mathrm{H}$ and $150 \mathrm{MHz}$ for ${ }^{13} \mathrm{C}, J$ in $\mathrm{Hz}$ )

\begin{tabular}{|c|c|c|c|c|}
\hline Pos. & $\delta_{\mathrm{H}}$ & $\delta \mathrm{C}$ & ${ }^{1} \mathrm{H}-{ }^{1} \mathrm{H}$ COSY & HMBC \\
\hline 1 & - & 160.5 & & \\
\hline 2 & - & 111.2 & & \\
\hline 3 & $4.52(\mathrm{~d}, 7.3)$ & 75.1 & $3-\mathrm{OH}$ & $1,2,4,1^{\prime}, 12$ \\
\hline 4 & - & 93.5 & & \\
\hline \multirow{2}{*}{5} & $1.83, \mathrm{a}$ & \multirow{2}{*}{38.9} & $5 b$ & $3,4,6,7,11,12$ \\
\hline & $\begin{array}{c}1.59(\mathrm{dd}, 13.8,4.6) \\
\mathrm{b}\end{array}$ & & $5 \mathrm{a}, 6$ & $3,4,6,7,11,12$ \\
\hline 6 & 1.80 & 28.7 & $5 a, 7,11$ & $4,5,7,8,11$ \\
\hline \multirow{2}{*}{7} & $2.19, \mathrm{a}$ & \multirow{2}{*}{40.1} & $7 b, 8$ & $6,8,9,11$ \\
\hline & $1.87, \mathrm{~b}$ & & $7 \mathrm{a}, 8$ & $6,8,9,11$ \\
\hline 8 & 5.43 & 129.7 & 7,9 & $6,7,9,10$ \\
\hline 9 & 5.39 & 125.6 & 8,10 & $7,8,10$ \\
\hline 10 & $1.62(\mathrm{~d}, 5.9)$ & 17.8 & 9 & 8,9 \\
\hline 11 & $0.93(\mathrm{~d}, 6.4)$ & 21.1 & 6 & 5,6 \\
\hline 12 & $1.20(\mathrm{~s})$ & 22.6 & & $3,4,5$ \\
\hline $1^{\prime}$ & - & 165.1 & & \\
\hline $2^{\prime}$ & 7.38 (br s) & 134.4 & $=\mathrm{NH}$ & $1,1^{\prime}, 3^{\prime}, 4^{\prime}$ \\
\hline $3^{\prime}$ & - & 108.5 & & \\
\hline $4^{\prime}$ & - & 123.9 & & \\
\hline $5^{\prime} / 9^{\prime}$ & $7.32(\mathrm{~d}, 8.7)$ & 128.2 & $6^{\prime}, 8^{\prime}$ & $3^{\prime}, 5^{\prime}, 7^{\prime}, 9^{\prime}$ \\
\hline $6^{\prime} / 8^{\prime}$ & $6.74(\mathrm{~d}, 8.7)$ & 115.1 & $5^{\prime}, 9^{\prime}$ & $4^{\prime}, 7^{\prime}$ \\
\hline $7^{\prime}$ & - & 156.4 & & \\
\hline 7'-OH & 9.45 (br s) & & & $6^{\prime}, 7^{\prime}, 8^{\prime}$ \\
\hline$=\mathrm{NH}$ & 11.25 (br s) & & $2^{\prime}$ & \\
\hline $3-\mathrm{OH}$ & $5.17(\mathrm{~d}, 7.3)$ & & 3 & $2,3,4$ \\
\hline
\end{tabular}




\subsubsection{Compound 7 tolypyridone $G$}

Tolypyridone G (7) was isolated as light yellow amorphous powder, quasi-molecular ion given by HRESI-MS was $m / z 356.1861[\mathrm{M}+\mathrm{H}]^{+}$, indicative of molecular formula $\mathrm{C}_{21} \mathrm{H}_{25} \mathrm{NO}_{4}\left([\mathrm{M}+\mathrm{H}]^{+}\right.$calcd for $\left.\mathrm{C}_{21} \mathrm{H}_{26} \mathrm{NO}_{4}, 356.1862\right) .{ }^{1} \mathrm{H}$ NMR and ${ }^{13} \mathrm{C}$ NMR showed three methyl groups $\left(\delta_{\mathrm{H}} 0.82, \delta_{\mathrm{C}} 21.2, \mathrm{CH}_{3}-11 ; \delta_{\mathrm{H}}\right.$ $\left.1.35, \delta_{\mathrm{C}} 18.6, \mathrm{CH}_{3}-12 ; \delta_{\mathrm{H}} 1.60, \delta_{\mathrm{C}} 17.7, \mathrm{CH}_{3}-10\right)$, two methenes $\left(\delta_{\mathrm{H}} 1.53\right.$ and $1.38, \delta_{\mathrm{C}} 44.5, \mathrm{CH}_{2}-5 ; \delta_{\mathrm{H}} 1.89$ and $\left.1.81, \delta_{\mathrm{C}} 41.0, \mathrm{CH}_{2}-7\right)$, two $\mathrm{sp}^{3}$ methine $\left(\delta_{\mathrm{H}} 4.58, \delta_{\mathrm{C}} 74.4, \mathrm{CH}-3 ; \delta_{\mathrm{H}} 1.66, \delta_{\mathrm{C}} 28.7, \mathrm{CH}-6\right)$, three exchangeable protons $\left(\delta_{\mathrm{H}} 5.22,3-\mathrm{OH} ; \delta_{\mathrm{H}} 9.44,7^{\prime}-\mathrm{OH} ; \delta_{\mathrm{H}} 11.25,=\mathrm{NH}\right)$, one sp ${ }^{3}$ quaternary carbon $\left(\delta_{\mathrm{C}} 94.3\right.$, $\mathrm{C}-4)$, two olefinic carbons $\left(\delta_{\mathrm{H}} 5.35, \delta_{\mathrm{C}} 129.4, \mathrm{CH}-8 ; \delta_{\mathrm{H}} 5.35, \delta_{\mathrm{C}} 126.2, \mathrm{CH}-9\right)$, a carboxyl signal $\left(\delta_{\mathrm{C}} 165.2, \mathrm{C}-\right.$ $\left.1^{\prime}\right)$, and ten other $\mathrm{sp}^{2}$ carbon and related proton signals $\left(\delta_{\mathrm{C}} 160.5, \mathrm{C}-1 ; \delta_{\mathrm{H}} 7.37, \delta_{\mathrm{C}} 134.4, \mathrm{CH}-2^{\prime} ; \delta_{\mathrm{C}} 108.4, \mathrm{C}-\right.$ 3'; $\left.\delta_{\mathrm{C}} 123.9, \mathrm{C}-4^{\prime} ; \delta_{\mathrm{H}} 7.32, \delta_{\mathrm{C}} 128.3, \mathrm{CH}-5^{\prime}, 9^{\prime} ; \delta_{\mathrm{H}} 6.75, \delta_{\mathrm{C}} 115.2, \mathrm{CH}-6^{\prime}, 8^{\prime} ; \delta_{\mathrm{C}} 156.4, \mathrm{C}^{\prime} 7^{\prime}\right)$. All data were assigned unambiguously according to HSQC and HMBC spectra. The HMBC correlation patterns of this compound highly resembled those of tolypyridone E (5) and tolypyridone F (6). Based on MS and NMR, this compound was also a diastereomer of the former two compounds (5 and $\mathbf{6})$, and it was named as tolypyridone $\mathrm{G}$ (7). Same as $\mathbf{5}$, relative configuration was assigned as $3 S^{*}, 4 R^{*}$ by ${ }^{13} \mathrm{C}$ NMR calculation by Guassian09 software using the GIAO method at MPW1PW91/6-311+G(2d,p) levels (Fig. S8, Table S17-S19). Absolute configuration was identified as $3 R, 4 S, 6 S$, as the experimental CD spectrum of 7 was similar to $3 S, 4 R, 6 S$ isomer in ECD calculation (Fig. S20A).

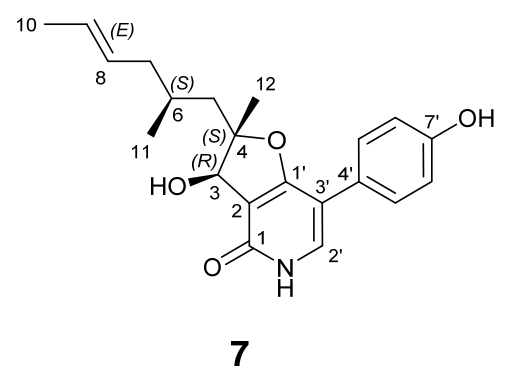

Table S10. NMR data of compound 7 (in DMSO- $d_{6}, 600 \mathrm{MHz}$ for ${ }^{1} \mathrm{H}$ and $150 \mathrm{MHz}$ for ${ }^{13} \mathrm{C}, J$ in $\mathrm{Hz}$ )

\begin{tabular}{ccccc}
\hline Pos. & $\delta_{\mathrm{H}}$ & $\delta_{\mathrm{C}}$ & ${ }^{1} \mathrm{H}-{ }^{1} \mathrm{H}$ COSY & HMBC \\
\hline 1 & - & 160.5 & & \\
2 & - & 111.0 & & \\
3 & $4.58(\mathrm{~d}, 7.1)$ & 74.4 & $3-\mathrm{OH}$ & $1,2,3$ \\
4 & - & 94.3 & & \\
& $1.53(\mathrm{dd}, 14.4,3.6)$, & & $5 \mathrm{~b}$ & $3,4,6,7,11,12$ \\
5 & $\mathrm{a}$ & 44.5 & $5 \mathrm{a}, 6$ & $3,4,6,7,11,12$ \\
& $1.38, \mathrm{~b}$ & & & $4,5,7,8,11$ \\
6 & 1.66 & 28.7 & $5 \mathrm{~b}, 7 \mathrm{~b}, 11$ & $4,6,8,9,11$ \\
7 & $1.89, \mathrm{a}$ & 41.0 & $7 \mathrm{~b}, 8$ & 5,12 \\
& & & &
\end{tabular}




\begin{tabular}{ccccc} 
& $1.81, \mathrm{~b}$ & & $6,7 \mathrm{a}, 8$ & $5,6,8,9,11$ \\
8 & 5.35 & 129.4 & $7 \mathrm{a}, 7 \mathrm{~b}$ & 7,10 \\
9 & 5.35 & 126.2 & 10 & 7,10 \\
10 & $1.60(\mathrm{~d}, 3.6)$ & 17.7 & 9 & 8,9 \\
11 & $0.82(\mathrm{~d}, 6.6)$ & 21.2 & 6 & $5,6,7$ \\
12 & $1.35(\mathrm{~s})$ & 18.6 & & $3,4,5$ \\
$1^{\prime}$ & - & 165.2 & & \\
$2^{\prime}$ & $7.37(\mathrm{br} \mathrm{s})$ & 134.4 & $=\mathrm{NH}$ & $1,2,1^{\prime}, 3^{\prime}, 4^{\prime}$ \\
$3^{\prime}$ & - & 108.4 & & \\
$4^{\prime}$ & - & 123.9 & & $3^{\prime}, 6^{\prime}, 7^{\prime}, 8^{\prime}$ \\
$5^{\prime} / 9^{\prime}$ & $7.32(\mathrm{~d}, 8.6)$ & 128.3 & $6^{\prime}, 8^{\prime}$ & $4^{\prime}, 7^{\prime}$ \\
$6^{\prime} / 8^{\prime}$ & $6.75(\mathrm{~d}, 8.6)$ & 115.2 & $5^{\prime}, 9^{\prime}$ & \\
$7^{\prime}$ & - & 156.4 & & $6^{\prime}, 7^{\prime}, 8^{\prime}$ \\
$7^{\prime}-\mathrm{OH}$ & $9.44(\mathrm{br} \mathrm{s})$ & & & \\
\hline $\mathrm{NH}$ & $11.25(\mathrm{br} \mathrm{s})$ & & $2,3,2^{\prime}$ & \\
$3-\mathrm{OH}$ & $5.22(\mathrm{~d}, 7.0)$ & & 3 & \\
\hline
\end{tabular}

\subsubsection{Compound 8 tolypyridone $\mathrm{H}$}

Tolypyridone H (8) was isolated as light yellow amorphous powder, quasi-molecular ion given by HRESI-MS was $m / z 356.1861[\mathrm{M}+\mathrm{H}]^{+}$, indicative of molecular formula $\mathrm{C}_{21} \mathrm{H}_{25} \mathrm{NO}_{4}\left([\mathrm{M}+\mathrm{H}]^{+}\right.$calcd for $\left.\mathrm{C}_{21} \mathrm{H}_{26} \mathrm{NO}_{4}, 356.1862\right) .{ }^{1} \mathrm{H}$ NMR and ${ }^{13} \mathrm{C}$ NMR showed three methyl groups $\left(\delta_{\mathrm{H}} 0.98, \delta \mathrm{C} 20.4, \mathrm{CH}_{3}-11 ; \delta_{\mathrm{H}}\right.$ $\left.1.20, \delta_{\mathrm{C}} 22.4, \mathrm{CH}_{3}-12 ; \delta_{\mathrm{H}} 1.65, \delta_{\mathrm{C}} 17.8, \mathrm{CH}_{3}-10\right)$, two methenes $\left(\delta_{\mathrm{H}} 1.77\right.$ and $1.63, \delta_{\mathrm{C}} 39.1, \mathrm{CH}_{2}-5 ; \delta_{\mathrm{H}} 2.01$ and 1.91, $\left.\delta_{\mathrm{C}} 41.4, \mathrm{CH}_{2}-7\right)$, two methine ( $\left.\delta_{\mathrm{H}} 4.53, \delta_{\mathrm{C}} 75.5, \mathrm{CH}-3 ; \delta_{\mathrm{H}} 1.81, \delta_{\mathrm{C}} 28.9, \mathrm{CH}-6\right)$, three exchangeable protons $\left(\delta_{\mathrm{H}} 5.18,3-\mathrm{OH} ; \delta_{\mathrm{H}} 9.46,7^{\prime}-\mathrm{OH} ; \delta_{\mathrm{H}} 11.27,=\mathrm{NH}\right)$, one $\mathrm{sp}^{3}$ quaternary carbon $\left(\delta_{\mathrm{C}} 93.4, \mathrm{C}-4\right)$, two olefinic carbons $\left(\delta_{\mathrm{H}} 5.43, \delta_{\mathrm{C}} 129.8, \mathrm{CH}-8 ; \delta_{\mathrm{H}} 5.43, \delta_{\mathrm{C}} 125.9, \mathrm{CH}-9\right)$, a carboxyl signal $\left(\delta_{\mathrm{C}} 165.2, \mathrm{C}-1^{\prime}\right)$, and ten other $\mathrm{sp}^{2}$ signals including aromatic carbons and protons and enoyl carbon $\left(\delta_{\mathrm{C}} 160.5, \mathrm{C}-1 ; \delta_{\mathrm{H}} 7.40, \delta_{\mathrm{C}} 134.5, \mathrm{CH}-\right.$ $\left.2^{\prime} ; \delta_{\mathrm{C}} 108.5, \mathrm{C}-3^{\prime} ; \delta_{\mathrm{C}} 123.9, \mathrm{C}-4^{\prime} ; \delta_{\mathrm{H}} 7.35, \delta_{\mathrm{C}} 128.2, \mathrm{CH}-5^{\prime}, 9^{\prime} ; \delta_{\mathrm{H}} 6.76, \delta_{\mathrm{C}} 115.1, \mathrm{CH}-6^{\prime}, 8^{\prime} ; \delta_{\mathrm{C}} 156.3, \mathrm{C}-7^{\prime}\right)$. All data were assigned unambiguously according to HSQC and HMBC spectra. The above data indicated that the planar structure of this compound is same as compounds 5-7. C-6 configuration was defined as $S$ by referring to their common biosynthetic precursor tolypyridone C (3). Same as compound $\mathbf{6}$, linear fitting between calculated and experimental ${ }^{13} \mathrm{C}$ NMR data indicated that relative configuration was $3 R^{*}, 4 R^{*}, 6 S$ (Fig. S8, Table S17-S19). At last, the absolute configuration of this compound was identified as $3 S, 4 S, 6 S$ by ECD calculation (Fig. S20B). 


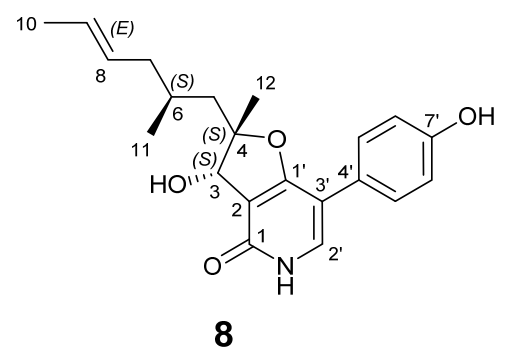

Table S11. NMR data of compound 8 (in DMSO- $d_{6}, 600 \mathrm{MHz}$ for ${ }^{1} \mathrm{H}$ and $150 \mathrm{MHz}$ for ${ }^{13} \mathrm{C}, J$ in $\mathrm{Hz}$ )

\begin{tabular}{|c|c|c|c|c|}
\hline Pos. & $\delta_{\mathrm{H}}$ & $\delta_{\mathrm{C}}$ & ${ }^{1} \mathrm{H}-{ }^{1} \mathrm{H}$ COSY & HMBC \\
\hline 1 & - & 160.5 & & \\
\hline 2 & - & 110.9 & & \\
\hline 3 & $4.53(\mathrm{~d}, 7.3)$ & 75.5 & $3-\mathrm{OH}$ & $2,3,4$ \\
\hline 4 & - & 93.4 & & \\
\hline \multirow{2}{*}{5} & $\begin{array}{c}1.77(\mathrm{dd}, 14.8,3.1) \\
\mathrm{a}\end{array}$ & \multirow{2}{*}{39.1} & $5 b$ & $4,6,7,11,12$ \\
\hline & $1.63, \mathrm{~b}$ & & $5 \mathrm{a}, 6$ & $3,4,6,7,11,12$ \\
\hline 6 & 1.81 & 28.9 & $5 b, 11$ & 7,11 \\
\hline \multirow{2}{*}{7} & $2.01, \mathrm{a}$ & \multirow{2}{*}{41.4} & $7 b, 8$ & $5,6,8,9,11$ \\
\hline & $1.91, \mathrm{~b}$ & & $7 a, 8$ & $5,6,8,9,11$ \\
\hline 8 & 5.43 & 129.8 & $7 \mathrm{a}, 7 \mathrm{~b}$ & $6,7,10$ \\
\hline 9 & 5.43 & 125.9 & 10 & 7,10 \\
\hline 10 & $1.65(\mathrm{~d}, 4.2)$ & 17.8 & 9 & 8,9 \\
\hline 11 & $0.98(\mathrm{~d}, 6.5)$ & 20.4 & 6 & $5,6,7$ \\
\hline 12 & $1.20(\mathrm{~s})$ & 22.4 & & $3,4,5$ \\
\hline $1^{\prime}$ & - & 165.2 & & \\
\hline $2^{\prime}$ & 7.40 (br s) & 134.5 & $=\mathrm{NH}$ & $1,1^{\prime}, 3^{\prime}, 4^{\prime}$ \\
\hline $3^{\prime}$ & - & 108.5 & & \\
\hline $4^{\prime}$ & - & 123.9 & & \\
\hline $5^{\prime} / 9^{\prime}$ & $7.35(\mathrm{~d}, 8.7)$ & 128.2 & $6^{\prime}, 8^{\prime}$ & $4^{\prime}, 6^{\prime}, 7^{\prime}, 8^{\prime}$ \\
\hline $6^{\prime} / 8^{\prime}$ & $6.76(\mathrm{~d}, 8.7)$ & 115.1 & $5^{\prime}, 9^{\prime}$ & $3^{\prime}, 5^{\prime}, 7^{\prime}, 9^{\prime}$ \\
\hline $7^{\prime}$ & - & 156.3 & & \\
\hline 7'-OH & 9.46 (br s) & & & $6^{\prime}, 7^{\prime}, 8^{\prime}$ \\
\hline$=\mathrm{NH}$ & 11.27 (br s) & & $2^{\prime}$ & \\
\hline $3-\mathrm{OH}$ & $5.18(\mathrm{~d}, 7.4)$ & & 3 & $2,3,4$ \\
\hline
\end{tabular}


Table S12. NMR data comparison of tolypoalbin (1) and F-14329 (2) with reported data (in $\mathrm{CDCl}_{3}, 600$ MHz for ${ }^{1} \mathrm{H}$ and $150 \mathrm{MHz}$ for ${ }^{13} \mathrm{C}, J$ is in $\mathrm{Hz}$ )

\begin{tabular}{|c|c|c|c|c|c|c|c|c|}
\hline \multirow[b]{2}{*}{ Pos. } & \multicolumn{2}{|c|}{ Tolypoalbin ${ }^{6 a}$} & \multicolumn{2}{|c|}{1} & \multicolumn{2}{|c|}{ F-143296a } & \multicolumn{2}{|c|}{2} \\
\hline & $\delta_{\mathrm{H}}$ & $\delta_{\mathrm{C}}$ & $\delta_{\mathrm{H}}$ & $\delta_{\mathrm{C}}$ & $\delta_{\mathrm{H}}$ & $\delta_{\mathrm{C}}$ & $\delta_{\mathrm{H}}$ & $\delta_{\mathrm{C}}$ \\
\hline 1 & 175.3 & & 175.4 & & 175.5 & & 175.4 & \\
\hline 2 & 100.5 & & 100.5 & & 100.4 & & 100.4 & \\
\hline 3 & 193.6 & & 193.6 & & 194.5 & & 194.7 & \\
\hline 4 & 34.0 & 3.80 & 34.0 & 3.79 (br s) & 34.3 & 3.70 & 34.4 & 3.72 \\
\hline 5 & 40.3 & $1.81,1.18$ & 40.3 & $1.81,1.17$ & 40.2 & $\begin{array}{c}1.75-1.85 \\
1.17\end{array}$ & 40.4 & $\begin{array}{c}1.75-1.85 \\
1.17\end{array}$ \\
\hline 6 & 31.3 & 1.38 & 31.3 & 1.37 & 31.3 & 1.36 & 31.4 & $1.36(\mathrm{~d}, 5.9)$ \\
\hline 7 & 40.4 & $1.98,1.81$ & 40.4 & $1.96,1.81$ & 40.3 & $\begin{array}{c}1.96,1.75- \\
1.85\end{array}$ & 40.4 & $\begin{array}{c}1.96,1.75- \\
1.85\end{array}$ \\
\hline 8 & 129.3 & 5.36 & 129.4 & 5.36 & 129.2 & 5.36 & 129.3 & 5.35 \\
\hline 9 & 126.4 & 5.41 & 126.4 & 5.41 & 126.6 & 5.41 & 126.6 & 5.42 \\
\hline 10 & 18.0 & $1.64(\mathrm{~d}, 5.5)$ & 18.0 & $1.64(\mathrm{~d}, 4.4)$ & 18.0 & $1.65(\mathrm{~d}, 6.0)$ & 18.1 & $1.64(\mathrm{~d}, 5.8)$ \\
\hline 11 & 19.5 & $0.87(\mathrm{~d}, 7.0)$ & 19.4 & $0.87(\mathrm{~d}, 5.9)$ & 19.5 & $0.86(\mathrm{~d}, 7.0)$ & 19.6 & $0.87(\mathrm{~d}, 6.5)$ \\
\hline 12 & 18.4 & $1.17(\mathrm{~d}, 6.5)$ & 18.0 & $1.17(\mathrm{~d}, 5.6)$ & 18.3 & $1.12(\mathrm{~d}, 7.0)$ & 18.4 & $1.15(\mathrm{~d}, 6.8)$ \\
\hline $1^{\prime}$ & 194.0 & & 194.1 & & 194.8 & & 194.9 & - \\
\hline $2^{\prime}$ & 37.4 & $\begin{array}{c}3.19(\mathrm{~d}, 14.0) \\
2.66(\mathrm{dd}, \\
14.0,8.0)\end{array}$ & 37.3 & $\begin{array}{c}3.17 \text { (br s) } \\
2.66\end{array}$ & 74.2 & $4.76(d, 7.0)$ & 74.3 & $4.74(\mathrm{~d}, 7.7)$ \\
\hline $3^{\prime}$ & 63.5 & $\begin{array}{c}3.99 \text { (br d, } \\
8.0)\end{array}$ & 63.5 & 3.99 (br s) & 65.7 & $3.98(\mathrm{~d}, 7.0)$ & 65.6 & $3.95(\mathrm{~d}, 7.8)$ \\
\hline $4^{\prime}$ & 130.3 & - & 130.4 & - & 130.0 & - & 130.0 & - \\
\hline $5^{\prime} / 9^{\prime}$ & 128.0 & 7.03 (br s) & 127.8 & 7.02 (br s) & 128.4 & $7.17(\mathrm{~d}, 7.3)$ & 128.5 & $7.17(\mathrm{~d}, 7.2)$ \\
\hline $6^{\prime} / 8^{\prime}$ & 115.8 & 6.77 (br s) & 115.5 & 6.76 (br s) & 115.6 & $6.70(\mathrm{~d}, 7.3)$ & 115.7 & $6.70(\mathrm{~d}, 7.2)$ \\
\hline $7^{\prime}$ & 155.0 & & 155.2 & - & 156.4 & - & 156.3 & - \\
\hline $7^{\prime} \mathrm{OH}$ & & 9.17 & & & & $9.17(\mathrm{~s})$ & & \\
\hline $2-\mathrm{OH}$ & & & & 6.05 (br s) & & 6.03 (br s) & & 5.56 (br s) \\
\hline
\end{tabular}




\subsection{Quantum chemistry calculation}

ECD and 13C NMR calculation methods The conformational distributions of truncated molecular models a $(3 S, 4 R, 6 S)$, b $(3 R, 4 R, 6 S)$, c $(3 R, 4 S, 6 S)$, d $(3 S, 4 S, 6 S)$ (Fig. S1) were calculated by CONFLEX version 7.0 using the MMFF94s force-field. Comformers for each coompound within the energy window of $3 \mathrm{kcal} / \mathrm{mol}$ were accepted, RMSD cutoff was $0.5 \AA$, maximum number of conformations was 100 . Then all accepted conformers were optimized with HF/6-31G(d) method in Gaussian09. ${ }^{7}$ Further optimization was conducted at B3LYP/6-31g(d) level to determine the dihedral angles. The optimized conformers were used for the ECD calculations, which were performed with Gaussian09 (B3LYP/TZVP). Solvent effects were taken into consideration by the polarizable-conductor calculation model (IEFPCM, methanol as the solvent). SpecDis software was used in the comparisons between experimental and calculated spectra, UV shift to the ECD spectra, Gaussian broadening of the excitations, and Boltzmann weighting of the spectra. Similarly, ${ }^{13} \mathrm{C}$ NMR chemical shifts were calculated by Gaussian09 (mPW1PW91/6-31+G(d,p)), solvent was deuterated dimethyl sulfoxide and solvent effects were evaluated by the IEFPCM solvent model, TMS was used as reference. Computed chemical shifts were scaled empirically according to $\delta_{\text {corr. }}=\delta_{\text {calcd. }} \times$ slope + intercept, where $\delta_{\text {calcd. }}$ is the calculated chemical shift, and slope and intercept are the slope and intercept resulting from

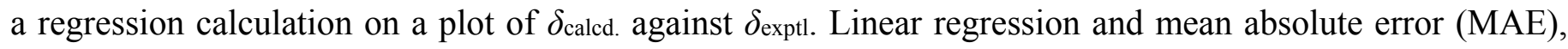
$\mathrm{DP} 4+$ probability test and $\mathrm{MAE}_{\Delta \Delta \delta}$ were applied in assignment between experimental and calculated data sets. ${ }^{8}$ Linearity correlations and MAE values are shown in Table S18, DP4+ probabilities are listed in Table S19, and MAE $\Delta \Delta \delta$ was listed in Table S20.

As shown by the results, linearity, MAE and DP4+ test failed to achieve one-to-one assignment between calculated and experimental data sets, so $\mathrm{MAE}_{\Delta \Delta \delta}$ value, a newly reported parameter for data assignment was employed. ${ }^{8 b}$ 
Fig. S1 Isomers for ECD and ${ }^{13} \mathrm{C}$ NMR calculation

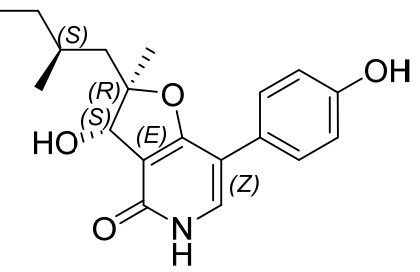

a

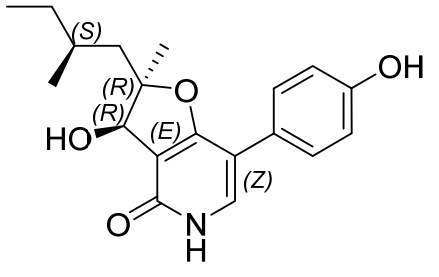

b<smiles>CCC(C)CC1(C)Oc2c(-c3ccc(O)cc3)c[nH]c(=O)c2C1O</smiles>

C

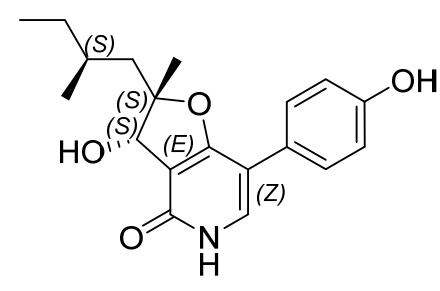

d

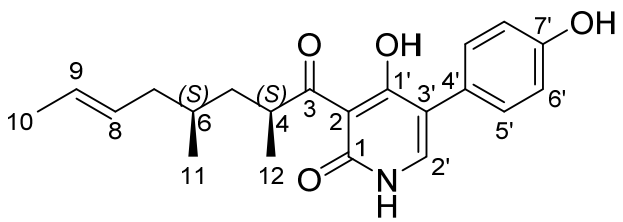

$3 a$

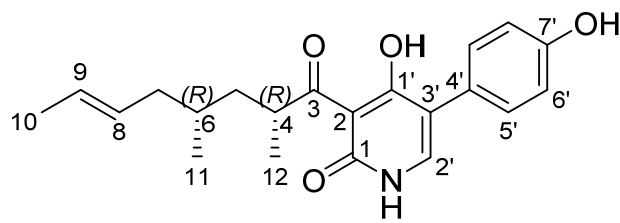

$3 a$

Table S13 Boltzmann distribution percentages of accepted conformers of a

\begin{tabular}{cc|cc|cc|cc}
\hline Conf. No. & Percentage & Conf. No. & Percentage & Conf. No. & Percentage & Conf. No. & Percentage \\
\hline a-c1 & 1.32 & a-c19 & 6.10 & a-c38 & 2.01 & a-c54 & 1.22 \\
a-c4 & 2.84 & a-c21 & 1.75 & a-c39 & 1.20 & a-c55 & 1.57 \\
a-c6 & 3.01 & a-c25 & 6.07 & a-c41 & 5.90 & a-c56 & 1.20 \\
a-c8 & 1.30 & a-c28 & 7.40 & a-c42 & 1.70 & a-c58 & 1.48 \\
a-c9 & 2.88 & a-c31 & 2.21 & a-c44 & 6.00 & a-c60 & 1.33 \\
a-c10 & 2.90 & a-c34 & 1.96 & a-c45 & 7.20 & a-c65 & 5.14 \\
a-c14 & 2.09 & a-c35 & 1.27 & a-c46 & 1.49 & a-c69 & 1.16 \\
a-c15 & 2.24 & a-c36 & 1.58 & a-c49 & 1.94 & a-c78 & 1.30 \\
a-c18 & 5.14 & a-c37 & 4.94 & a-c53 & 1.15 & & \\
\hline
\end{tabular}

Table S14 Boltzmann distribution percentages of accepted conformers of b

\begin{tabular}{cc|cc|cc|cc}
\hline Conf. No. & Percentage & Conf. No. & Percentage & Conf. No. & Percentage & Conf. No. & Percentage \\
\hline b-c1 & 2.41 & b-c13 & 2.15 & b-c23 & 2.14 & b-c36 & 2.12 \\
b-c2 & 2.37 & b-c14 & 5.75 & b-c26 & 1.88 & b-c38 & 2.46 \\
b-c3 & 1.30 & b-c15 & 5.00 & b-c27 & 2.76 & b-c39 & 2.76 \\
b-c4 & 4.32 & b-c17 & 2.28 & b-c29 & 2.43 & b-c43 & 2.64 \\
b-c6 & 1.25 & b-c18 & 1.89 & b-c30 & 1.65 & b-c46 & 1.60 \\
b-c8 & 4.96 & b-c19 & 2.98 & b-c31 & 2.82 & b-c48 & 2.81 \\
b-c9 & 4.27 & b-c20 & 2.55 & b-c32 & 2.33 & b-c52 & 2.77 \\
b-c11 & 3.09 & b-c21 & 5.66 & b-c33 & 2.81 & b-c55 & 4.06 \\
b-c12 & 3.10 & b-c22 & 2.20 & b-c34 & 2.44 & & \\
\hline
\end{tabular}

Table S15 Boltzmann distribution percentages of accepted conformers of c

\begin{tabular}{cc|cc|cc|cc}
\hline Conf. No. & Percentage & Conf. No. & Percentage & Conf. No. & Percentage & Conf. No. & Percentage \\
\hline c-c1 & 3.97 & c-c12 & 4.82 & c-c35 & 1.83 & c-c49 & 1.81 \\
c-c2 & 4.82 & c-c13 & 1.18 & c-c36 & 4.97 & c-c50 & 1.67 \\
c-c3 & 4.33 & c-c15 & 1.36 & c-c37 & 1.11 & c-c51 & 2.00 \\
\hline
\end{tabular}




\begin{tabular}{ll|ll|ll|ll}
\hline $\mathrm{c}-\mathrm{c} 4$ & 1.21 & $\mathrm{c}-\mathrm{c} 23$ & 2.45 & $\mathrm{c}-\mathrm{c} 38$ & 5.37 & $\mathrm{c}-\mathrm{c} 54$ & 1.11 \\
$\mathrm{c}-\mathrm{c} 5$ & 5.48 & $\mathrm{c}-\mathrm{c} 24$ & 1.05 & $\mathrm{c}-\mathrm{c} 39$ & 1.83 & $\mathrm{c}-\mathrm{c} 55$ & 1.60 \\
$\mathrm{c}-\mathrm{c} 6$ & 1.37 & $\mathrm{c}-\mathrm{c} 25$ & 5.05 & $\mathrm{c}-\mathrm{c} 40$ & 2.01 & $\mathrm{c}-\mathrm{c} 61$ & 1.46 \\
$\mathrm{c}-\mathrm{c} 7$ & 3.86 & $\mathrm{c}-\mathrm{c} 28$ & 2.43 & $\mathrm{c}-\mathrm{c} 43$ & 1.66 & $\mathrm{c}-\mathrm{c} 62$ & 1.25 \\
$\mathrm{c}-\mathrm{c} 9$ & 4.22 & $\mathrm{c}-\mathrm{c} 29$ & 5.44 & $\mathrm{c}-\mathrm{c} 44$ & 1.47 & & \\
$\mathrm{c}-\mathrm{c} 10$ & 4.74 & $\mathrm{c}-\mathrm{c} 30$ & 1.04 & $\mathrm{c}-\mathrm{c} 45$ & 1.27 & & \\
$\mathrm{c}-\mathrm{c} 11$ & 5.27 & $\mathrm{c}-\mathrm{c} 32$ & 1.67 & $\mathrm{c}-\mathrm{c} 47$ & 1.79 & & \\
\hline
\end{tabular}

Table S16 Boltzmann distribution percentages of accepted conformers of d

\begin{tabular}{cc|cc|cc|cc}
\hline Conf. No. & Percentage & Conf. No. & Percentage & Conf. No. & Percentage & Conf. No. & Percentage \\
\hline d-c1 & 3.34 & d-c11 & 4.11 & d-c23 & 1.06 & d-c56 & 6.86 \\
d-c2 & 3.78 & d-c13 & 3.68 & d-c24 & 1.80 & d-c61 & 8.02 \\
d-c3s1 & 3.44 & d-c14 & 1.08 & d-c26 & 1.20 & d-c64 & 6.13 \\
d-c4 & 1.05 & d-c15 & 1.01 & d-c27 & 1.39 & d-c65 & 2.13 \\
d-c5 & 1.08 & d-c16 & 3.19 & d-c30 & 1.05 & d-c68 & 7.21 \\
d-c6 & 4.16 & d-c20 & 3.12 & d-c31 & 1.37 & d-c76 & 1.83 \\
d-c7 & 3.43 & d-c21 & 1.26 & d-c33 & 1.78 & d-c77 & 6.60 \\
d-c10 & 3.16 & d-c22 & 1.38 & d-c34 & 1.35 & d-c81 & 7.94 \\
\hline
\end{tabular}

Table S17 Boltzmann distribution percentages of accepted conformers of $3 \mathrm{a}$ and $3 \mathrm{~b}$

\begin{tabular}{ccc}
\hline Isomers & Comformer & Percentage \\
\hline \multirow{3}{*}{ 3a } & 1 & 11.15 \\
& 2 & 49.78 \\
& 3 & 19.70 \\
3 & 4 & 19.37 \\
\hline \multirow{3}{*}{ 3b } & 1 & 7.11 \\
& 2 & 6.96 \\
& 3 & 71.05 \\
\hline
\end{tabular}


Fig. S2 Major conformers of a (top 12)

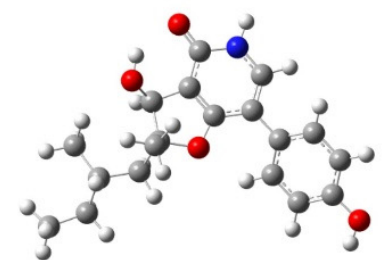

a-c28 (7.40\%)

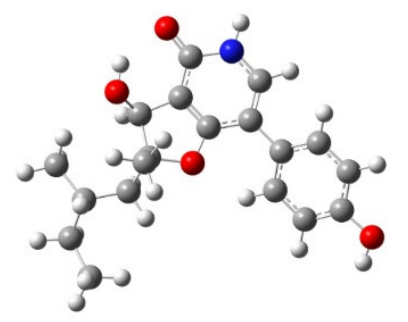

a-c25 (6.07\%)

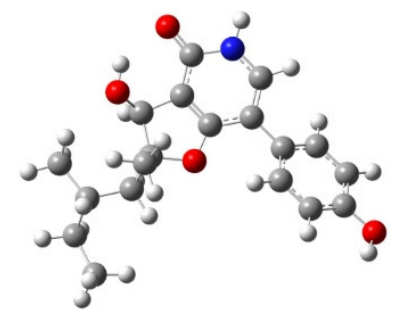

a-c18 (5.14\%)

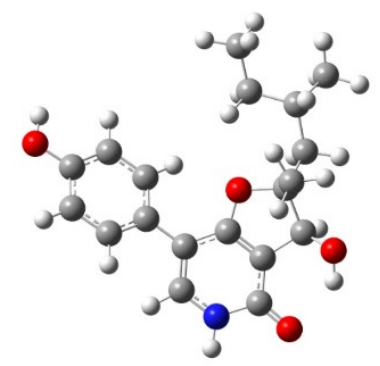

a-c6 (3.01\%)

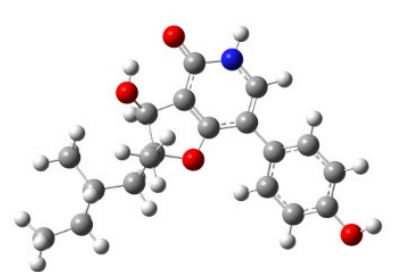

a-c45 (7.20\%)

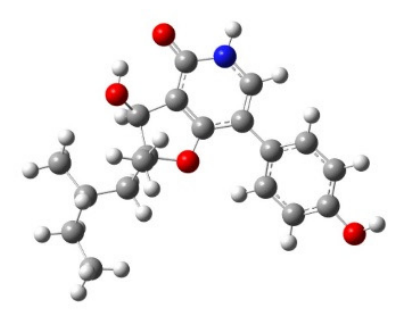

a-c44 (6.00\%)

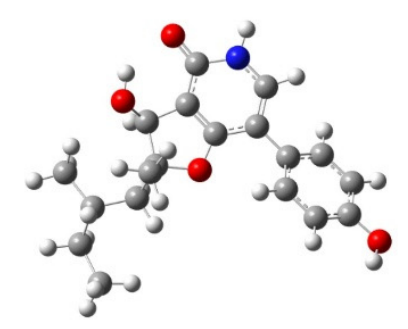

a-c65 (5.14\%)

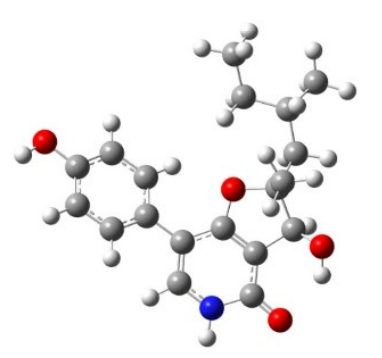

a-c10 (2.90\%)

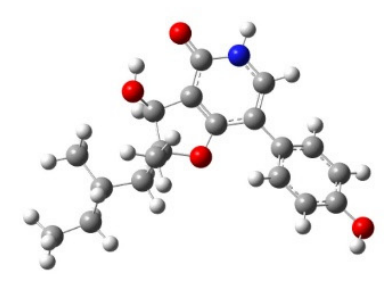

a-c19 (6.10\%)

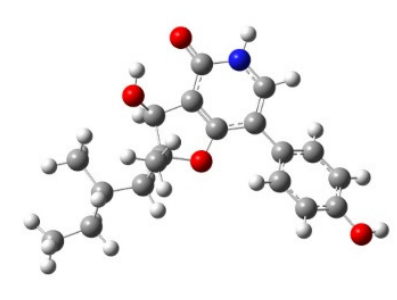

a-c41 (5.90\%)

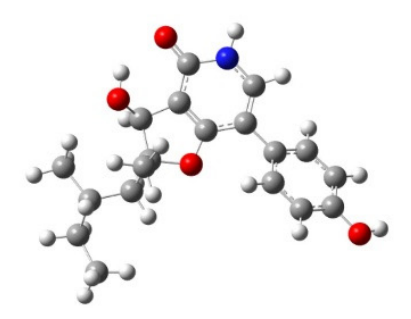

a-c37 (4.94\%)

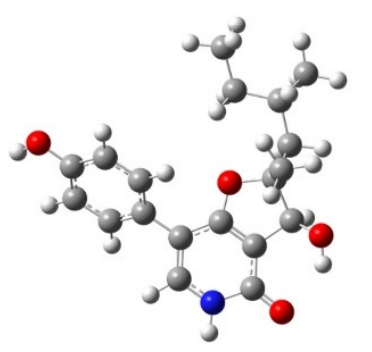

a-c9 $(2.88 \%)$ 
Fig. S3 Major conformers of b (top 12)

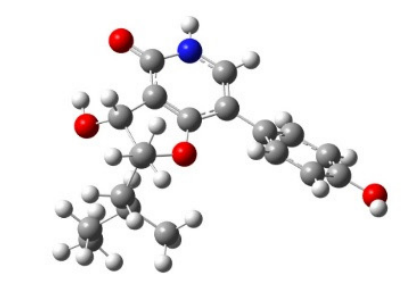

b-c14 (5.75\%)

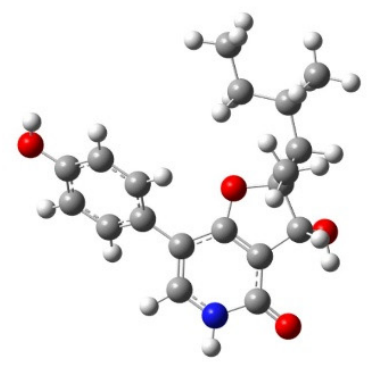

b-c8 $(4.96 \%)$

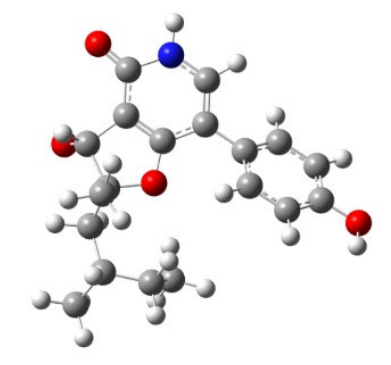

b-c55 (4.06\%)

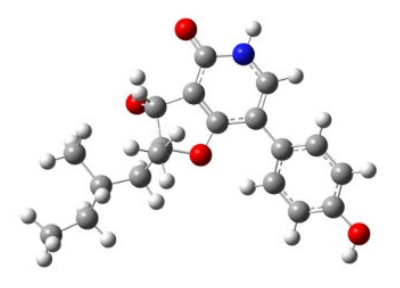

b-c19 (2.98\%)

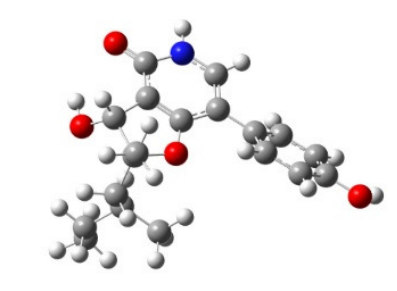

b-c21 (5.66\%)

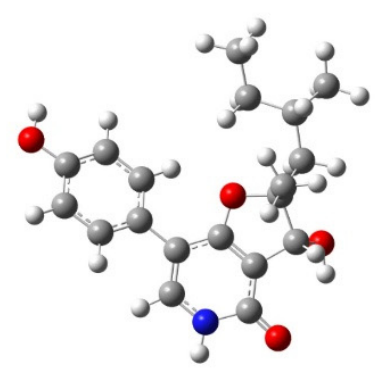

b-c4 (4.32\%)

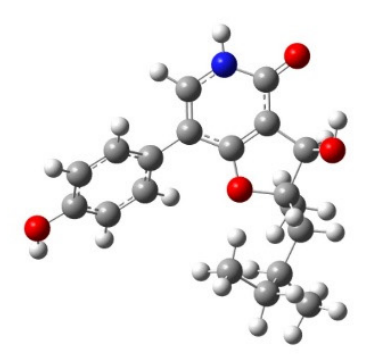

b-c12 (3.10\%)

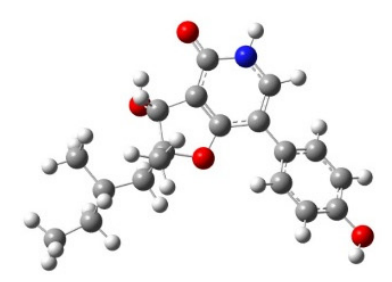

b-c31 (2.82\%)
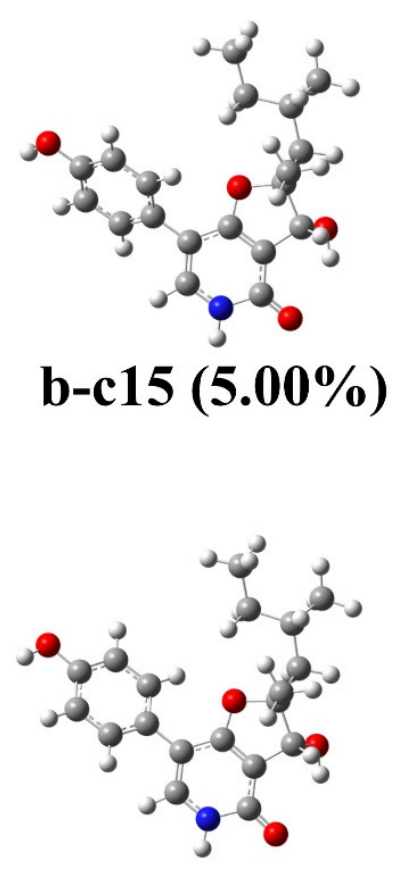

b-c9 $(4.27 \%)$

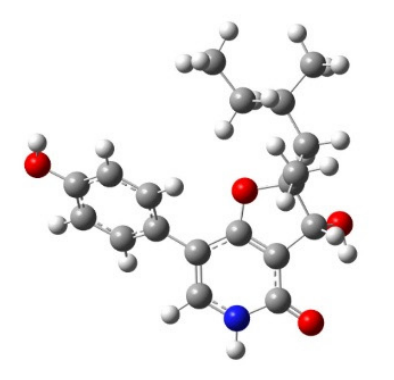

b-c11 (3.09\%)

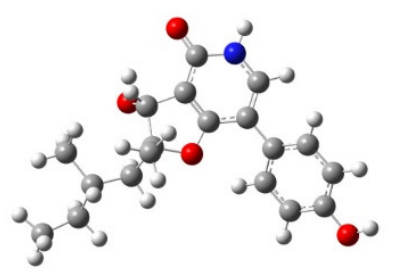

b-c33 (2.81\%) 
Fig. S4 Major conformers of c (top 12)

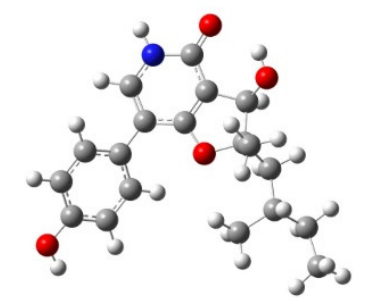

c-c5 (5.48\%)

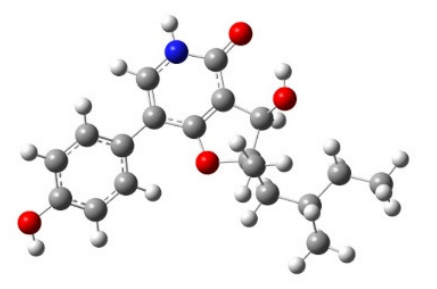

c-c29 (5.44\%)

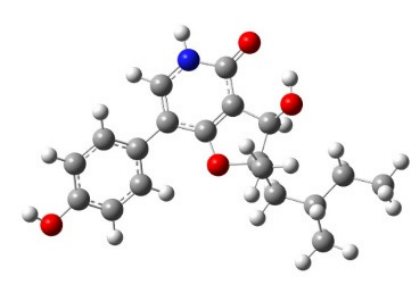

c-c38 (5.37\%)

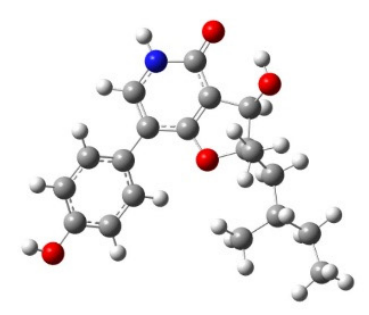

c-c11 (5.27\%)

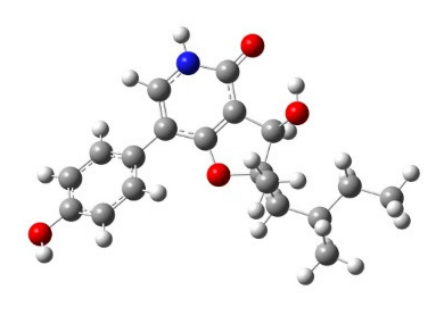

c-c25 (5.05\%)

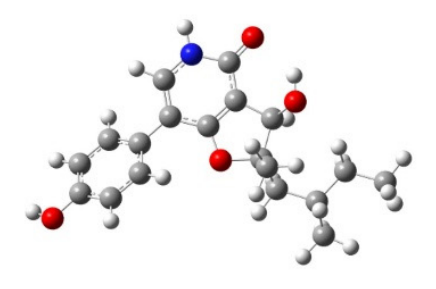

c-c36 (4.97\%)

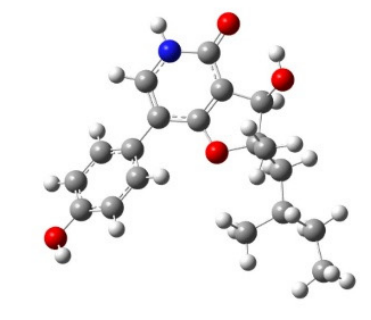

c-c2 $(4.82 \%)$

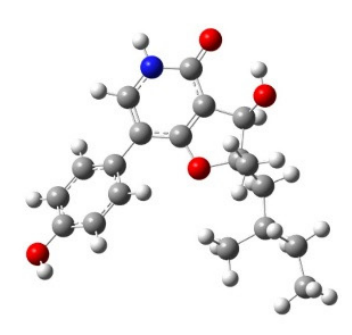

c-c12 (4.82\%)

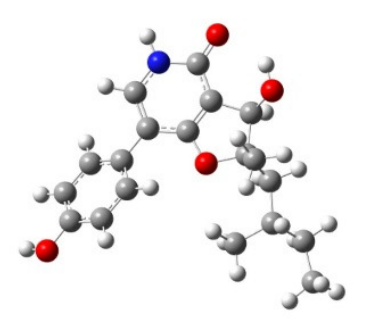

c-c10 $(4.74 \%)$

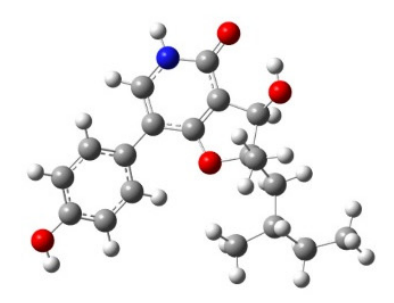

c-c3 $(4.33 \%)$

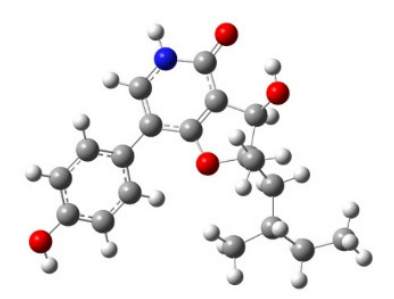

c-c9 $(4.22 \%)$

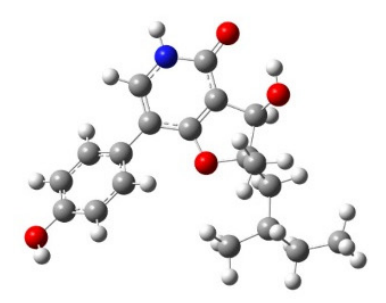

c-c1 $(3.97 \%)$ 
Fig. S5 Major conformers of d (top 12)

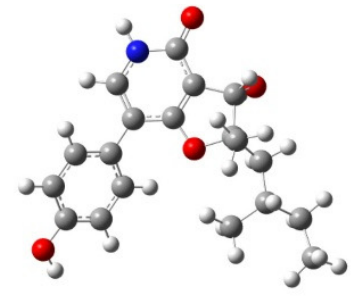

d-c61 (8.02\%)

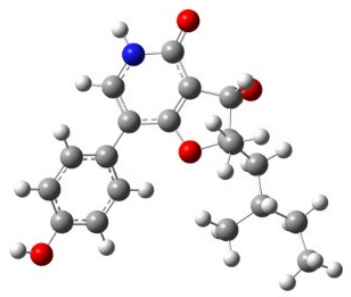

d-c81 (7.94\%)

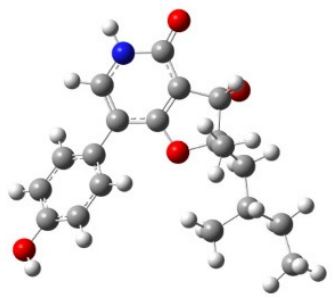

d-c68 (7.21\%)

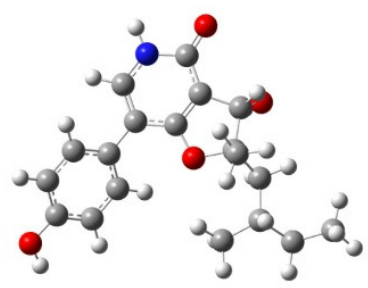

d-c56 (6.86\%)

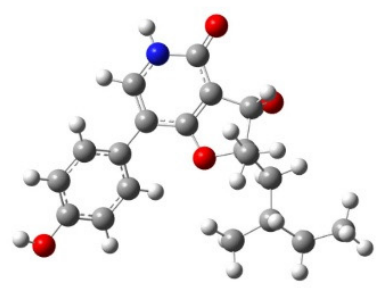

d-c77 (6.60\%)

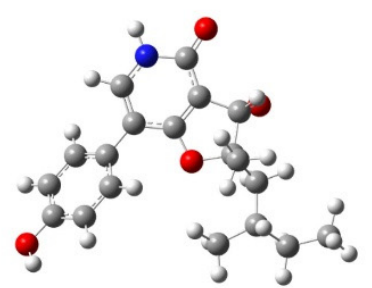

d-c64 (6.13\%)

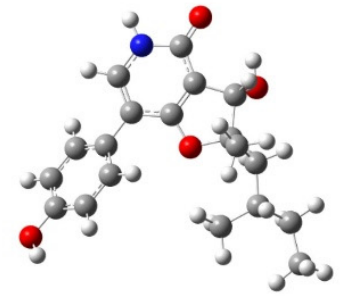

d-c6 (4.16\%)

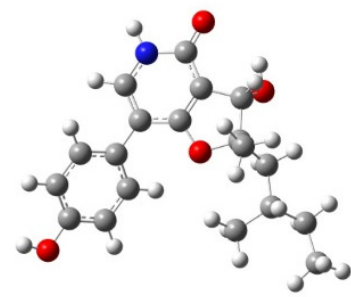

d-c13 (3.68\%)

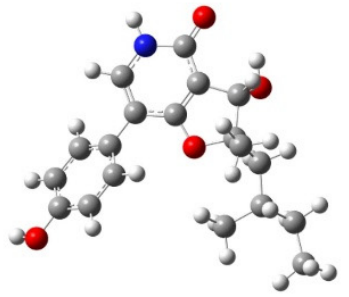

d-c11 (4.11\%)

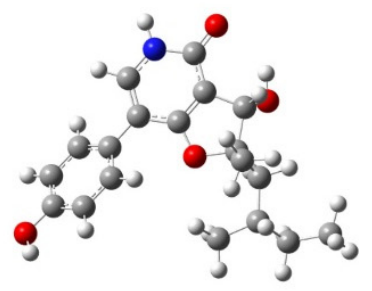

d-c3 (3.44\%)

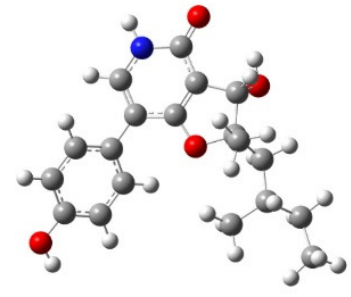

d-c2 $(3.78 \%)$ d-c7 $(3.43 \%)$ 
Fig. S6 Lowest energy conformers of $3 a$ and $3 b$

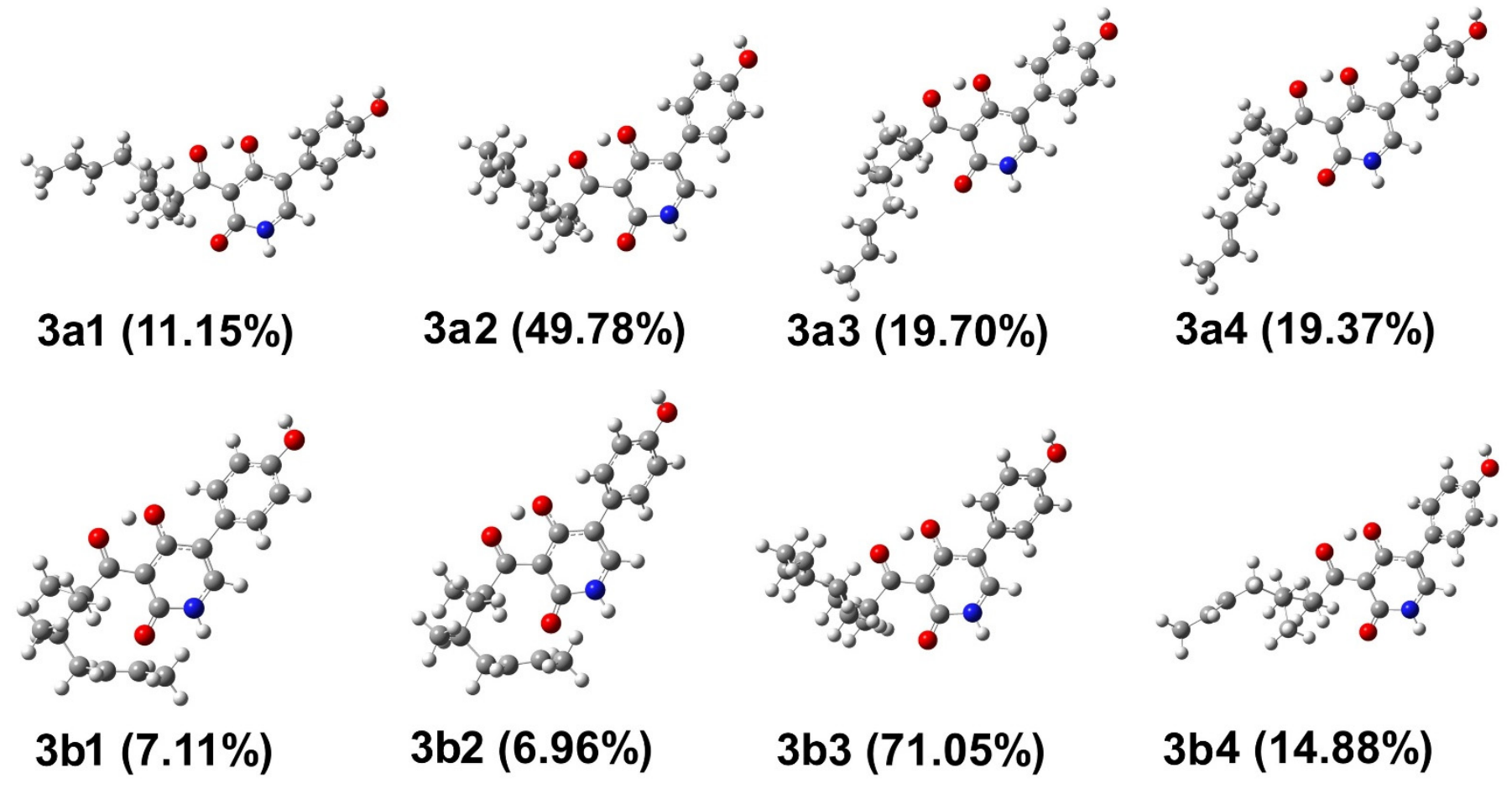


Table S18 Experimental ${ }^{13} \mathrm{C}$ NMR data of 5-8 and calculated ${ }^{13} \mathrm{C}$ NMR data of a-d

\begin{tabular}{|c|c|c|c|c|c|c|c|c|}
\hline No. & 5 & 6 & 7 & 8 & $\mathbf{a}$ & b & c & d \\
\hline Pos. & Experimental & Experimental & Experimental & Experimental & Calculated & Calculated & Calculated & Calculated \\
\hline C-1 & 160.5 & 160.5 & 160.5 & 160.5 & 157.7103 & 157.5717 & 157.7545 & 157.3990 \\
\hline $\mathrm{C}-2$ & 111.2 & 111.2 & 111 & 110.9 & 111.5291 & 112.0937 & 111.1369 & 111.2159 \\
\hline $\mathrm{C}-3$ & 74.2 & 75.1 & 74.4 & 75.5 & 80.7375 & 80.8976 & 81.7436 & 81.8389 \\
\hline $\mathrm{C}-4$ & 94.3 & 93.5 & 94.3 & 93.4 & 101.0103 & 99.0493 & 100.7183 & 98.4364 \\
\hline C-5 & 44.2 & 38.9 & 44.5 & 39.1 & 48.6802 & 42.0380 & 49.5598 & 41.9592 \\
\hline C-6 & 28.6 & 28.7 & 28.7 & 28.9 & 36.1465 & 35.0997 & 35.5856 & 35.3138 \\
\hline C-11 & 21.5 & 21.1 & 21.2 & 20.4 & 21.2994 & 22.3854 & 22.0557 & 21.7219 \\
\hline C-12 & 18.9 & 22.6 & 18.6 & 22.4 & 21.0677 & 26.2356 & 20.6625 & 24.8677 \\
\hline $\mathrm{C}-1^{\prime}$ & 165.2 & 165.1 & 165.2 & 165.2 & 162.2825 & 163.9043 & 162.5370 & 164.7746 \\
\hline C-2' & 134.3 & 134.4 & 134.4 & 134.5 & 135.2100 & 135.6944 & 135.2957 & 136.1197 \\
\hline C-3' & 108.4 & 108.5 & 108.4 & 108.5 & 112.3965 & 112.1164 & 112.5243 & 111.9974 \\
\hline C-4' & 123.9 & 123.9 & 123.9 & 123.9 & 125.6493 & 125.8311 & 125.6481 & 125.8370 \\
\hline $\mathrm{C}-5^{\prime}$ & 128.3 & 128.2 & 128.3 & 128.2 & 128.7747 & 128.8377 & 129.0665 & 129.6827 \\
\hline C-6' & 115.2 & 115.1 & 115.2 & 115.1 & 113.2978 & 113.8647 & 113.4532 & 113.3537 \\
\hline C-7' & 156.4 & 156.4 & 156.4 & 156.3 & 154.7484 & 154.7409 & 154.7834 & 154.7416 \\
\hline C-8' & 115.2 & 115.1 & 115.2 & 115.1 & 114.0196 & 113.3980 & 113.9011 & 113.9221 \\
\hline C-9' & 128.3 & 128.2 & 128.3 & 128.2 & 128.4215 & 128.6688 & 128.4181 & 128.1858 \\
\hline
\end{tabular}

Table S19 R square $\left(R^{2}\right)$ of the linear correlations and mean absolute error (MAE) values

\begin{tabular}{ccccccccc}
\hline \multirow{2}{*}{ No. } & \multicolumn{2}{c}{$\mathbf{5}$} & \multicolumn{2}{c}{$\mathbf{6}$} & \multicolumn{2}{c}{$\mathbf{7}$} & \multicolumn{2}{c}{$\mathbf{8}$} \\
\cline { 2 - 9 } & $\mathrm{R}^{2}$ & $\mathrm{MAE}$ & $\mathrm{R}^{2}$ & $\mathrm{MAE}$ & $\mathrm{R}^{2}$ & $\mathrm{MAE}$ & $\mathrm{R}^{2}$ & $\mathrm{MAE}$ \\
\hline 3R, 4S, 6S & 0.9967 & 2.736 & 0.9946 & 3.052 & 0.9969 & 2.734 & 0.9950 & 3.045 \\
3S, 4R, 6S & $\mathbf{0 . 9 9 6 7}$ & $\mathbf{2 . 6 8 6}$ & 0.9949 & 2.931 & $\mathbf{0 . 9 9 6 9}$ & $\mathbf{2 . 6 6 8}$ & 0.9953 & 2.931 \\
3S, 4S, 6S & 0.9964 & 3.395 & 0.9975 & 3.196 & 0.9964 & 3.447 & 0.9978 & 3.285 \\
3R, 4R, 6S & 0.9966 & 2.717 & $\mathbf{0 . 9 9 8 0}$ & $\mathbf{2 . 5 5 1}$ & 0.9966 & 2.758 & $\mathbf{0 . 9 9 8 2}$ & $\mathbf{2 . 5 7 4}$ \\
\hline
\end{tabular}

Table S20 DP4+ probabilities calculated for assignment of calculated data

\begin{tabular}{ccccc}
\hline & $\mathbf{5}$ & $\mathbf{6}$ & $\mathbf{7}$ & $\mathbf{8}$ \\
\hline 3R, 4S, 6S & $9.72 \%$ & $0.00 \%$ & $24.64 \%$ & $0.00 \%$ \\
3S, 4R, 6S & $\mathbf{8 8 . 3 5 \%}$ & $0.00 \%$ & $\mathbf{6 4 . 8 1 \%}$ & $0.00 \%$ \\
3S, 4S, 6S & $1.38 \%$ & $20.19 \%$ & $5.78 \%$ & $37.54 \%$ \\
3R, 4R, 6S & $0.55 \%$ & $\mathbf{7 9 . 8 1 \%}$ & $4.77 \%$ & $\mathbf{6 2 . 4 6 \%}$ \\
\hline
\end{tabular}


Table S21 ${ }^{13} \mathrm{C}$ NMR MAE ${ }_{\Delta} \delta$ values of the accounted comparison alignments of fixed sequence calc_a calc_b calc_c calc_d and all possible combinations of exp_5 exp_6 exp_7 exp_8 (the bold data set implies lowest $\mathrm{MAE}_{\Delta \Delta \delta}$ and the fittest sequence)

\begin{tabular}{|c|c|c|c|c|}
\hline \multicolumn{4}{|c|}{ Fixed sequences } & \multirow{2}{*}{$\frac{\mathrm{MAE}_{\Delta \Delta \delta}}{0.5807}$} \\
\hline exp_5 & exp_6 & exp_7 & exp_8 & \\
\hline exp_7 & exp_6 & exp_5 & exp_8 & 0.5981 \\
\hline exp_5 & exp_8 & exp_7 & exp_6 & 0.6364 \\
\hline exp_7 & exp_8 & exp_5 & exp_6 & 0.6546 \\
\hline exp_8 & exp_5 & exp_7 & exp_6 & 1.0919 \\
\hline exp_7 & exp_5 & exp_6 & exp_8 & 1.1029 \\
\hline exp_6 & exp_5 & exp_7 & exp_8 & 1.1091 \\
\hline exp_7 & exp_5 & exp_8 & exp_6 & 1.1132 \\
\hline exp_8 & exp_6 & exp_7 & exp_5 & 1.1275 \\
\hline exp_5 & exp_7 & exp_6 & exp_8 & 1.1370 \\
\hline exp_8 & exp_6 & exp_5 & exp_7 & 1.1428 \\
\hline exp_7 & exp_6 & exp_8 & exp_5 & 1.1448 \\
\hline exp_8 & exp_7 & exp_5 & exp_6 & 1.1475 \\
\hline exp_5 & exp_7 & exp_8 & exp_6 & 1.1492 \\
\hline exp_6 & exp_7 & exp_5 & exp_8 & 1.1545 \\
\hline exp_5 & exp_6 & exp_8 & exp_7 & 1.1619 \\
\hline exp_6 & exp_8 & exp_7 & exp_5 & 1.1726 \\
\hline exp_6 & exp_8 & exp_5 & exp_7 & 1.1880 \\
\hline exp_7 & exp_8 & exp_6 & exp_5 & 1.1923 \\
\hline exp_5 & exp_8 & exp_6 & exp_7 & 1.2083 \\
\hline exp_8 & exp_5 & exp_6 & exp_7 & 1.3499 \\
\hline exp_6 & exp_5 & exp_8 & exp_7 & 1.3553 \\
\hline exp_6 & exp_7 & exp_8 & exp_5 & 1.3857 \\
\hline exp_8 & exp_7 & exp_6 & exp_5 & 1.3888 \\
\hline
\end{tabular}


Fig. S7 Experimental and calculated CD spectra of compounds 5 and 7 (A), 6 and 8 (B)

A

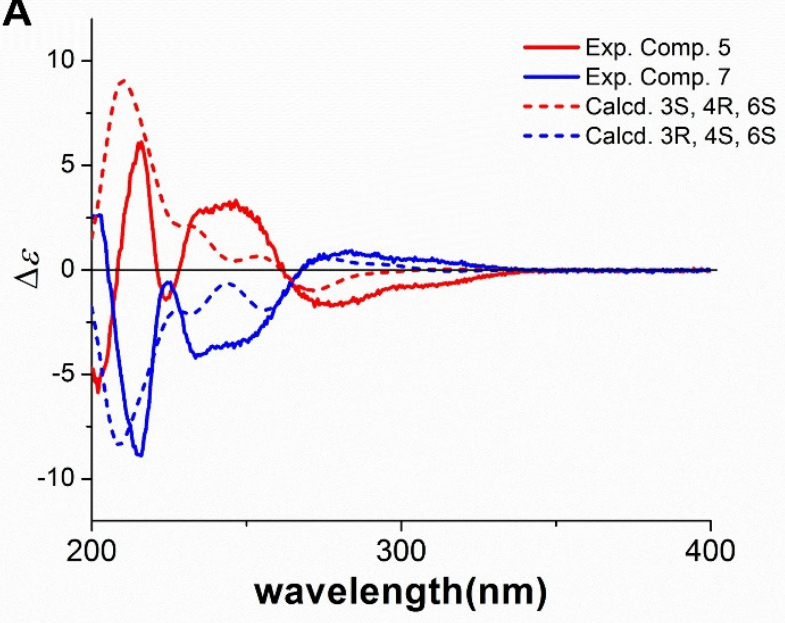

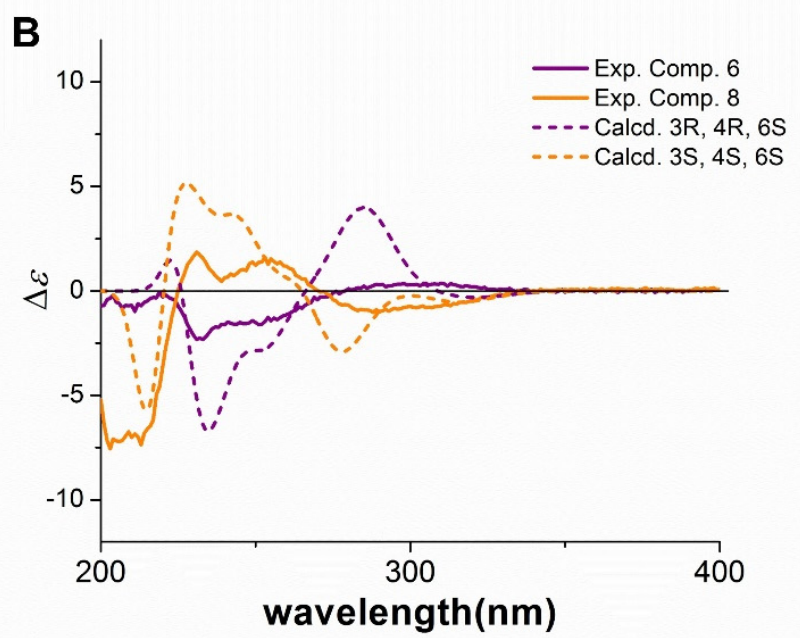

Fig. S8 CD spectrum of F-14329 (2) (left), and reported data (right) ${ }^{9}$
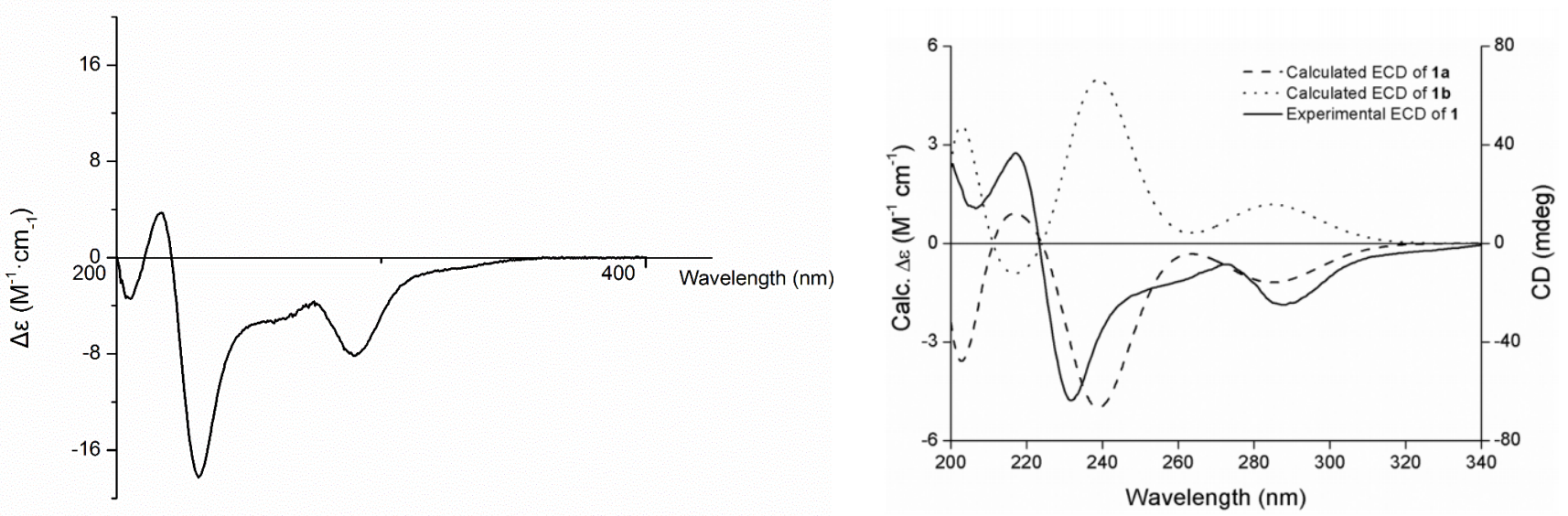

Fig. S9 CD spectrum of compounds 3 and 4

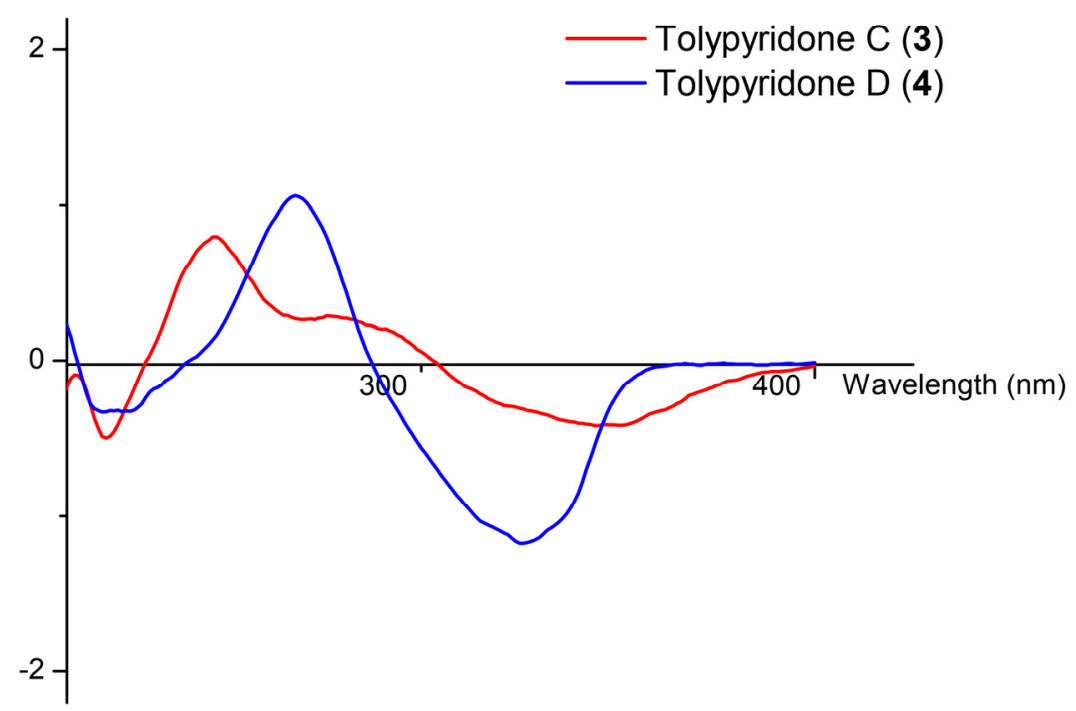




\section{NMR and MS spectra}

Fig. S10 ${ }^{1} \mathrm{H}$ NMR spectrum of compound 3
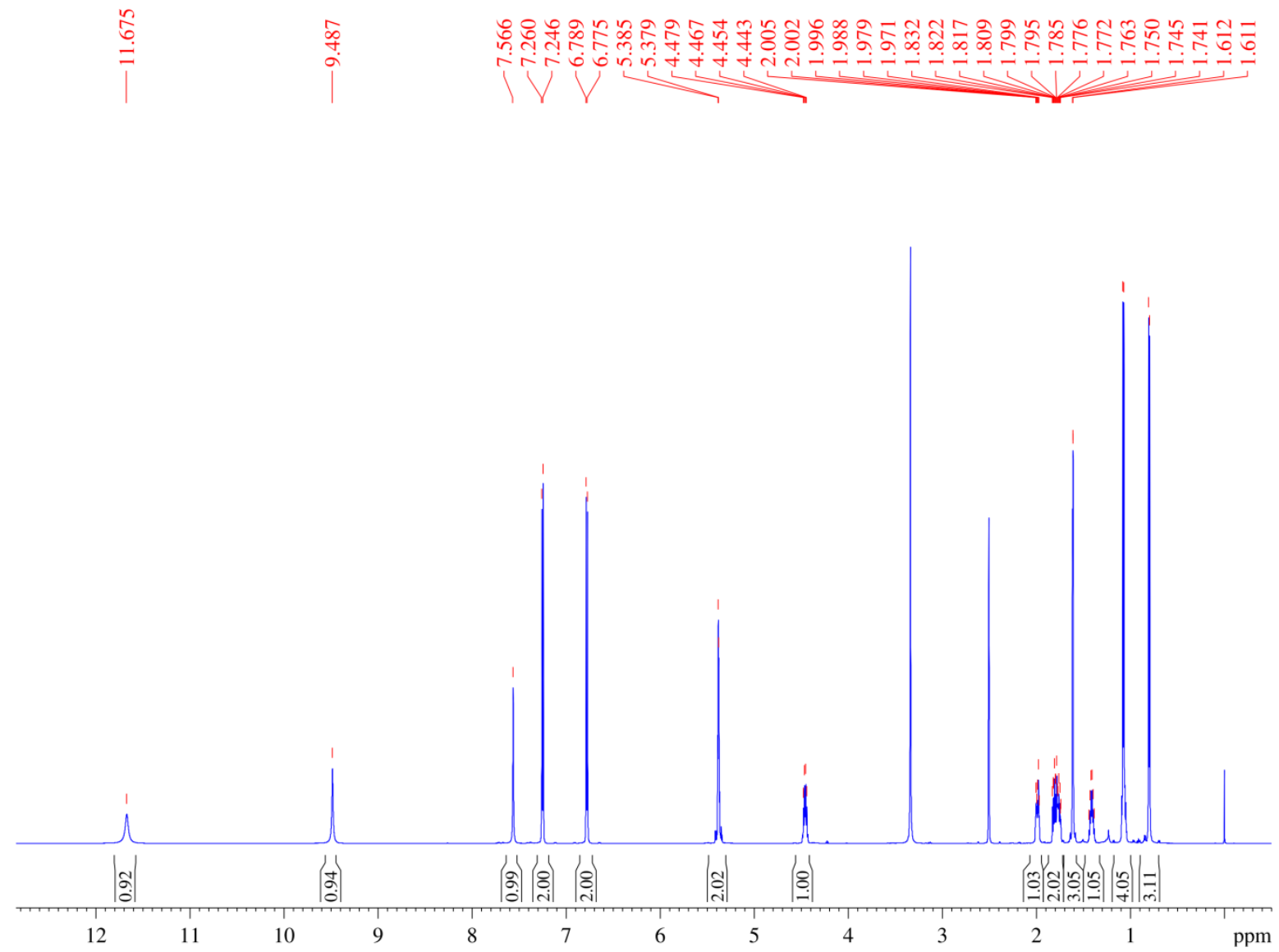

Fig. S11 ${ }^{13} \mathrm{C}$ NMR spectrum of compound 3

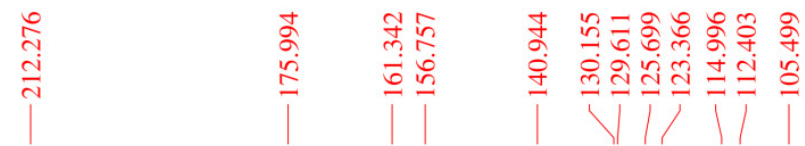

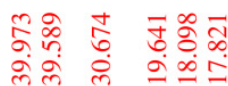

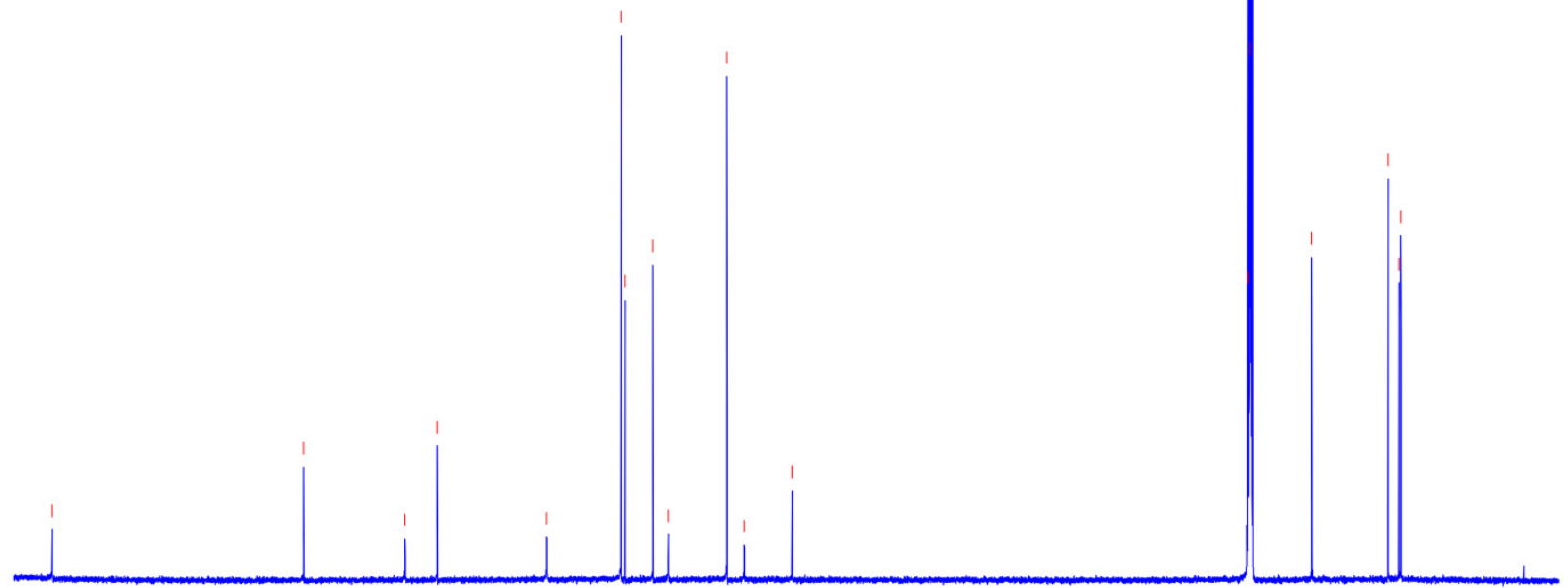

$\begin{array}{llllllllllllllllllllll}210 & 200 & 190 & 180 & 170 & 160 & 150 & 140 & 130 & 120 & 110 & 100 & 90 & 80 & 70 & 60 & 50 & 40 & 30 & 20 & 10 & \text { ppm }\end{array}$ 
Fig. S12 HSQC spectrum of compound 3

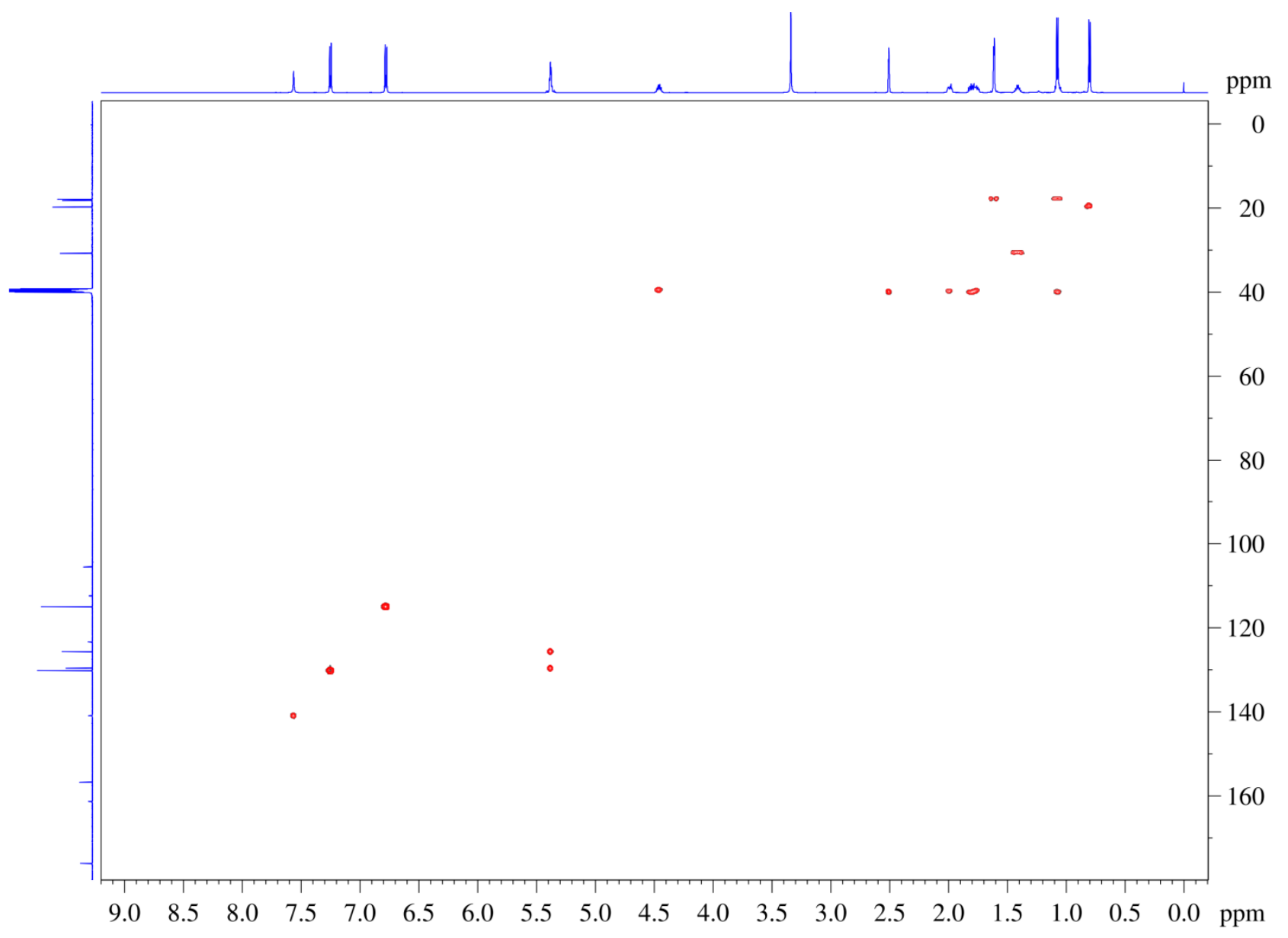

Fig. S13 HMBC spectrum of compound 3

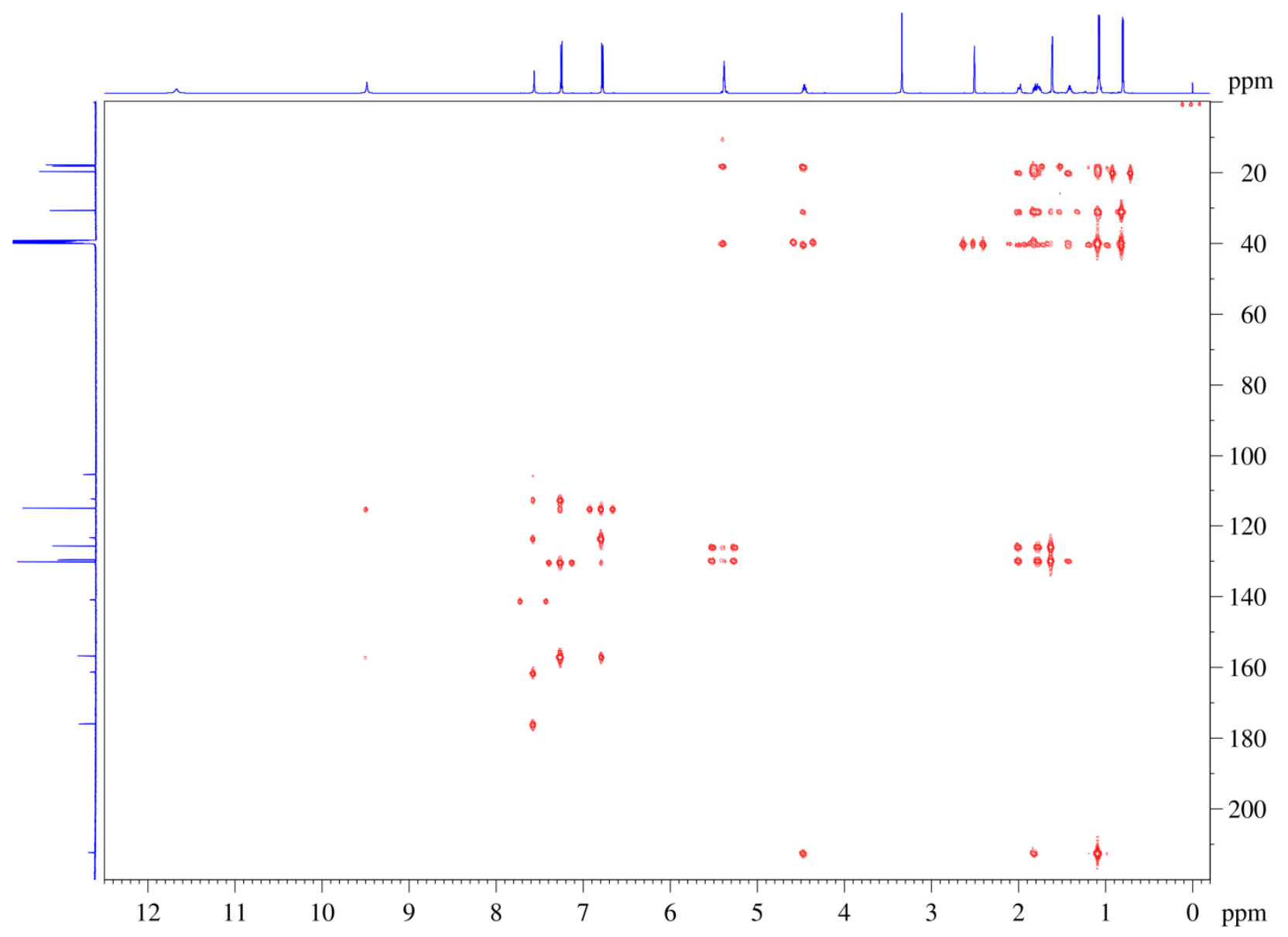


Fig. S14 ${ }^{1} \mathrm{H}-{ }^{1} \mathrm{H}$ COSY spectrum of compound 3

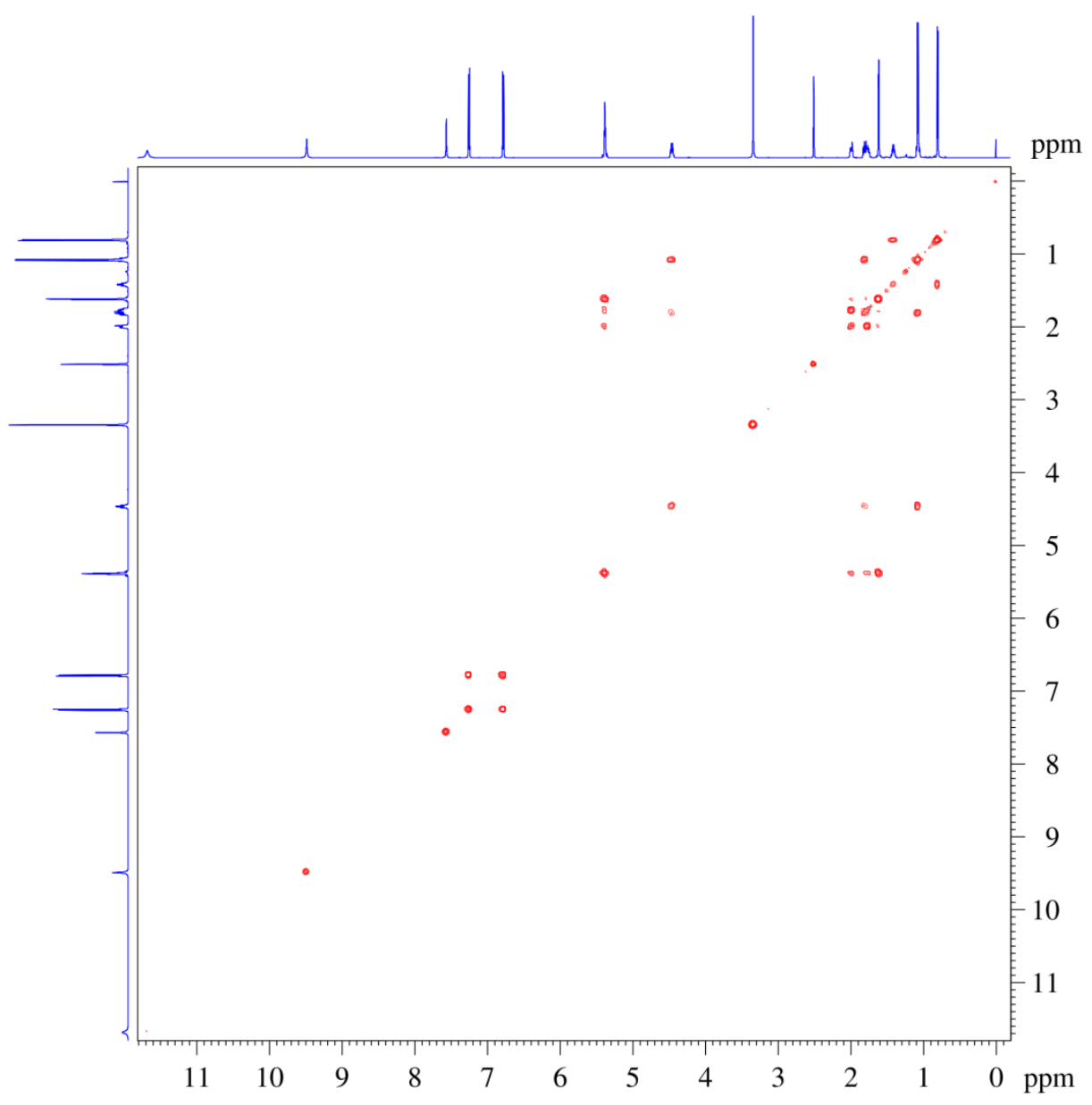

Fig. S15 ${ }^{1} \mathrm{H}$ NMR spectrum of compound 4
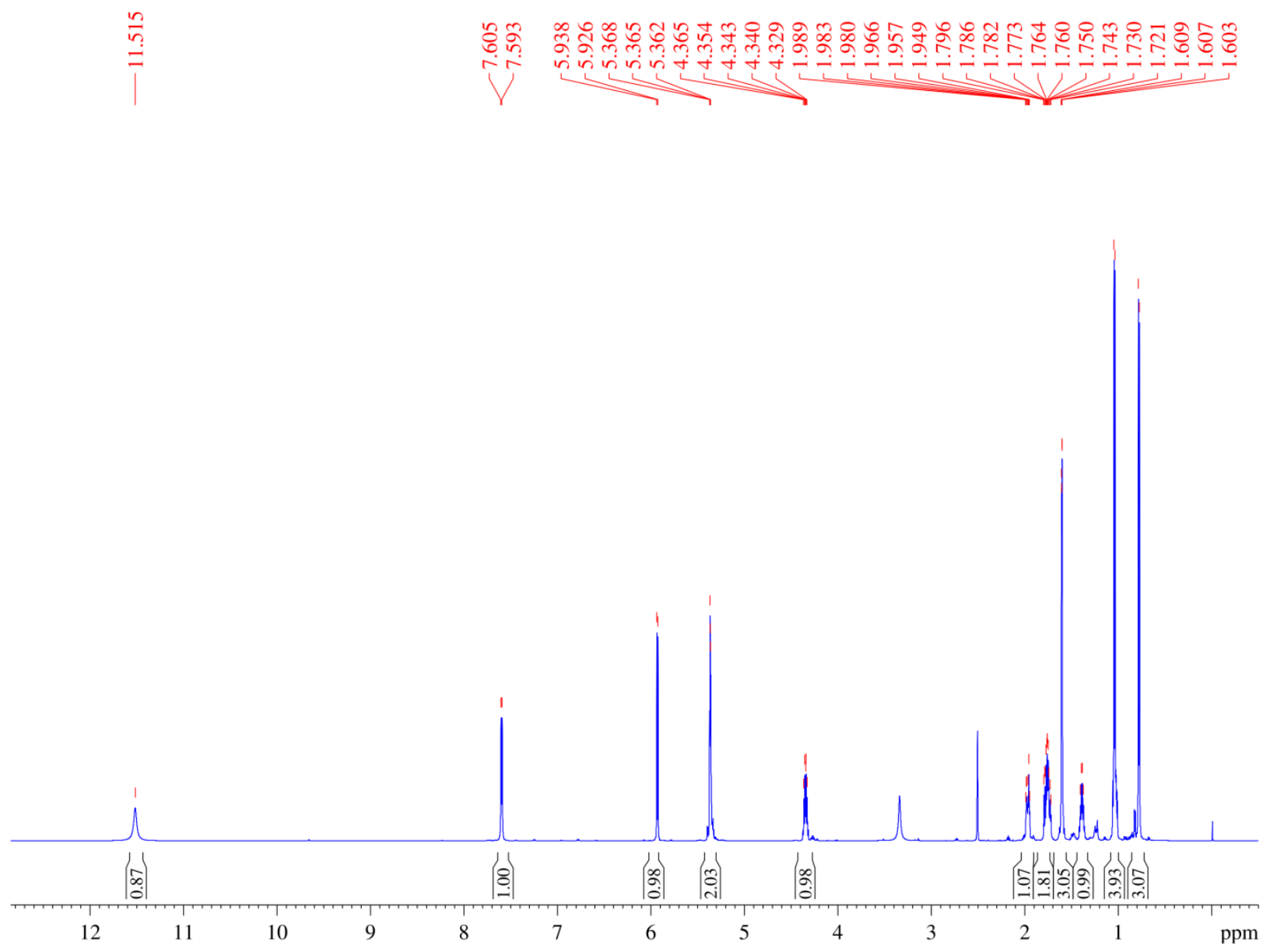
Fig. S16 ${ }^{13} \mathrm{C}$ NMR spectrum of compound 4

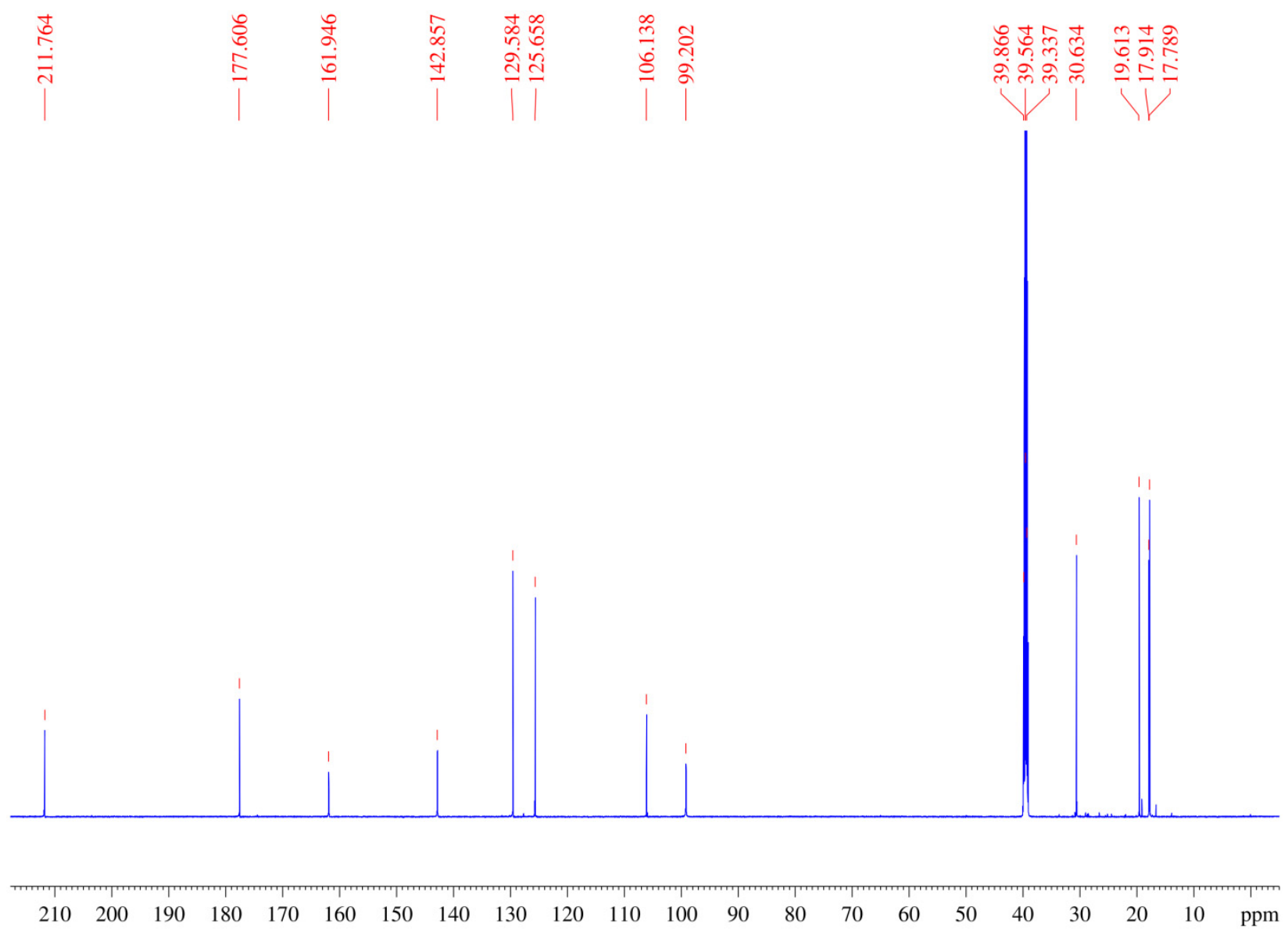

Fig. S17 HSQC spectrum of compound 4

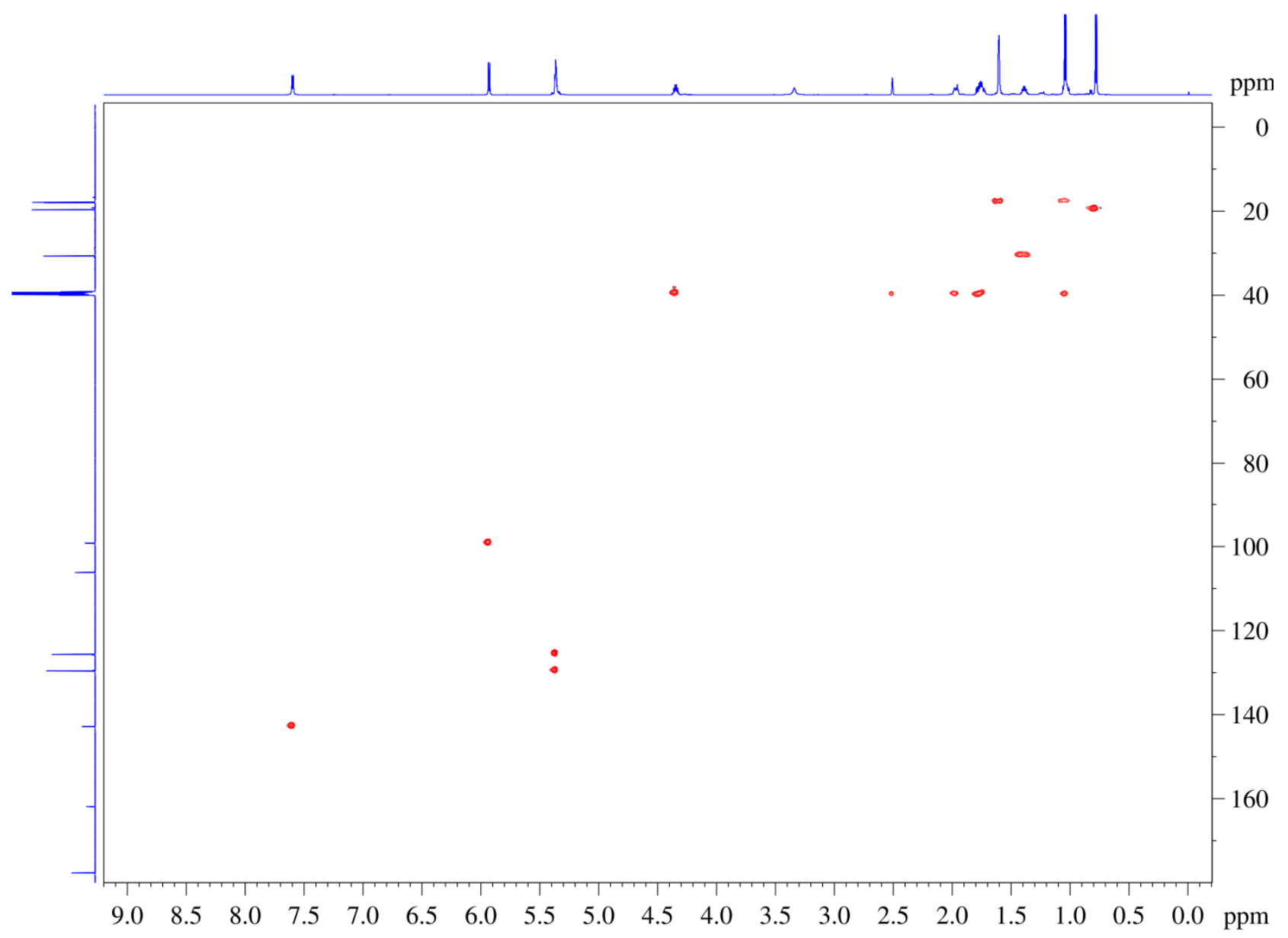


Fig. S18 HMBC spectrum of compound 4

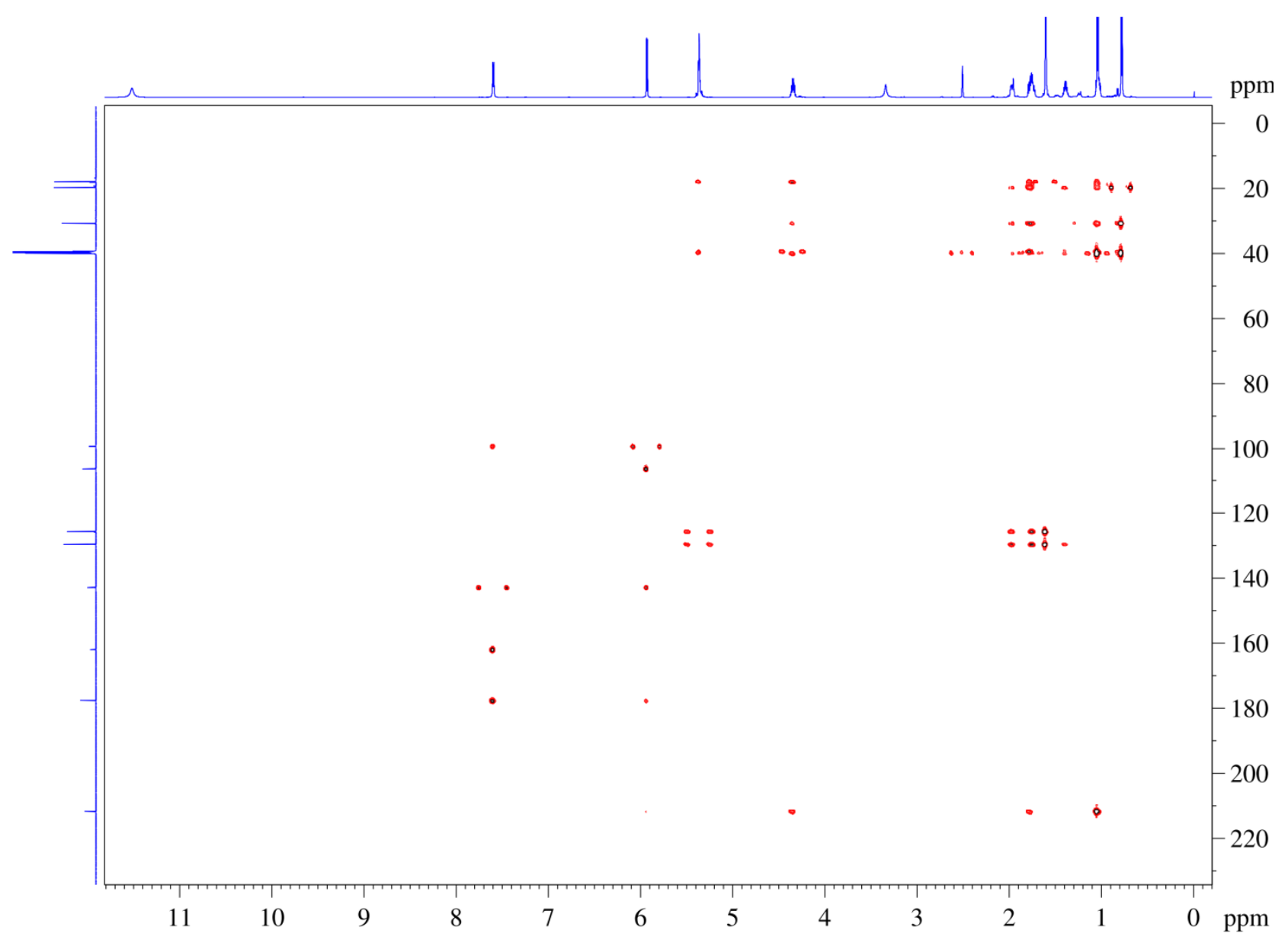

Fig. S19 ${ }^{1} \mathrm{H}-{ }^{1} \mathrm{H}$ COSY spectrum of compound 4

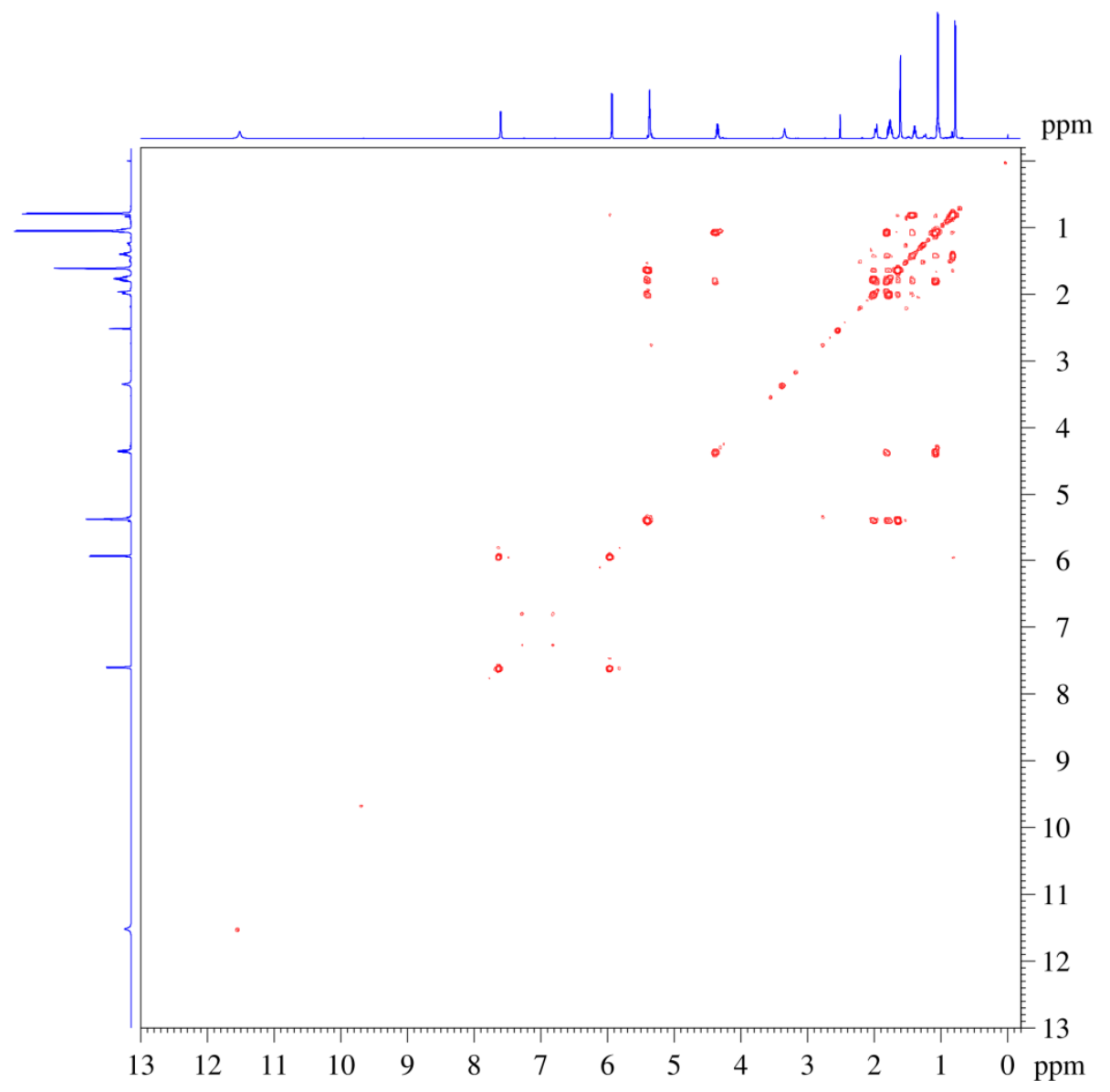


Fig. S20 ${ }^{1} \mathrm{H}$ NMR spectrum of compound 5

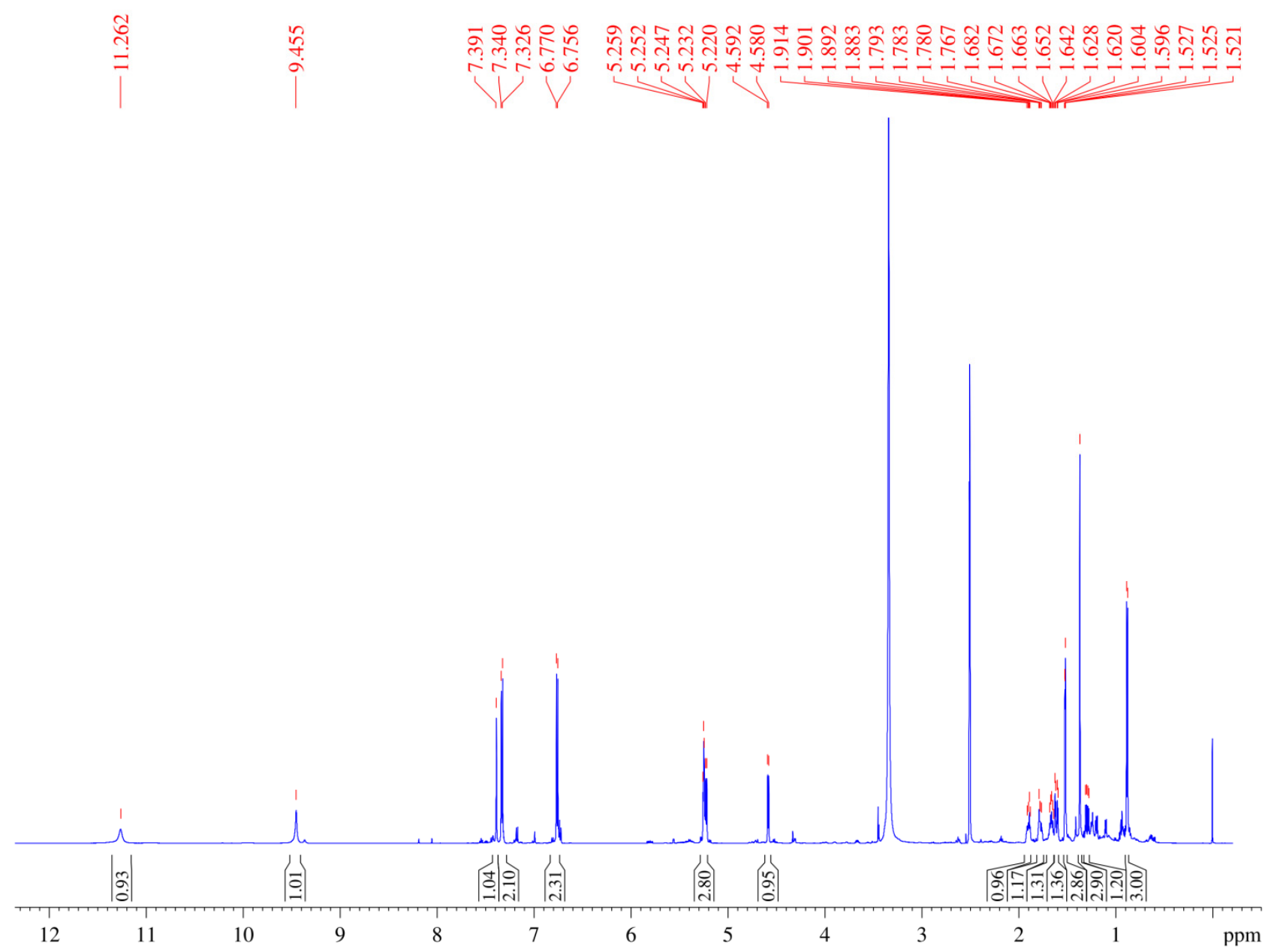

Fig. S21 13C NMR spectrum of compound 5
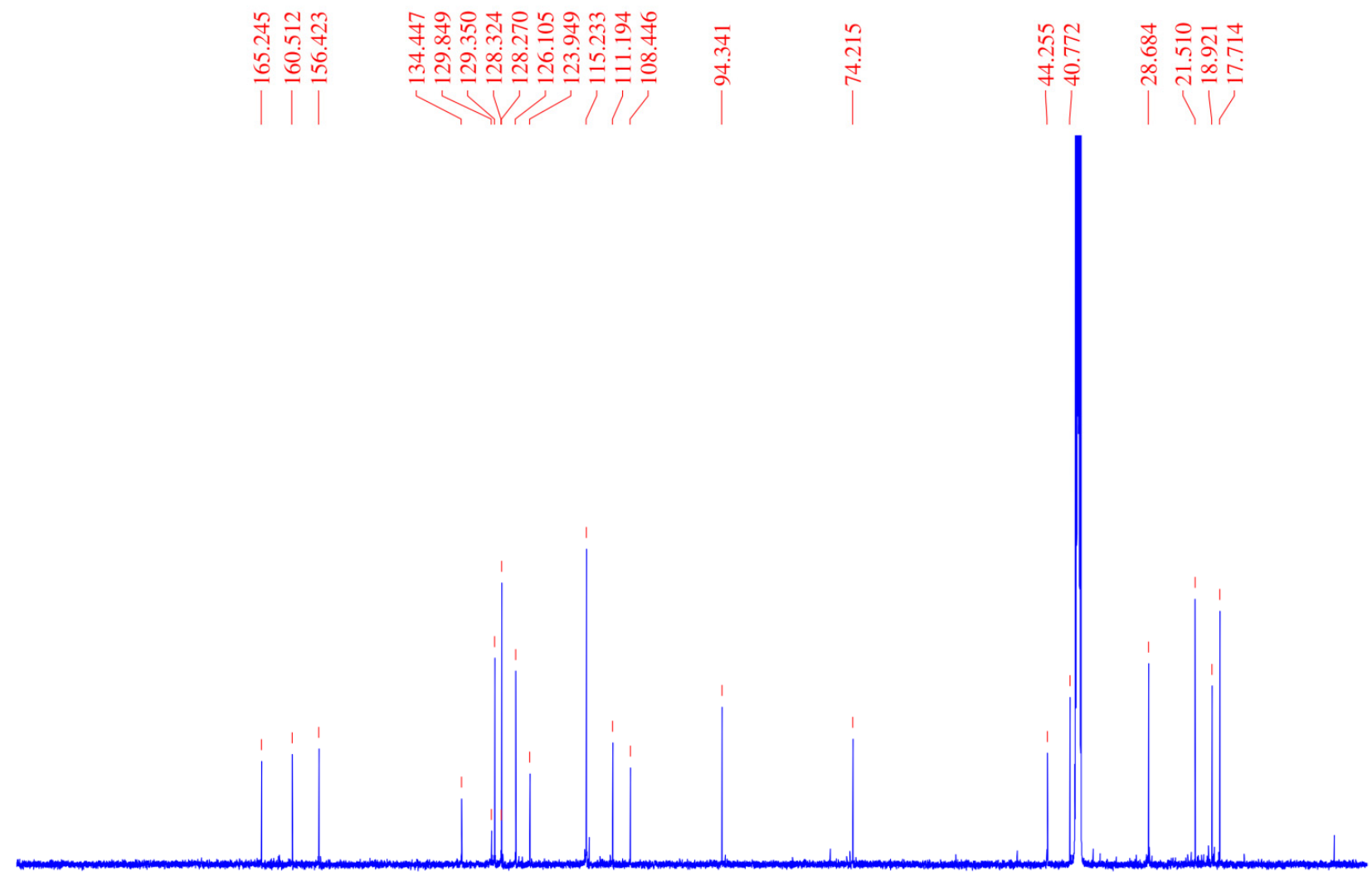

$\begin{array}{lllllllllllllllllllll}200 & 190 & 180 & 170 & 160 & 150 & 140 & 130 & 120 & 110 & 100 & 90 & 80 & 70 & 60 & 50 & 40 & 30 & 20 & 10 & \mathrm{ppm}\end{array}$ 
Fig. S22 HSQC spectrum of compound 5

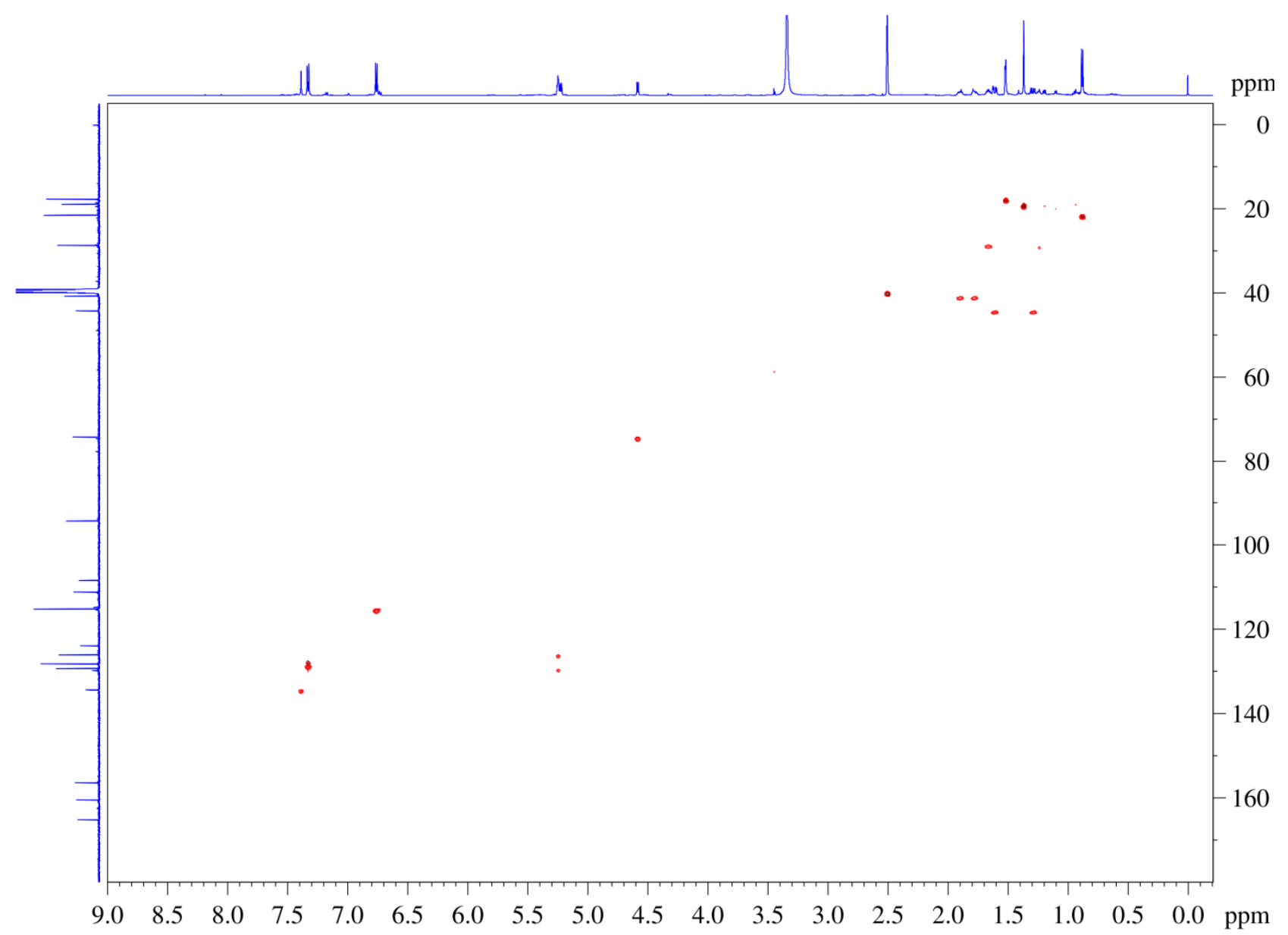

Fig. S23 HMBC spectrum of compound 5

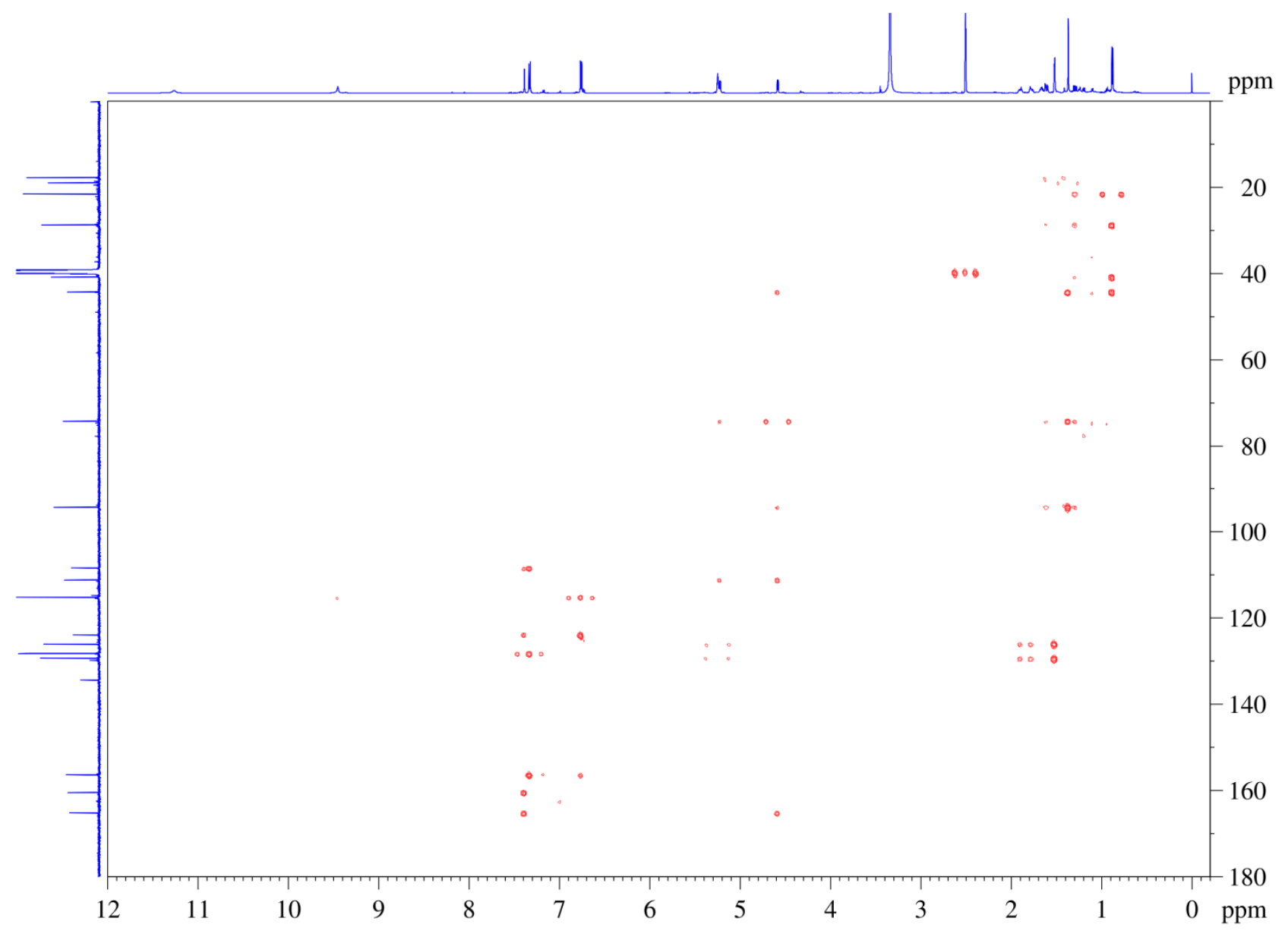


Fig. S24 ${ }^{1} \mathrm{H}-{ }^{1} \mathrm{H}$ COSY spectrum of compound 5

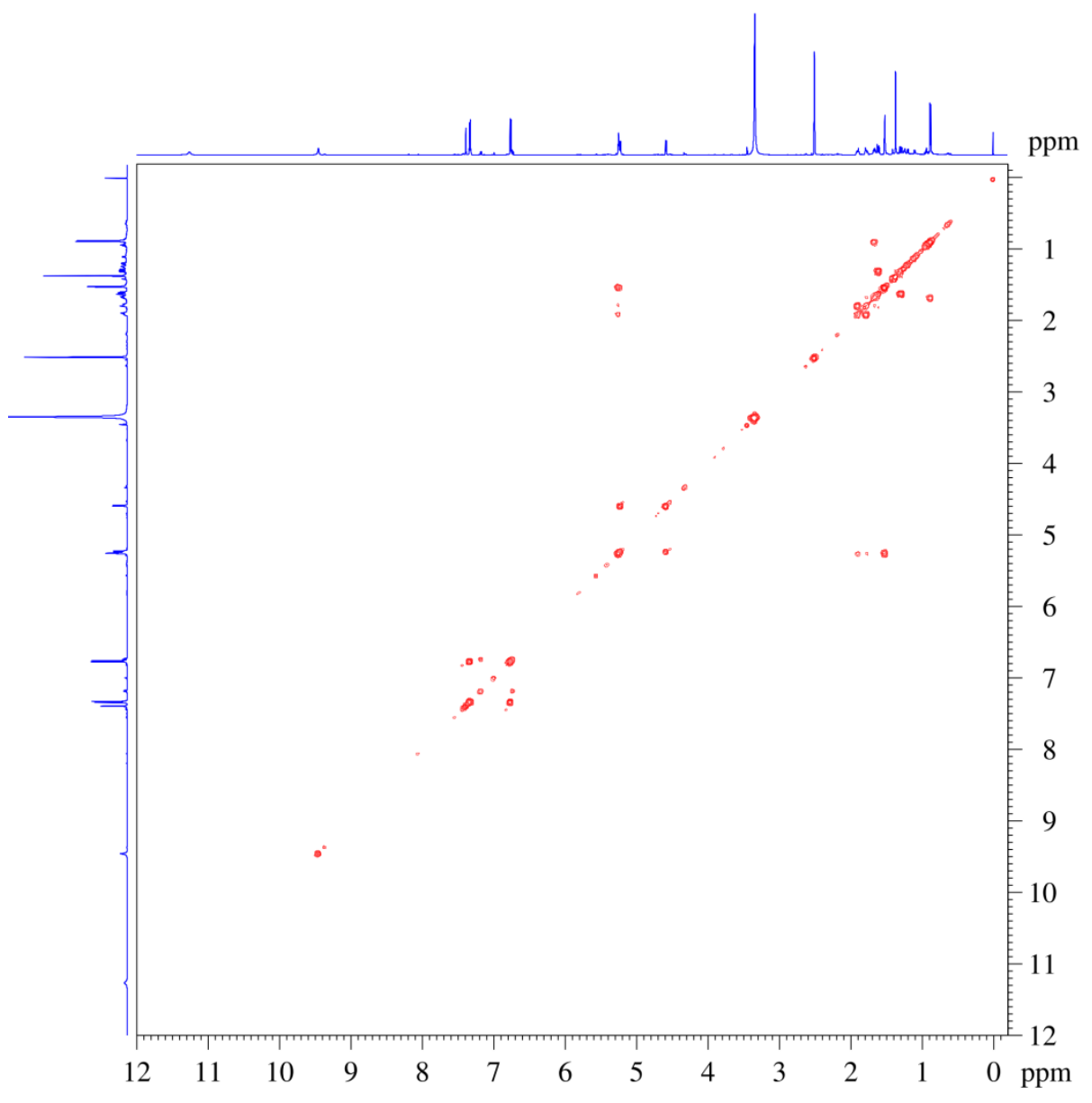

Fig. S25 ${ }^{1} \mathrm{H}$ NMR spectrum of compound 6

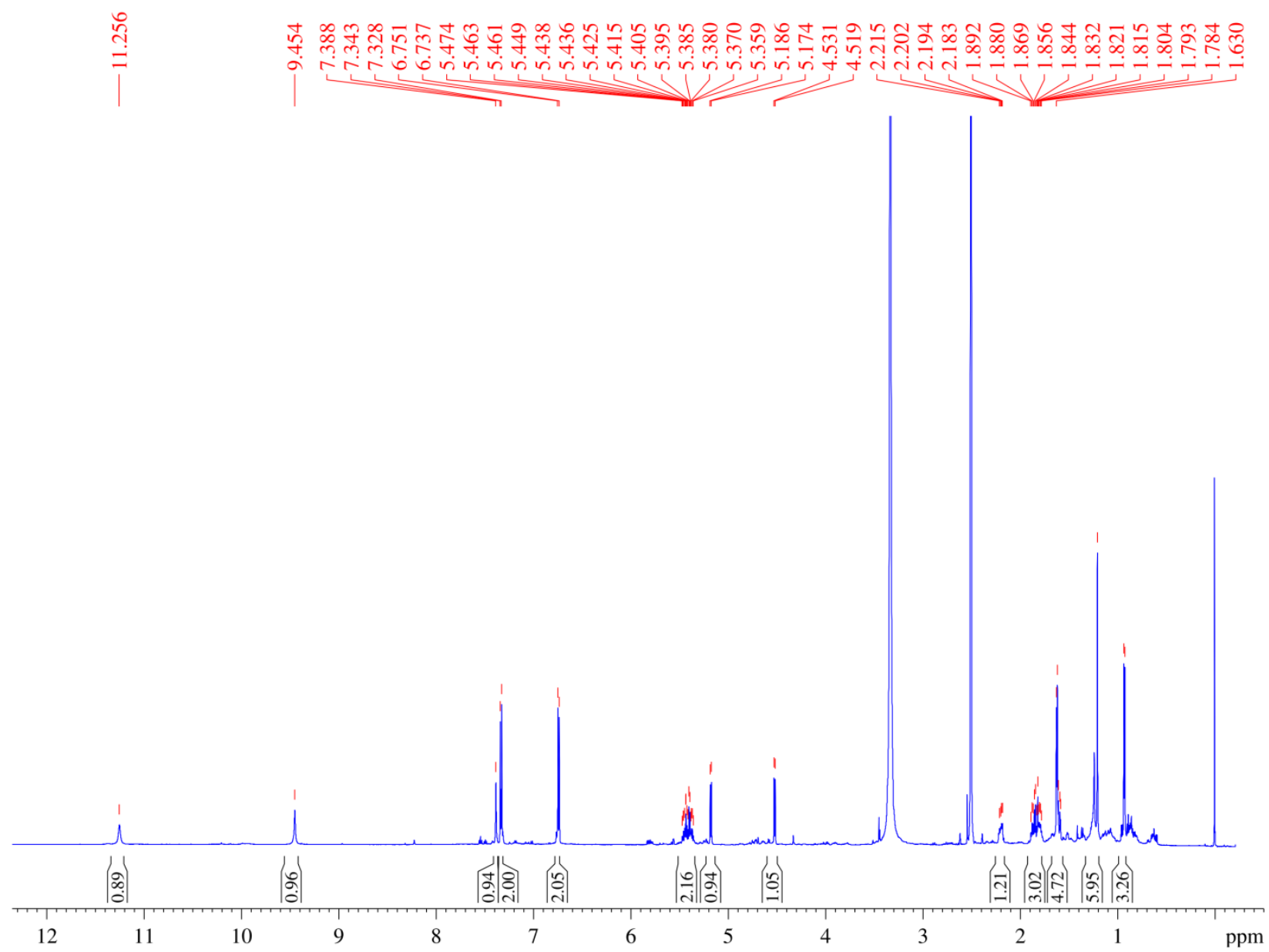


Fig. S26 ${ }^{13} \mathrm{C}$ NMR spectrum of compound 6

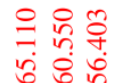

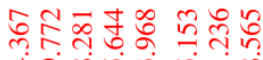

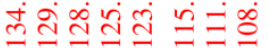

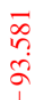

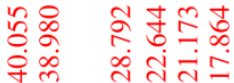
난
守
\/

$\begin{array}{llllllllllllllllllll}190 & 180 & 170 & 160 & 150 & 140 & 130 & 120 & 110 & 100 & 90 & 80 & 70 & 60 & 50 & 40 & 30 & 20 & 10 & \mathrm{ppm}\end{array}$

Fig. S27 HSQC spectrum of compound 6

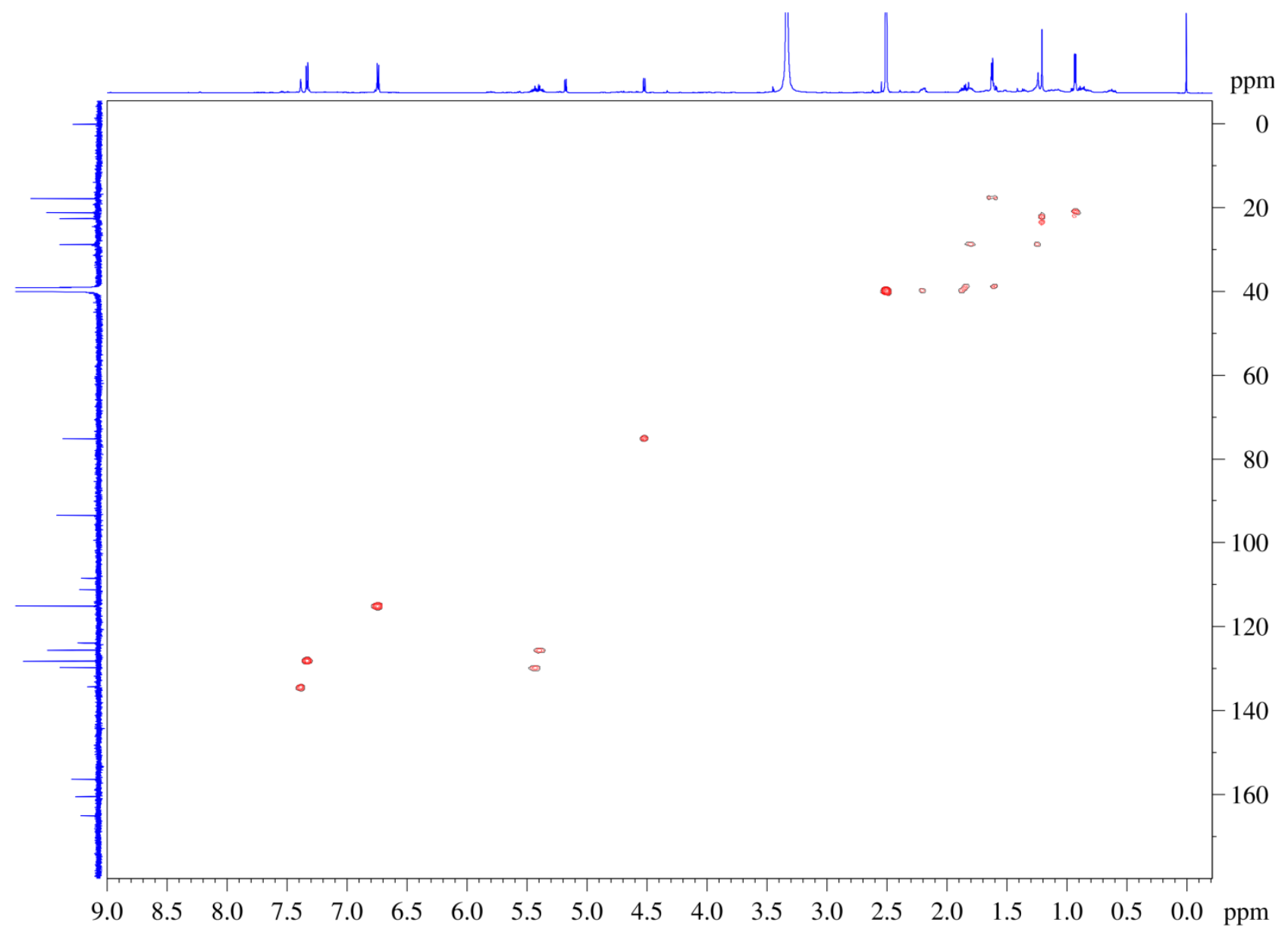


Fig. S28 HMBC spectrum of compound 6

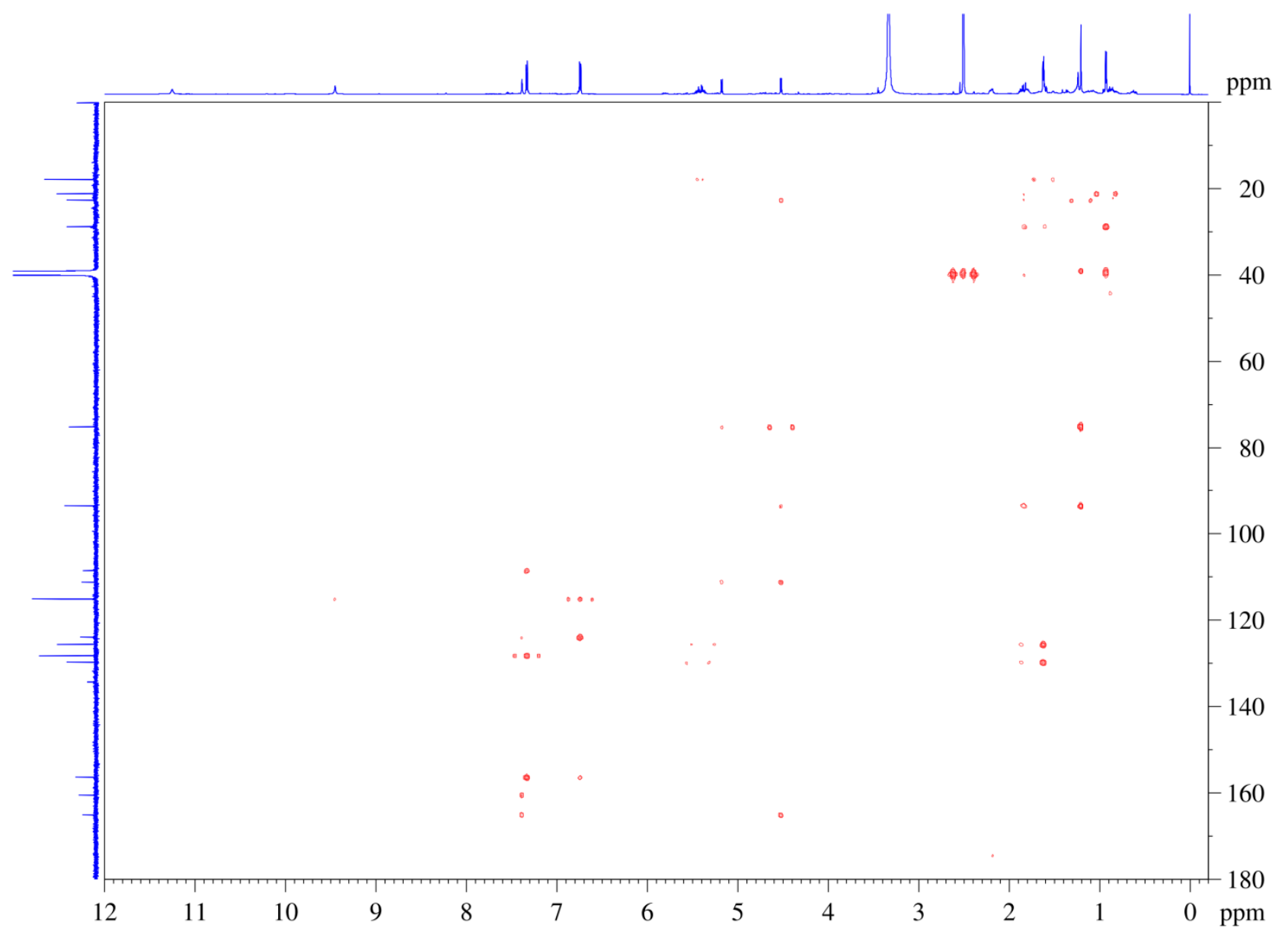

Fig. S29 ${ }^{1} \mathrm{H}-{ }^{1} \mathrm{H}$ COSY spectrum of compound 6

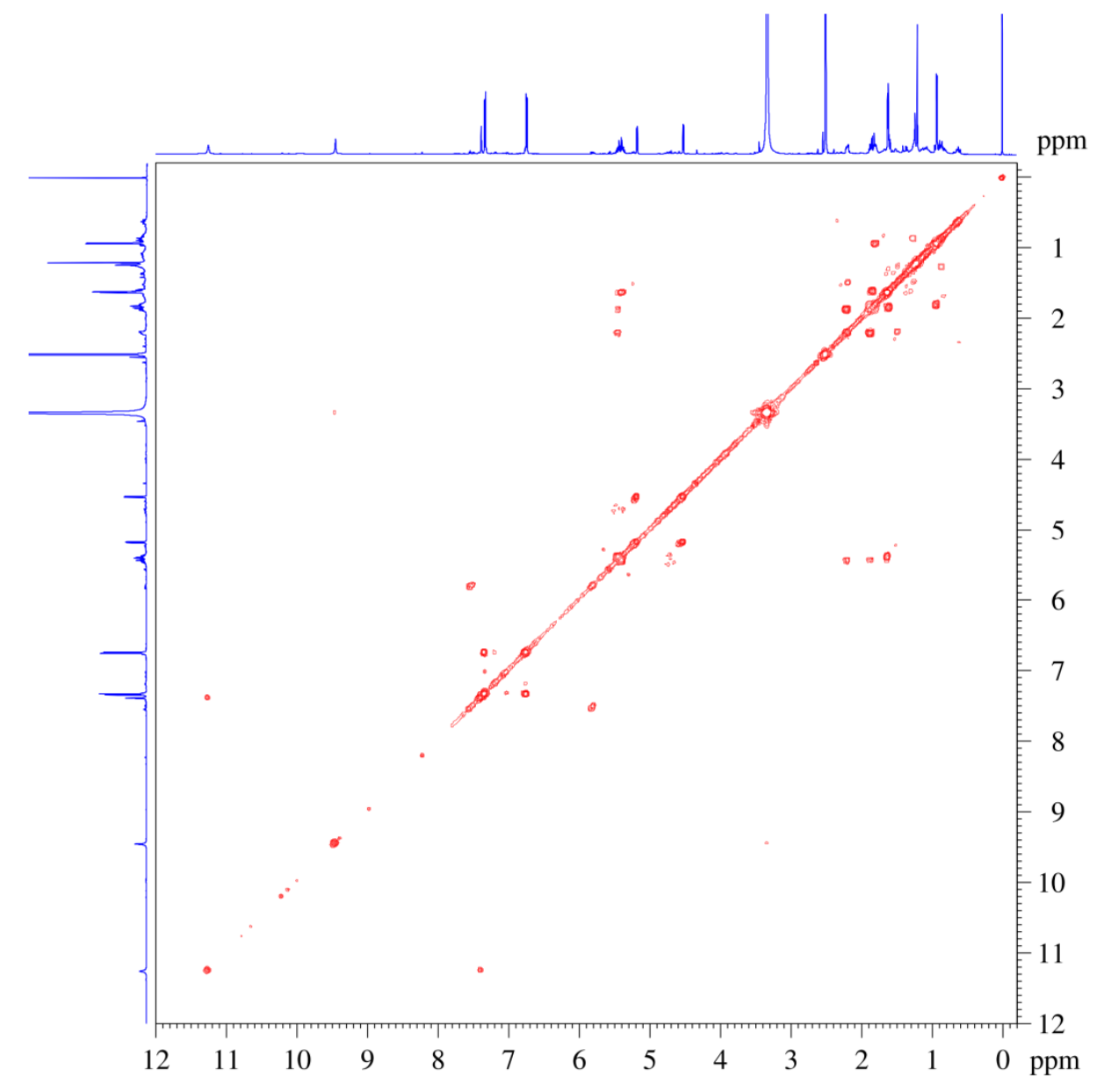


Fig. S30 ${ }^{1} \mathrm{H}$ NMR spectrum of compound 7

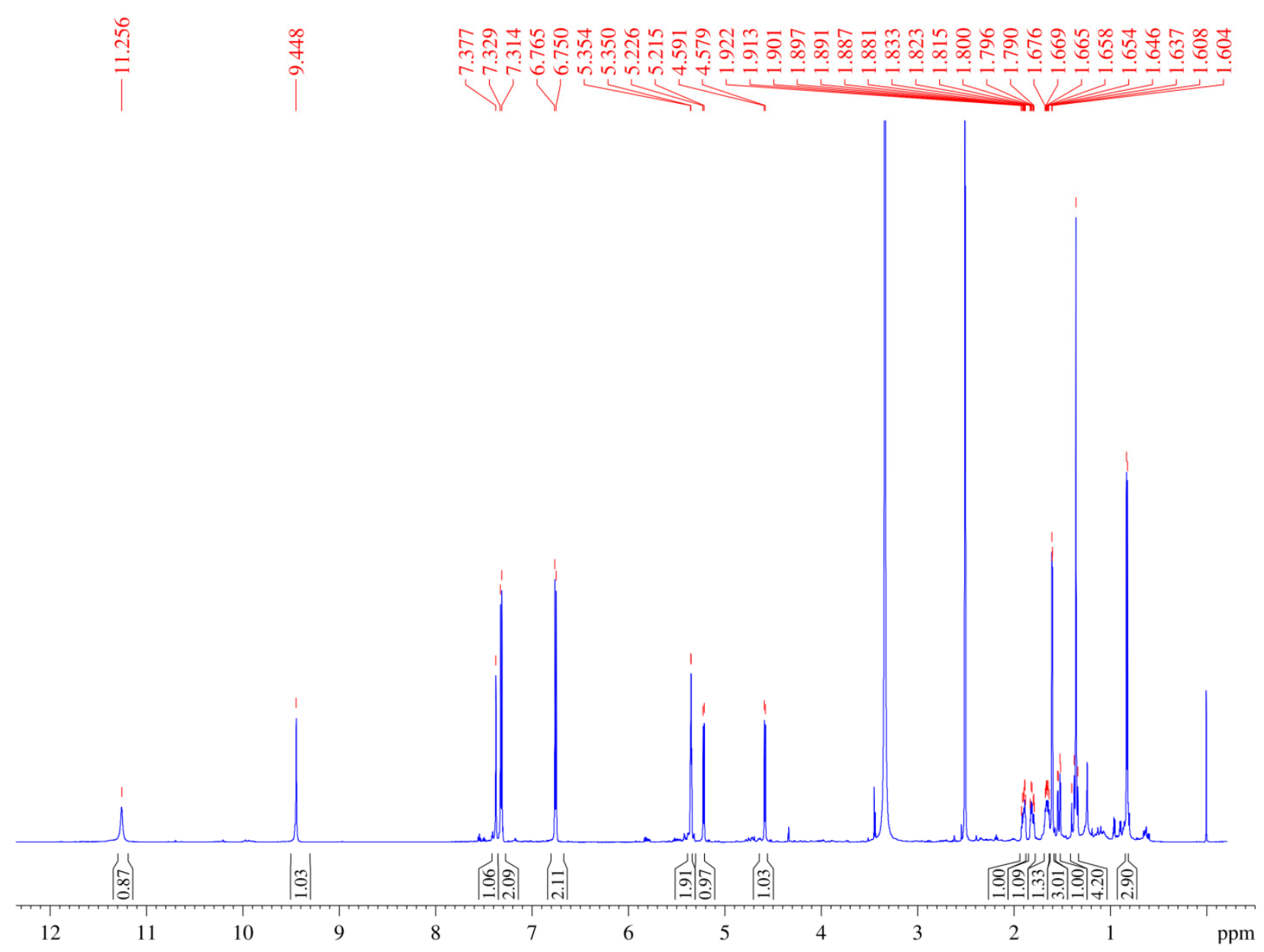

Fig. S31 ${ }^{13} \mathrm{C}$ NMR spectrum of compound 7
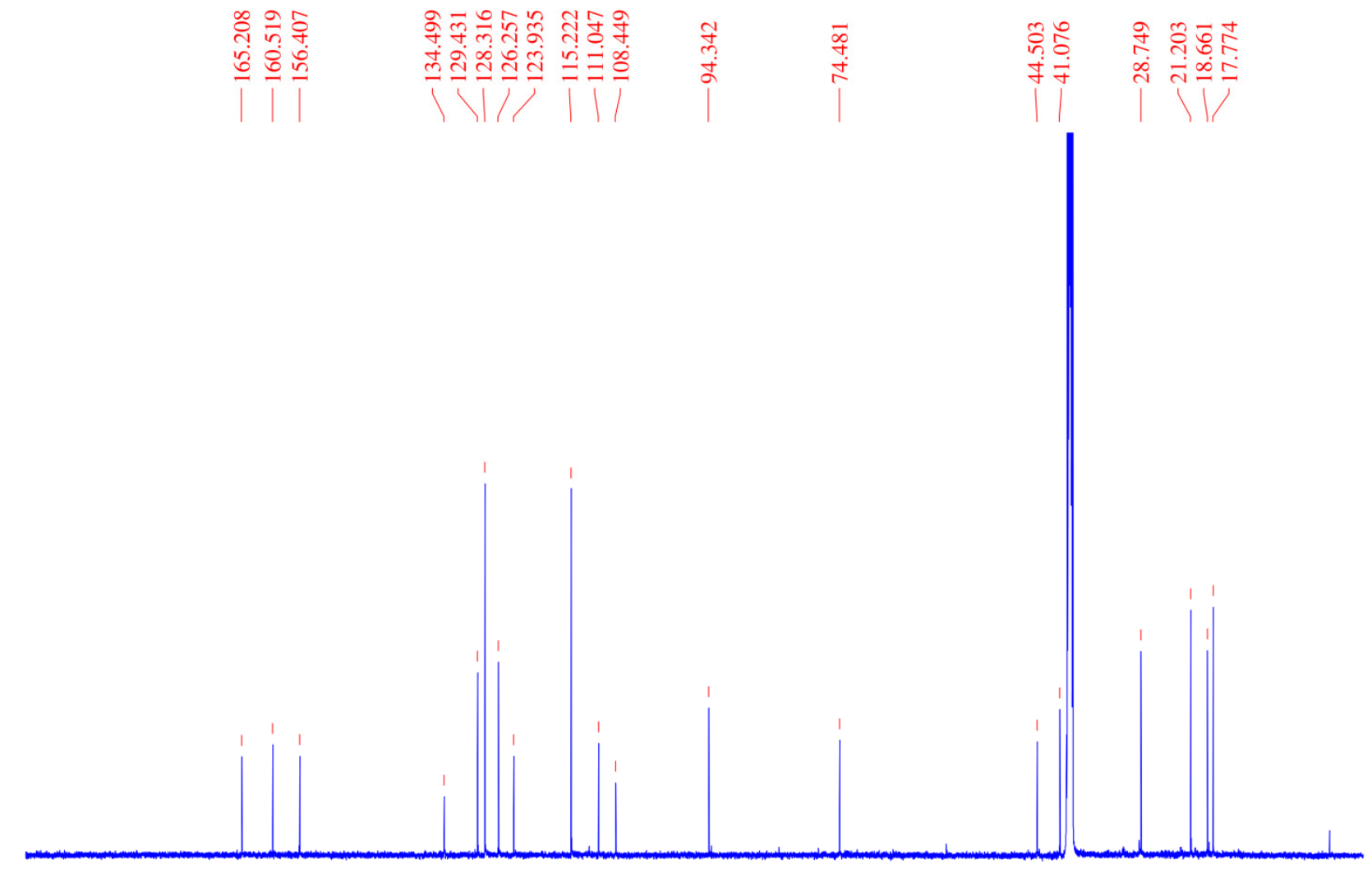

$\begin{array}{llllllllllllllllllll}190 & 180 & 170 & 160 & 150 & 140 & 130 & 120 & 110 & 100 & 90 & 80 & 70 & 60 & 50 & 40 & 30 & 20 & 10 & \mathrm{ppm}\end{array}$ 
Fig. S32 HSQC spectrum of compound 7

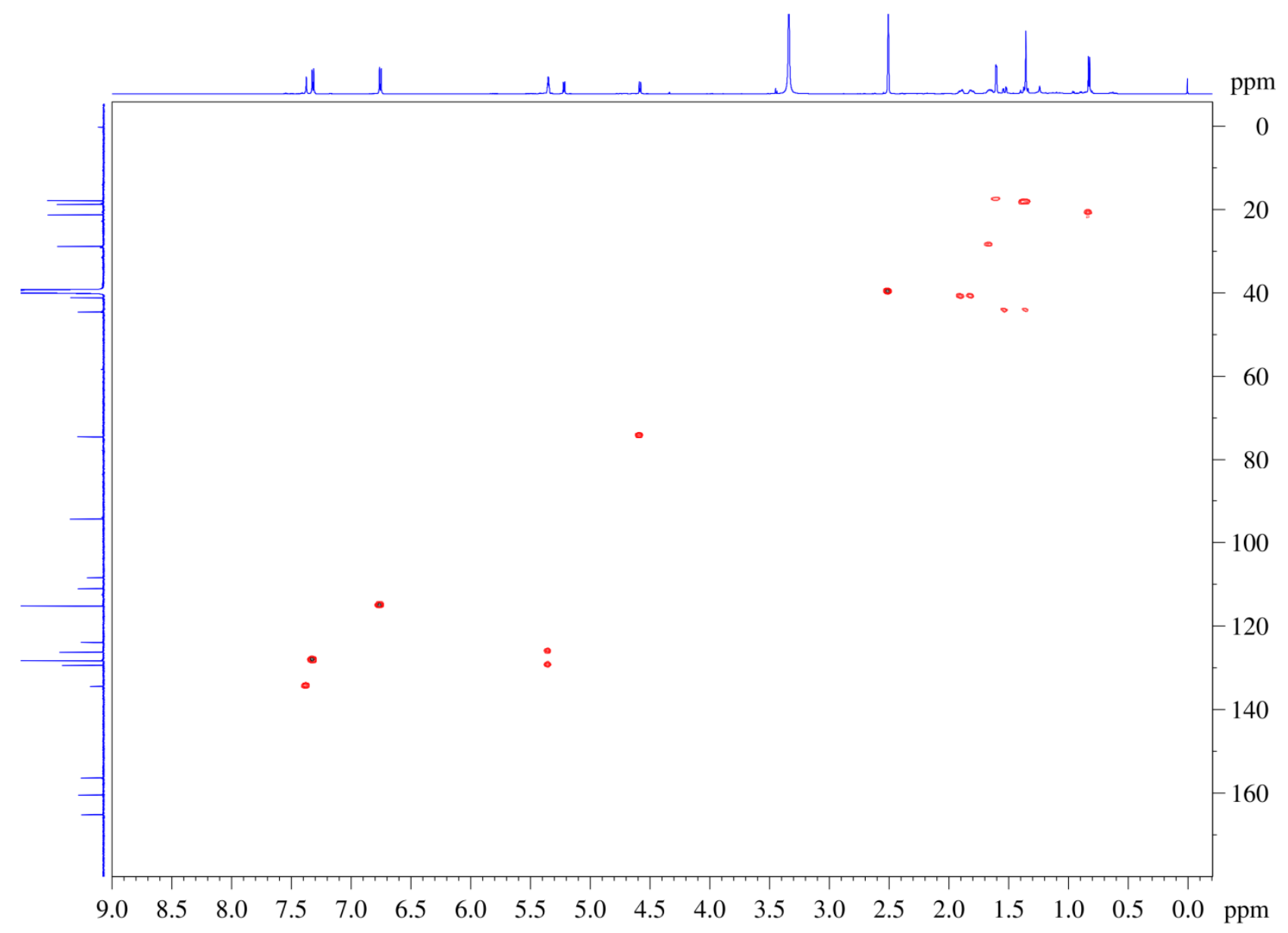

Fig. S33 HMBC spectrum of compound 7

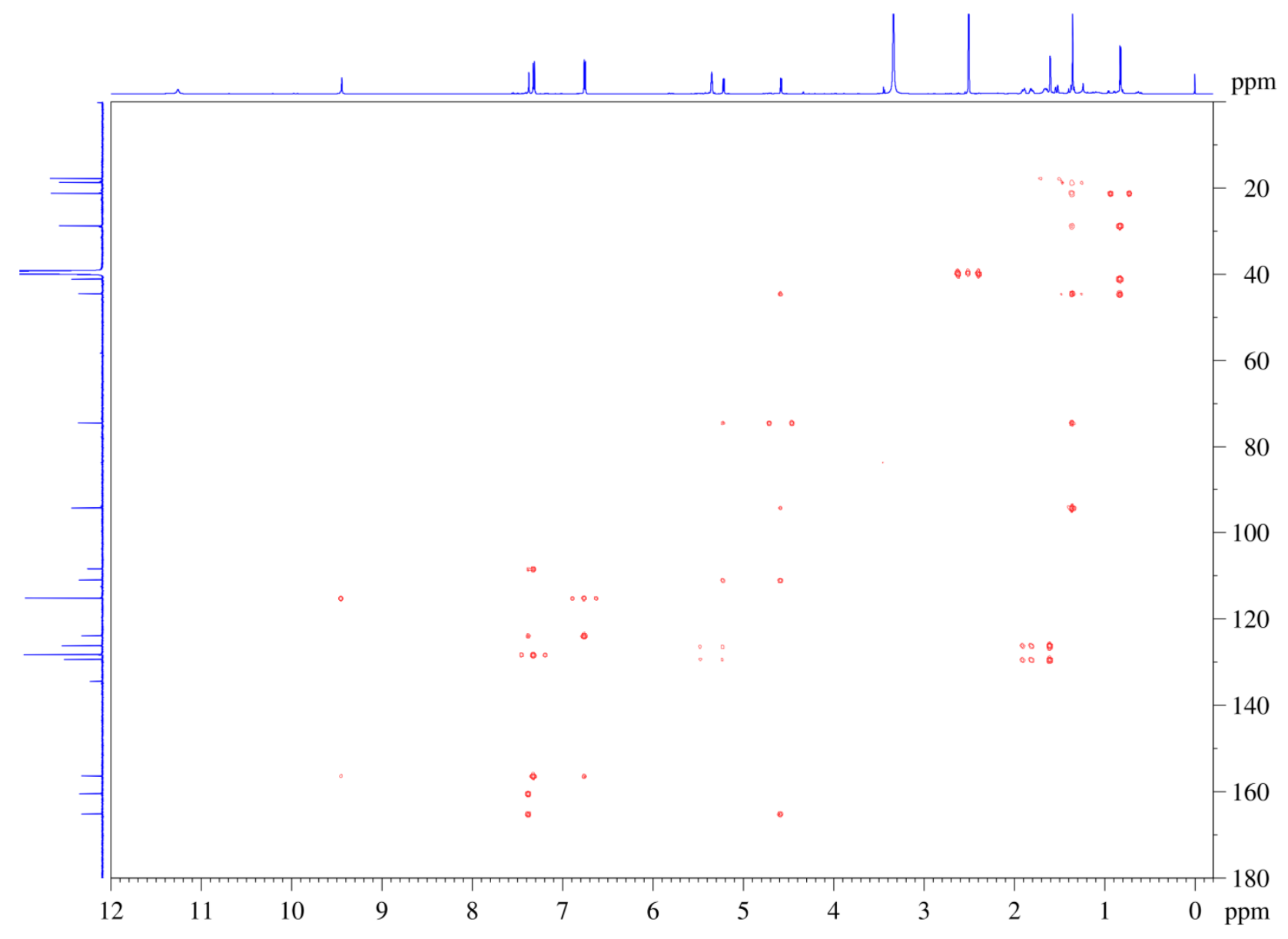


Fig. S34 ${ }^{1} \mathrm{H}-{ }^{1} \mathrm{H}$ COSY spectrum of compound 7

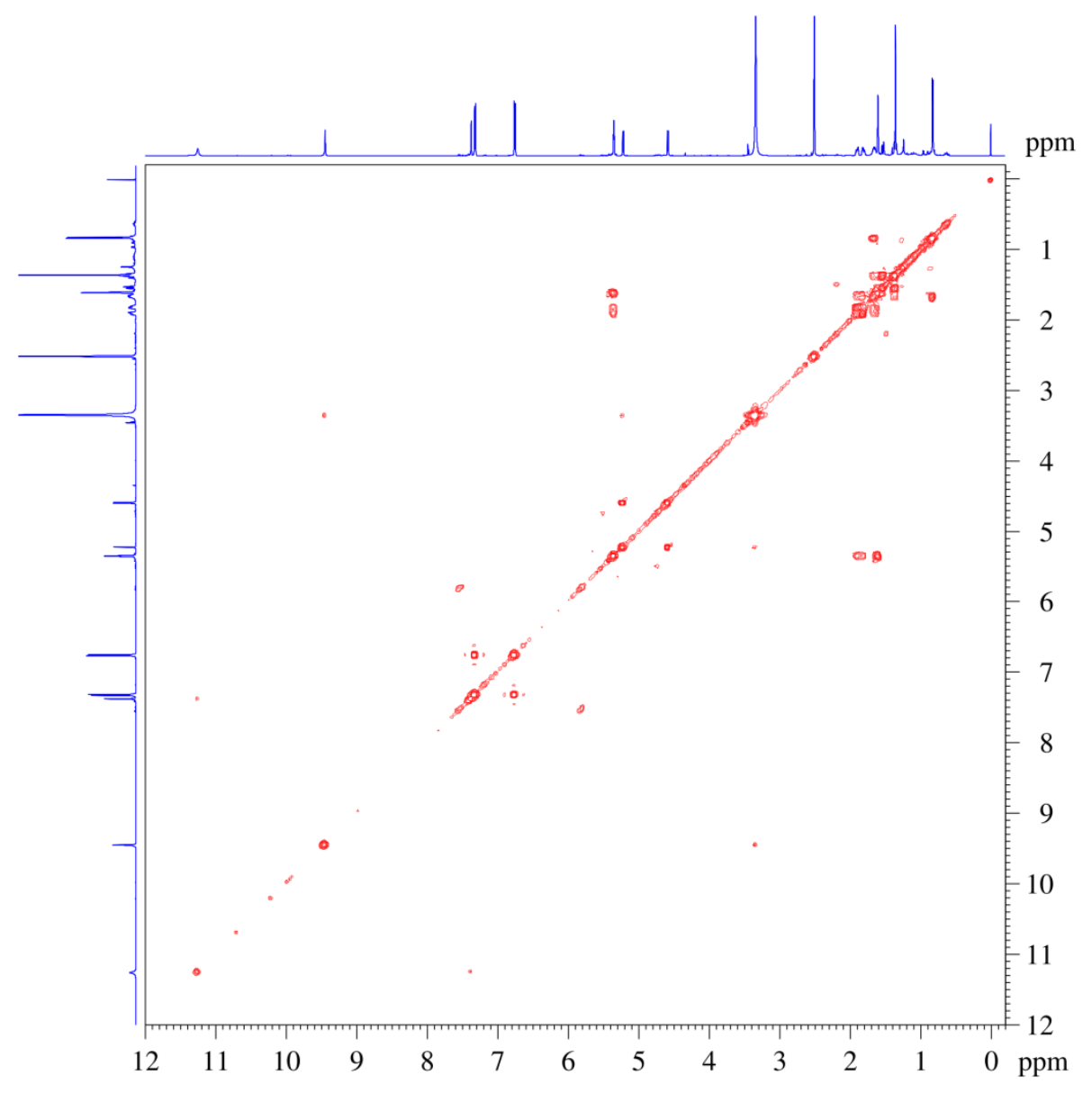

Fig. S35 ${ }^{1}$ H NMR spectrum of compound 8

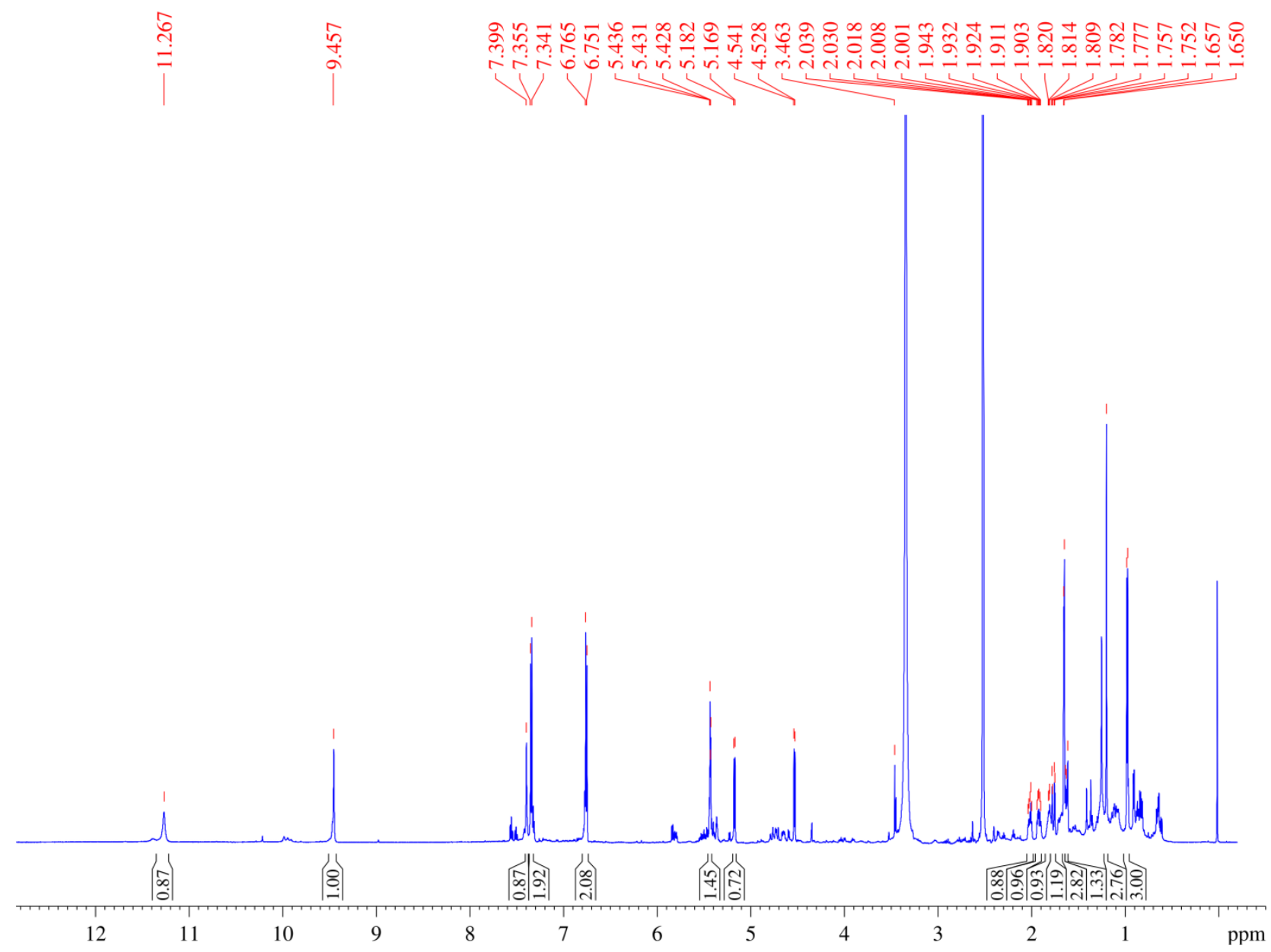


Fig. $S 36{ }^{13} \mathrm{C}$ NMR spectrum of compound 8

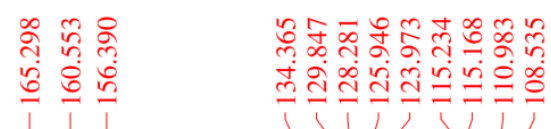

ڤ̊

$\stackrel{n}{n}$

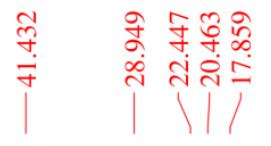

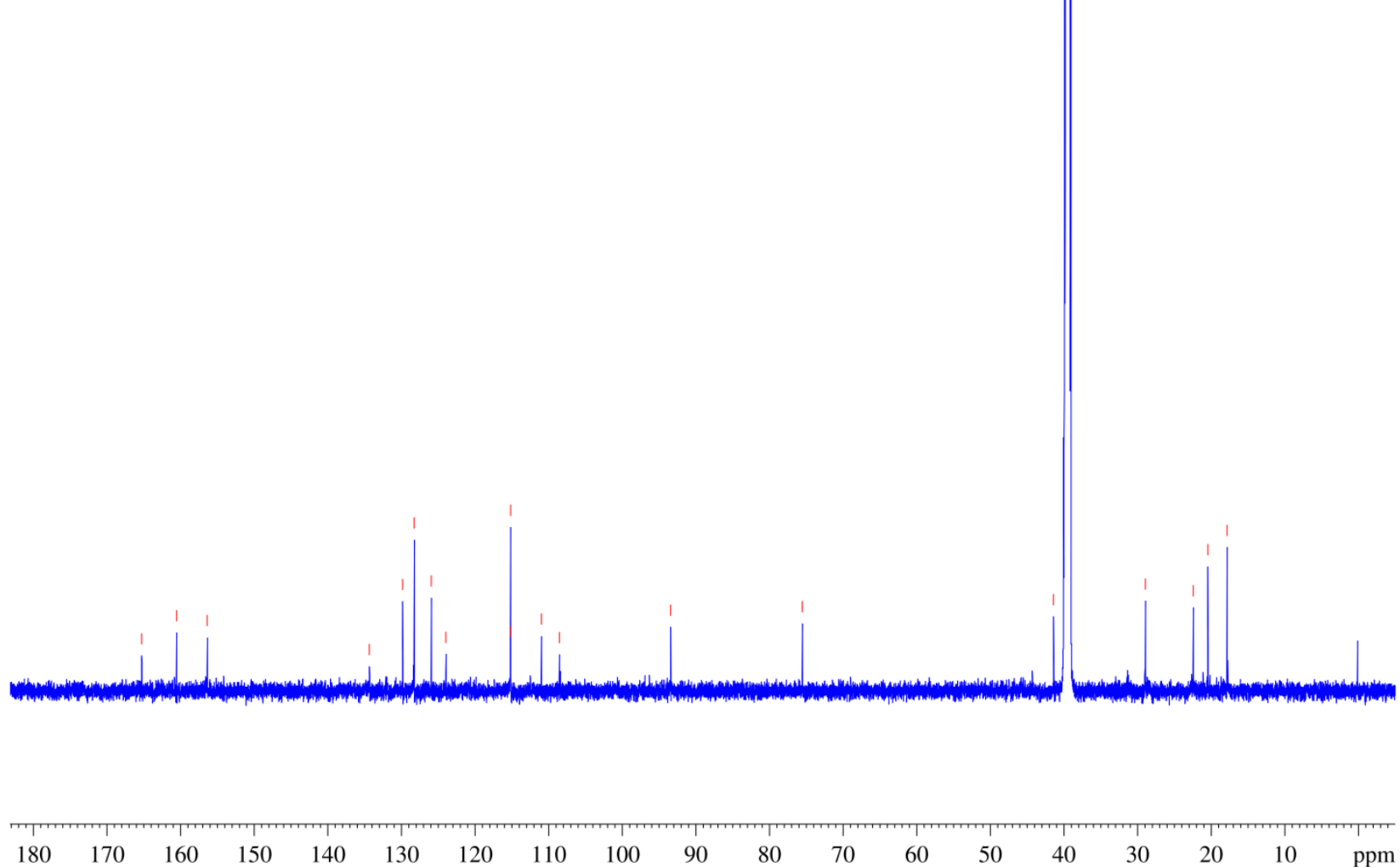

Fig. S37 HSQC spectrum of compound 8

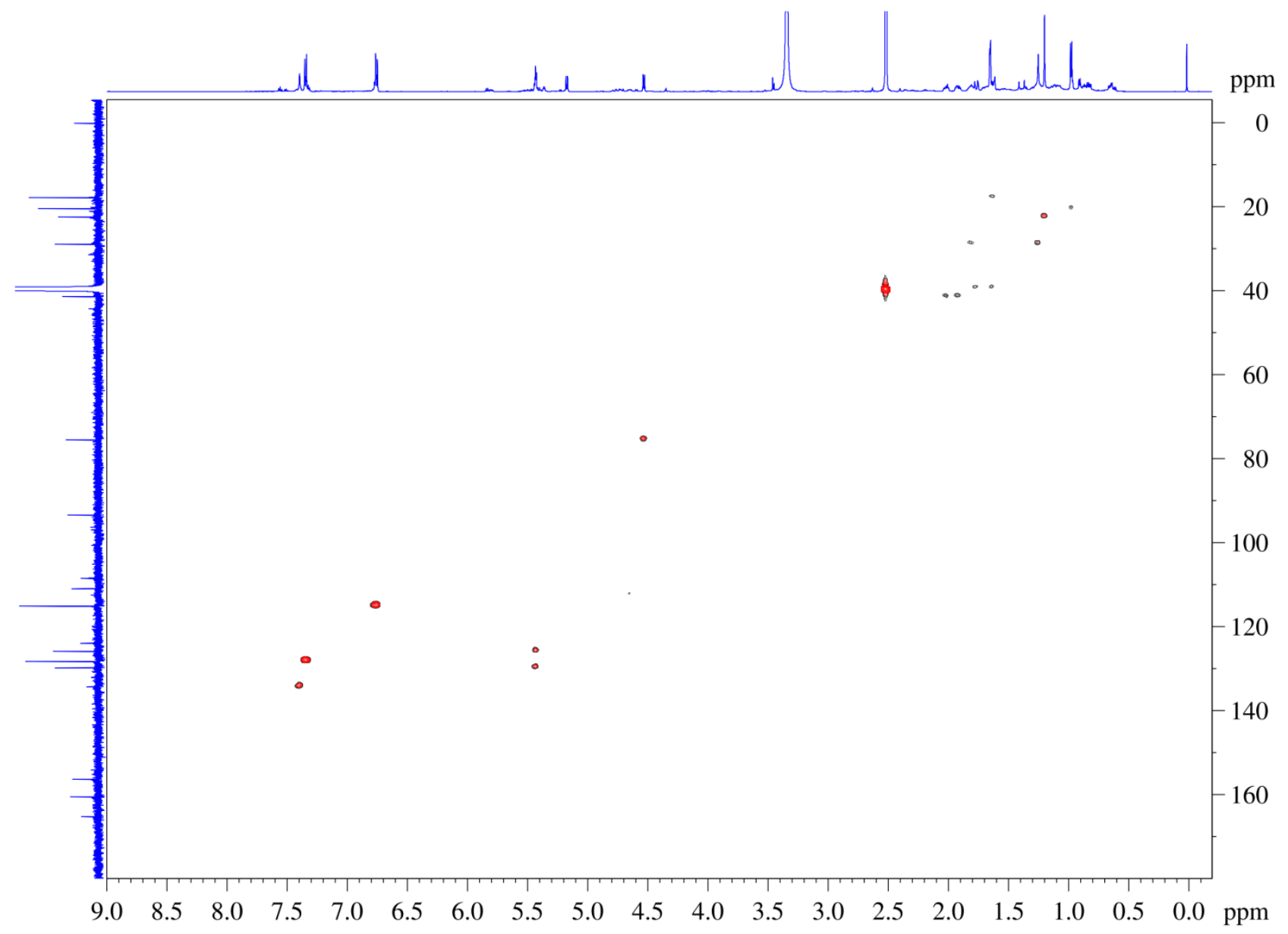


Fig. S38 HMBC spectrum of compound 8

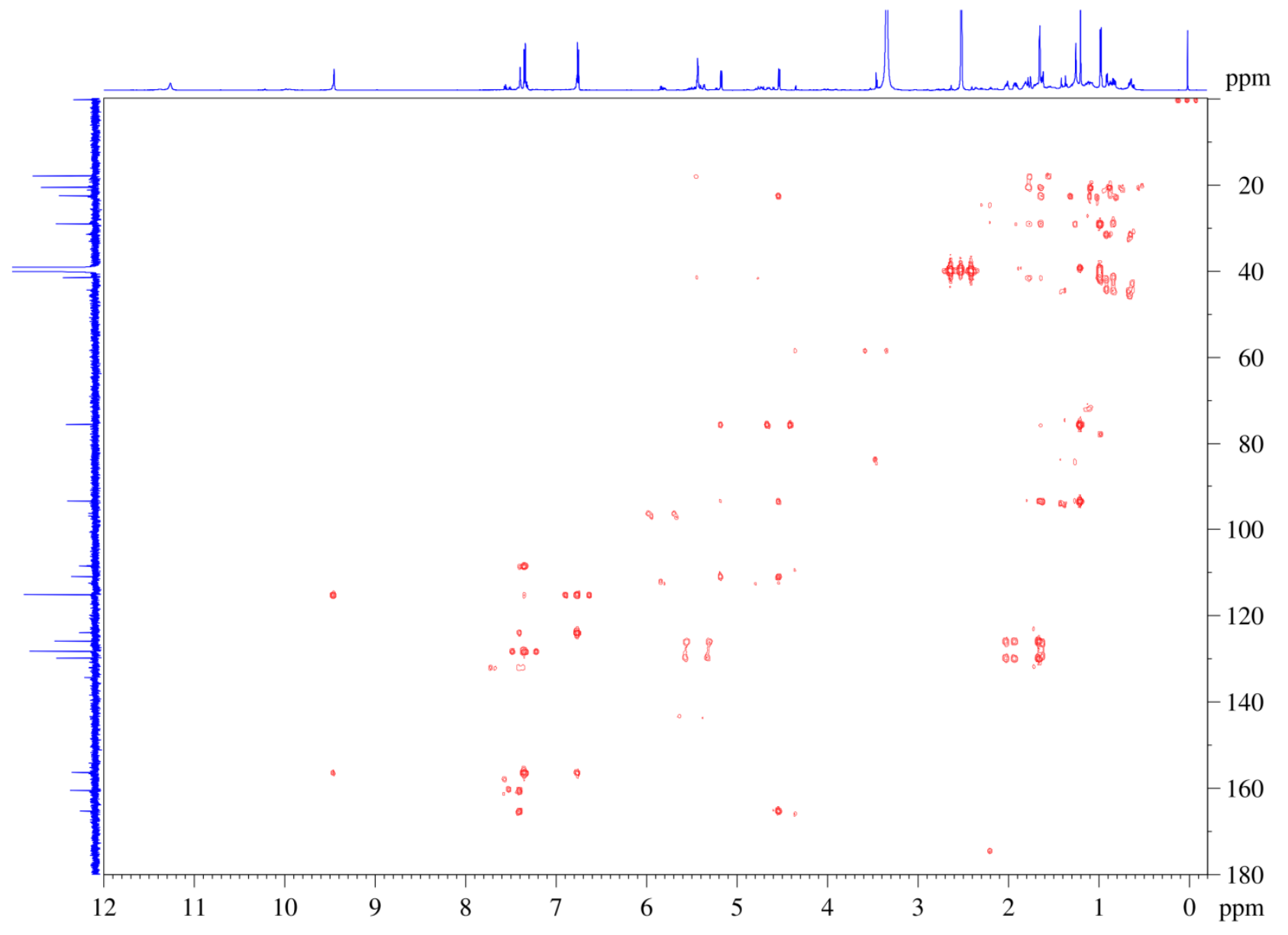

Fig. S39 ${ }^{1} \mathrm{H}-{ }^{1} \mathrm{H}$ COSY spectrum of compound 8

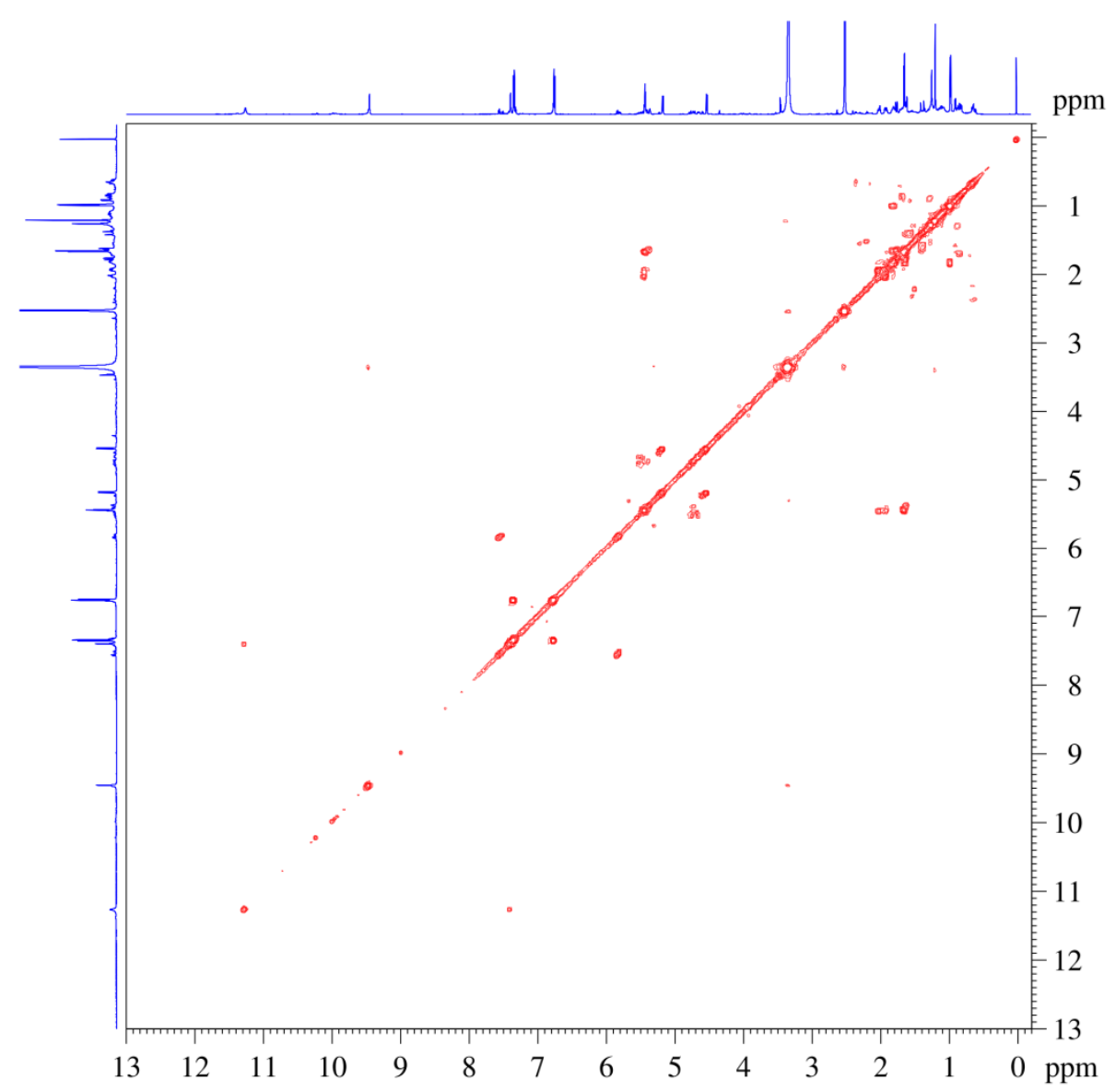


Fig. S40 HRESIMS report of compound 3

\section{Elemental Composition Report}

\section{Single Mass Analysis}

Tolerance $=50.0 \mathrm{mDa} \quad / \quad \mathrm{DBE}: \min =-1.5, \max =50.0$

Element prediction: Off

Number of isotope peaks used for i-FIT $=3$

Monoisotopic Mass, Even Electron Ions

22 formula(e) evaluated with 5 results within limits (up to 50 best isotopic matches for each mass)

Elements Used:
$\begin{array}{llll}\mathrm{C}: 0-500 & \mathrm{H}: 0-1000 & \mathrm{~N}: 1-1 & \mathrm{O}: 1-5\end{array}$

$3 \mathrm{G} 6$

20191223-3 219 (1.773)

1: TOF MS ES+

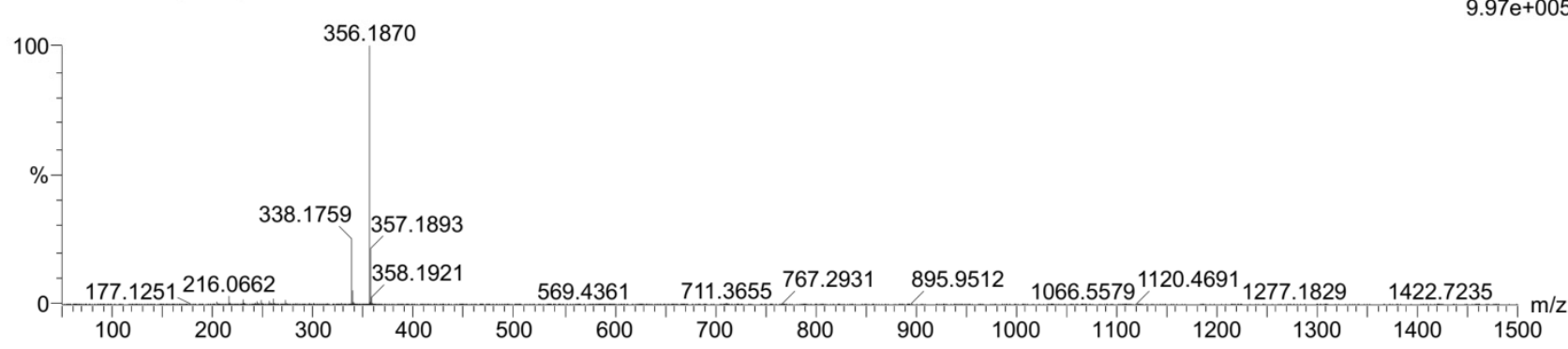

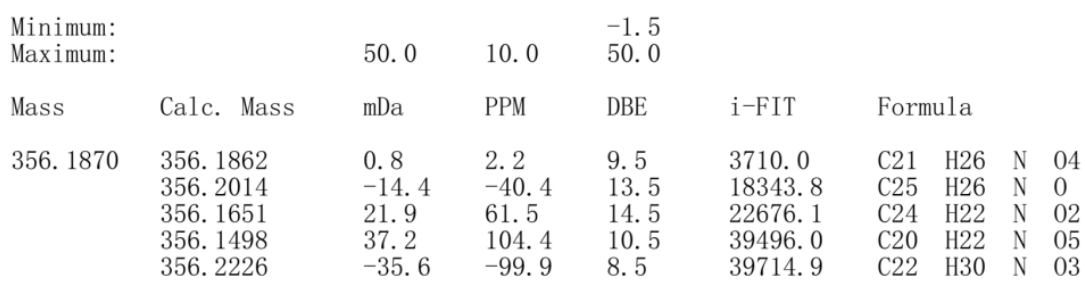


Fig. S41 HRESIMS report of compound 4

\section{Elemental Composition Report}

\section{Single Mass Analysis}

Tolerance $=50.0 \mathrm{mDa} / \mathrm{DBE}: \min =-1.5, \max =50.0$

Element prediction: Off

Number of isotope peaks used for i-FIT $=3$

Monoisotopic Mass, Even Electron lons

17 formula(e) evaluated with 5 results within limits (up to 50 best isotopic matches for each mass)

Elements Used:
$\begin{array}{llll}\mathrm{C}: 0-500 & \mathrm{H}: 0-1000 & \mathrm{~N}: 1-1 & \mathrm{O}: 1-5\end{array}$

$3 \mathrm{G} 5$

20191223-4 $146(1.182)$

1: TOF MS ES+

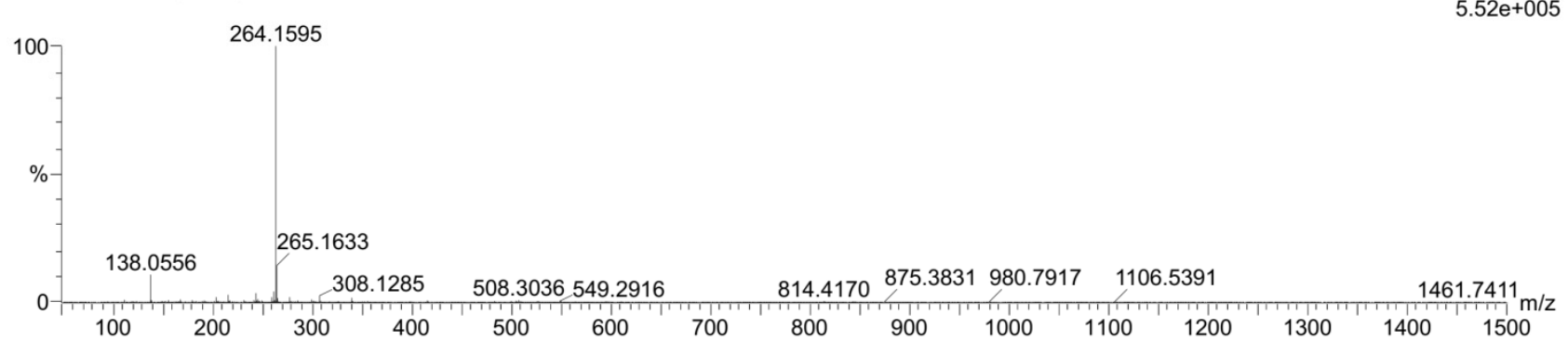

\begin{tabular}{|c|c|c|c|c|c|c|c|}
\hline $\begin{array}{l}\text { Minimum: } \\
\text { Maximum: }\end{array}$ & & 50.0 & 10.0 & $\begin{array}{l}-1.5 \\
50.0\end{array}$ & & & \\
\hline Mass & Calc. Mass & $\mathrm{mDa}$ & PPM & DBE & i-FIT & Formula & \\
\hline 264. 1595 & $\begin{array}{l}264.1600 \\
264.1811 \\
264.1388 \\
264.1236 \\
264.1964\end{array}$ & $\begin{array}{l}-0.5 \\
-21.6 \\
20.7 \\
35.9 \\
-36.9\end{array}$ & $\begin{array}{l}-1.9 \\
-81.8 \\
78.4 \\
135.9 \\
-139.7\end{array}$ & $\begin{array}{l}5.5 \\
0.5 \\
10.5 \\
6.5 \\
4.5\end{array}$ & $\begin{array}{l}2601.4 \\
12362.2 \\
18477.7 \\
34373.9 \\
38286.2\end{array}$ & $\begin{array}{ll}\mathrm{C} 15 & \mathrm{H} 22 \\
\mathrm{C} 12 & \mathrm{H} 26 \\
\mathrm{C} 18 & \mathrm{H} 18 \\
\mathrm{C} 14 & \mathrm{H} 18 \\
\mathrm{C} 16 & \mathrm{H} 26\end{array}$ & $\begin{array}{ll}\mathrm{N} & 03 \\
\mathrm{~N} & 05 \\
\mathrm{~N} & 0 \\
\mathrm{~N} & 04 \\
\mathrm{~N} & 02\end{array}$ \\
\hline
\end{tabular}


Fig. S42 HRESIMS report of compound 5

Elemental Composition Report

Page 1

\section{Single Mass Analysis}

Tolerance $=50.0 \mathrm{mDa} /$ DBE: $\min =-1.5, \max =50.0$

Element prediction: Off

Number of isotope peaks used for i-FIT $=3$

Monoisotopic Mass, Even Electron lons

380 formula(e) evaluated with 32 results within limits (up to 50 best isotopic matches for each mass)

Elements Used:

$\begin{array}{llll}\text { C: } 0-500 & H: 0-1000 & \text { N: } 0-5 & \text { O: } 0-200\end{array}$

60-65-6

$20191118032159(1.290)$

1: TOF MS ES+

$9.59 \mathrm{e}+005$

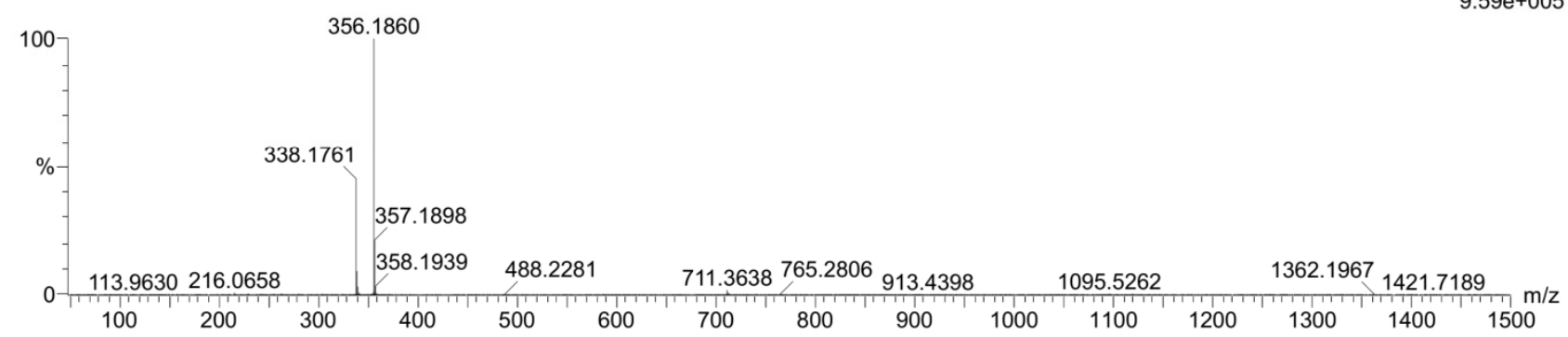

\begin{tabular}{|c|c|c|c|c|c|c|c|c|c|}
\hline \multicolumn{2}{|l|}{$\begin{array}{l}\text { Minimum: } \\
\text { Maximum: }\end{array}$} & 50.0 & 10.0 & $\begin{array}{l}-1.5 \\
50.0\end{array}$ & & & & & \\
\hline Mass & Calc. Mass & $\mathrm{mDa}$ & PPM & DBE & i-FIT & Form & ula & & \\
\hline 356. 1860 & $\begin{array}{l}356.1862 \\
356.1875 \\
356.1822 \\
356.1921 \\
356.1934 \\
356.1781 \\
356.1763 \\
356.1974 \\
356.1723 \\
356.1709 \\
356.2014 \\
356.2033 \\
356.1669 \\
356.1651 \\
356.2073 \\
356.2087 \\
356.1610 \\
356.2127 \\
356.2145 \\
356.1570 \\
356.1557 \\
356.2185 \\
356.1511 \\
356.1498 \\
356.2226 \\
356.1458 \\
356.1439 \\
356.2284 \\
356.2298 \\
356.1418 \\
356.1399 \\
356.2338\end{array}$ & $\begin{array}{l}-0.2 \\
-1.5 \\
3.8 \\
-6.1 \\
-7.4 \\
7.9 \\
9.7 \\
-11.4 \\
13.7 \\
15.1 \\
-15.4 \\
-17.3 \\
19.1 \\
20.9 \\
-21.3 \\
-22.7 \\
25.0 \\
-26.7 \\
-28.5 \\
29.0 \\
30.3 \\
-32.5 \\
34.9 \\
36.2 \\
-36.6 \\
40.2 \\
42.1 \\
-42.4 \\
-43.8 \\
44.2 \\
46.1 \\
-47.8\end{array}$ & $\begin{array}{l}-0.6 \\
-4.2 \\
10.7 \\
-17.1 \\
-20.8 \\
22.2 \\
27.2 \\
-32.0 \\
38.5 \\
42.4 \\
-43.2 \\
-48.6 \\
53.6 \\
58.7 \\
-59.8 \\
-63.7 \\
70.2 \\
-75.0 \\
-80.0 \\
81.4 \\
85.1 \\
-91.2 \\
98.0 \\
101.6 \\
-102.8 \\
112.9 \\
118.2 \\
-119.0 \\
-123.0 \\
124.1 \\
129.4 \\
-134.2\end{array}$ & $\begin{array}{l}9.5 \\
14.5 \\
5.5 \\
0.5 \\
5.5 \\
1.5 \\
14.5 \\
9.5 \\
10.5 \\
5.5 \\
13.5 \\
0.5 \\
1.5 \\
14.5 \\
4.5 \\
9.5 \\
10.5 \\
13.5 \\
0.5 \\
6.5 \\
1.5 \\
4.5 \\
15.5 \\
10.5 \\
8.5 \\
6.5 \\
19.5 \\
-0.5 \\
4.5 \\
2.5 \\
15.5 \\
8.5\end{array}$ & $\begin{array}{l}\text { 1842.8 } \\
5179.2 \\
990.0 \\
4801.7 \\
2458.5 \\
10474.7 \\
8522.7 \\
4586.3 \\
5431.9 \\
6348.7 \\
15682.0 \\
12437.3 \\
17078.5 \\
18709.0 \\
11678.6 \\
14097.7 \\
16953.2 \\
26659.9 \\
26831.6 \\
24540.4 \\
30426.2 \\
27312.5 \\
35380.5 \\
35177.4 \\
37908.6 \\
44037.9 \\
61759.4 \\
48811.3 \\
49896.1 \\
63641.9 \\
59763.2 \\
61663.6\end{array}$ & $\begin{array}{l}\text { C21 } \\
\text { C22 } \\
\text { C16 } \\
\text { C14 } \\
\text { C15 } \\
\text { C11 } \\
\text { C23 } \\
\text { C20 } \\
\text { C18 } \\
\text { C17 } \\
\text { C25 } \\
\text { C13 } \\
\text { C12 } \\
\text { C24 } \\
\text { C18 } \\
\text { C19 } \\
\text { C19 } \\
\text { C24 } \\
\text { C12 } \\
\text { C14 } \\
\text { C13 } \\
\text { C17 } \\
\text { C21 } \\
\text { C20 } \\
\text { C22 } \\
\text { C15 } \\
\text { C27 } \\
\text { C15 } \\
\text { C16 } \\
\text { C10 } \\
\text { C22 } \\
\text { C21 }\end{array}$ & $\begin{array}{l}\text { H26 } \\
\text { H22 } \\
\text { H26 } \\
\text { H30 } \\
\text { H26 } \\
\text { H26 } \\
\text { H22 } \\
\text { H26 } \\
\text { H22 } \\
\text { H26 } \\
\text { H26 } \\
\text { H30 } \\
\text { H26 } \\
\text { H22 } \\
\text { H30 } \\
\text { H26 } \\
\text { H22 } \\
\text { H26 } \\
\text { H30 } \\
\text { H22 } \\
\text { H26 } \\
\text { H30 } \\
\text { H18 } \\
\text { H22 } \\
\text { H30 } \\
\text { H22 } \\
\text { H18 } \\
\text { H34 } \\
\text { H30 } \\
\text { H22 } \\
\text { H18 } \\
\text { H30 }\end{array}$ & $\begin{array}{l}\text { N } \\
\text { N5 } \\
\text { N3 } \\
\text { N } \\
\text { N5 } \\
\text { N5 } \\
\text { N3 } \\
\text { N3 } \\
\text { N5 } \\
\text { N } \\
\text { N } \\
\text { N3 } \\
\text { N3 } \\
\text { N } \\
\text { N } \\
\text { N5 } \\
\text { N3 } \\
\text { N3 } \\
\text { N5 } \\
\text { N5 } \\
\text { N } \\
\text { N3 } \\
\text { N5 } \\
\text { N } \\
\text { N } \\
\text { N3 } \\
\text { N } \\
\text { N } \\
\text { N5 } \\
\text { N5 } \\
\text { N3 } \\
\text { N3 }\end{array}$ & $\begin{array}{c}04 \\
06 \\
09 \\
05 \\
08 \\
0 \\
03 \\
03 \\
07 \\
0 \\
08 \\
09 \\
02 \\
06 \\
02\end{array}$ \\
\hline
\end{tabular}


Fig. S43 HRESIMS report of compound 6

Elemental Composition Report

\section{Single Mass Analysis}

Tolerance $=50.0 \mathrm{mDa} / \mathrm{DBE}: \min =-1.5, \max =50.0$

Element prediction: Off

Number of isotope peaks used for i-FIT $=3$

Monoisotopic Mass, Even Electron Ions

380 formula(e) evaluated with 32 results within limits (up to 50 best isotopic matches for each mass)

Elements Used:

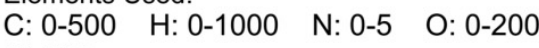

60-65-7

20190819-06 179 (1.451)

1: TOF MS ES+

$1.47 \mathrm{e}+006$

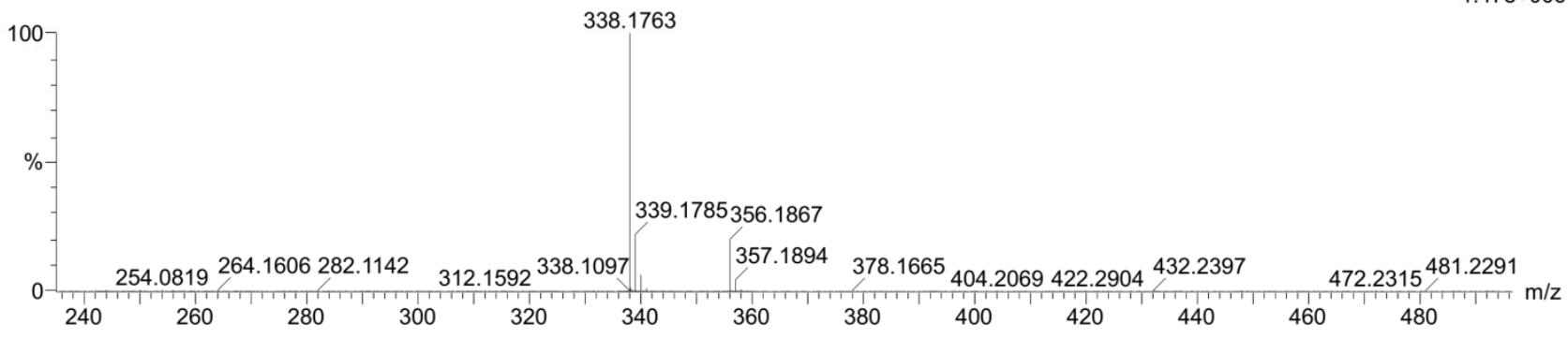

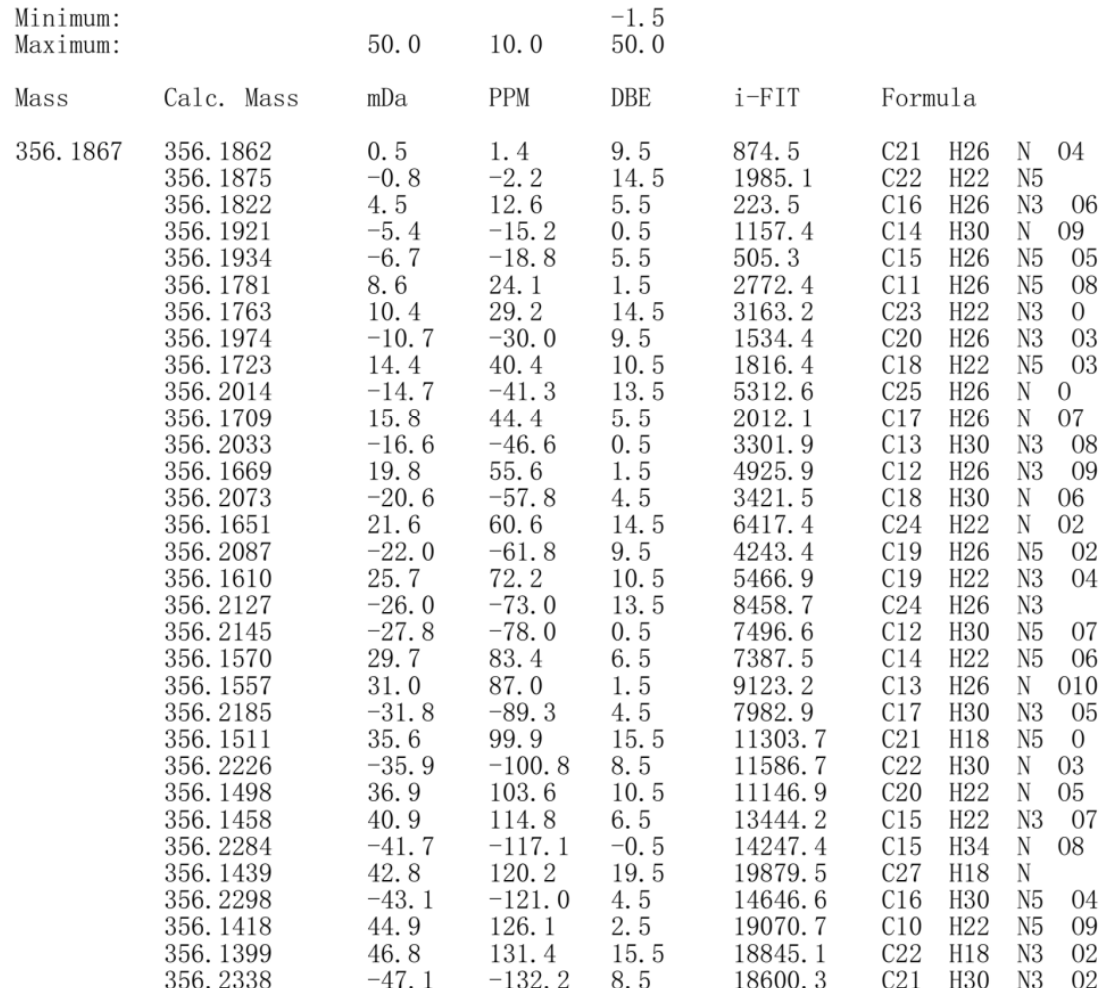


Fig. S44 HRESIMS report of compound 7

Elemental Composition Report

Page 1

\section{Single Mass Analysis}

Tolerance $=50.0 \mathrm{mDa} / \mathrm{DBE}: \min =-1.5, \max =50.0$

Element prediction: Off

Number of isotope peaks used for i-FIT $=3$

Monoisotopic Mass, Even Electron lons

380 formula(e) evaluated with 32 results within limits (up to 50 best isotopic matches for each mass)

Elements Used:

$\begin{array}{llll}\text { C: } 0-500 & \mathrm{H}: 0-1000 & \mathrm{~N}: 0-5 & \mathrm{O}: 0-200 \\ 60-65-8 & & \end{array}$

20191118033176 (1.429)

1: TOF MS ES+

$1.89 \mathrm{e}+006$

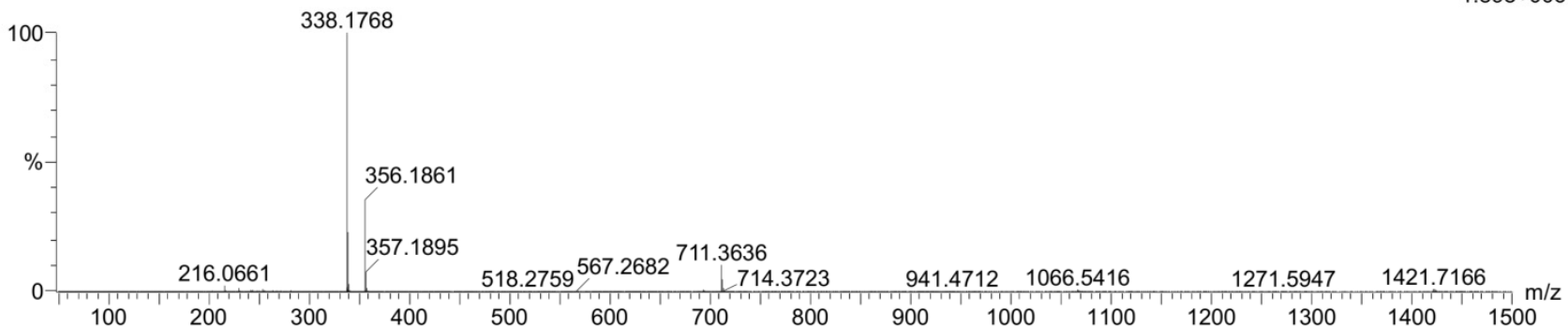

\begin{tabular}{|c|c|c|c|c|c|c|c|c|c|}
\hline $\begin{array}{l}\text { Minimum: } \\
\text { Maximum: }\end{array}$ & & 50.0 & 10.0 & $\begin{array}{l}-1.5 \\
50.0\end{array}$ & & & & & \\
\hline Mass & Calc. Mass & $\mathrm{mDa}$ & PPM & DBE & i-FIT & Form & גa & & \\
\hline 356. 1861 & $\begin{array}{l}356.1862 \\
356.1875 \\
356.1822 \\
356.1921 \\
356.1934 \\
356.1781 \\
356.1763 \\
356.1974 \\
356.1723 \\
356.1709 \\
356.2014 \\
356.2033 \\
356.1669 \\
356.1651 \\
356.2073 \\
356.2087 \\
356.1610 \\
356.2127 \\
356.2145 \\
356.1570 \\
356.1557 \\
356.2185 \\
356.1511 \\
356.1498 \\
356.2226 \\
356.1458 \\
356.1439 \\
356.2284 \\
356.2298 \\
356.1418 \\
356.1399 \\
356.2338\end{array}$ & $\begin{array}{l}-0.1 \\
-1.4 \\
3.9 \\
-6.0 \\
-7.3 \\
8.0 \\
9.8 \\
-11.3 \\
13.8 \\
15.2 \\
-15.3 \\
-17.2 \\
19.2 \\
21.0 \\
-21.2 \\
-22.6 \\
25.1 \\
-26.6 \\
-28.4 \\
29.1 \\
30.4 \\
-32.4 \\
35.0 \\
36.3 \\
-36.5 \\
40.3 \\
42.2 \\
-42.3 \\
-43.7 \\
44.3 \\
46.2 \\
-47.7\end{array}$ & $\begin{array}{l}-0.3 \\
-3.9 \\
10.9 \\
-16.8 \\
-20.5 \\
22.5 \\
27.5 \\
-31.7 \\
38.7 \\
42.7 \\
-43.0 \\
-48.3 \\
53.9 \\
59.0 \\
-59.5 \\
-63.4 \\
70.5 \\
-74.7 \\
-79.7 \\
81.7 \\
85.3 \\
-91.0 \\
98.3 \\
101.9 \\
-102.5 \\
113.1 \\
118.5 \\
-118.8 \\
-122.7 \\
124.4 \\
129.7 \\
-133.9\end{array}$ & $\begin{array}{l}9.5 \\
14.5 \\
5.5 \\
0.5 \\
5.5 \\
1.5 \\
14.5 \\
9.5 \\
10.5 \\
5.5 \\
13.5 \\
0.5 \\
1.5 \\
14.5 \\
4.5 \\
9.5 \\
10.5 \\
13.5 \\
0.5 \\
6.5 \\
1.5 \\
4.5 \\
15.5 \\
10.5 \\
8.5 \\
6.5 \\
19.5 \\
-0.5 \\
4.5 \\
2.5 \\
15.5 \\
8.5\end{array}$ & $\begin{array}{l}\text { 1448. } 7 \\
3949.6 \\
619.0 \\
3040.6 \\
1626.1 \\
6915.8 \\
6249.4 \\
3342.3 \\
3891.8 \\
4315.6 \\
11200.7 \\
8298.5 \\
11472.1 \\
13266.2 \\
8020.7 \\
9893.1 \\
11835.9 \\
18772.7 \\
18213.9 \\
16814.9 \\
20673.2 \\
18795.3 \\
24736.1 \\
24394.6 \\
26334.3 \\
30256.8 \\
43101.8 \\
33399.1 \\
34352.3 \\
43530.7 \\
41540.3 \\
42708.7\end{array}$ & $\begin{array}{l}\text { C21 } \\
\text { C22 } \\
\text { C16 } \\
\text { C14 } \\
\text { C15 } \\
\text { C11 } \\
\text { C23 } \\
\text { C20 } \\
\text { C18 } \\
\text { C17 } \\
\text { C25 } \\
\text { C13 } \\
\text { C12 } \\
\text { C24 } \\
\text { C18 } \\
\text { C19 } \\
\text { C19 } \\
\text { C24 } \\
\text { C12 } \\
\text { C14 } \\
\text { C13 } \\
\text { C17 } \\
\text { C21 } \\
\text { C20 } \\
\text { C22 } \\
\text { C15 } \\
\text { C27 } \\
\text { C15 } \\
\text { C16 } \\
\text { C10 } \\
\text { C22 } \\
\text { C21 }\end{array}$ & $\begin{array}{l}\text { H26 } \\
\text { H22 } \\
\text { H26 } \\
\text { H30 } \\
\text { H26 } \\
\text { H26 } \\
\text { H22 } \\
\text { H26 } \\
\text { H22 } \\
\text { H26 } \\
\text { H26 } \\
\text { H30 } \\
\text { H26 } \\
\text { H22 } \\
\text { H30 } \\
\text { H26 } \\
\text { H22 } \\
\text { H26 } \\
\text { H30 } \\
\text { H22 } \\
\text { H26 } \\
\text { H30 } \\
\text { H18 } \\
\text { H22 } \\
\text { H30 } \\
\text { H22 } \\
\text { H18 } \\
\text { H34 } \\
\text { H30 } \\
\text { H22 } \\
\text { H18 } \\
\text { H30 }\end{array}$ & $\begin{array}{l}\text { N } \\
\text { N5 } \\
\text { N3 } \\
\text { N } \\
\text { N5 } \\
\text { N5 } \\
\text { N3 } \\
\text { N3 } \\
\text { N5 } \\
\text { N } \\
\text { N } \\
\text { N3 } \\
\text { N3 } \\
\text { N } \\
\text { N } \\
\text { N5 } \\
\text { N3 } \\
\text { N3 } \\
\text { N5 } \\
\text { N5 } \\
\text { N } \\
\text { N3 } \\
\text { N5 } \\
\text { N } \\
\text { N } \\
\text { N3 } \\
\text { N } \\
\text { N } \\
\text { N5 } \\
\text { N5 } \\
\text { N3 } \\
\text { N3 }\end{array}$ & $\begin{array}{c}04 \\
06 \\
09 \\
05 \\
08 \\
0 \\
03 \\
03 \\
07 \\
0 \\
08 \\
09 \\
02 \\
06 \\
02 \\
04\end{array}$ \\
\hline
\end{tabular}


Fig. S45 HRESIMS report of compound 8

Elemental Composition Report

Page 1

\section{Single Mass Analysis}

Tolerance $=50.0 \mathrm{mDa} / \mathrm{DBE}: \min =-1.5, \max =50.0$

Element prediction: Off

Number of isotope peaks used for i-FIT $=3$

Monoisotopic Mass, Even Electron Ions

380 formula(e) evaluated with 32 results within limits (up to 50 best isotopic matches for each mass)

Elements Used:
C: $0-500 \quad \mathrm{H}: 0-1000$
$\mathrm{N}: 0-5 \quad$ O: $0-200$
60-65-9

$20191118034178(1.444)$

1: TOF MS ES+

$1.58 \mathrm{e}+006$

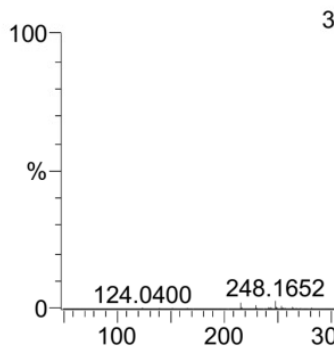

338.1766

339.1796

\begin{tabular}{|llllllll}
357.1895 & 532.2560 & 711.3638 & 817.4628 & 941.4783 & 1066.5442 & 1296.6407 & 1421.7189 \\
\hline
\end{tabular}

Minimum:

Maximum:

Mass

356. 1861

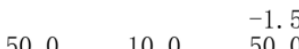

$\mathrm{mDa} \quad \mathrm{PPM} \quad \mathrm{DBE}$

$\begin{array}{lll}-0.1 & -0.3 & 9 .\end{array}$

$\begin{array}{lll}3.9 & 10.9 & 5.5\end{array}$

$\begin{array}{lll}3.9 & -20.5 & 5.5\end{array}$

$\begin{array}{lll}-11.3 & -31.7 & 9.5\end{array}$

$\begin{array}{lll}-6.0 & -16.8 & 0.5\end{array}$

$\begin{array}{lll}15.2 & 42.7 & 5.5\end{array}$

$\begin{array}{lll}13.8 & 38.7 & 10.5\end{array}$

$9.8 \quad 27.5 \quad 14.5$

$\begin{array}{lll}-21.2 & -59.5 & 4.5\end{array}$

$\begin{array}{lll}8.0 & 22.5 & 1.5 \\ -15.3 & -43.0 & 13.5\end{array}$

$\begin{array}{lll}-15.3 & -43.0 & 13.5 \\ -17.2 & -48.3 & 0.5\end{array}$

$\begin{array}{lll}-22.6 & -63.4 & 9.5\end{array}$

$\begin{array}{lll}21.0 & 59.0 & 14.5\end{array}$

$\begin{array}{lll}25.1 & 70.5 & 10.5\end{array}$

19. $2 \quad 53.9 \quad 1.5$

$-26.6 \quad-74.7 \quad 13.5$

$\begin{array}{lll}29.1 & 81.7 & 6.5\end{array}$

$-32.4 \quad-91.0 \quad 4.5$

$-28.4 \quad-79.7 \quad 0.5$

$30.4 \quad 85.3 \quad 1.5$

$36.3 \quad 101.9 \quad 10.5$

$\begin{array}{lll}35.0 & 98.3 & 15.5\end{array}$

$-36.5-102.5 \quad 8.5$

$\begin{array}{lll}40.3 & 113.1 & 6.5\end{array}$

$-42.3 \quad-118.8 \quad-0.5$

$-43.7 \quad-122.7 \quad 4.5$

$\begin{array}{lll}42.2 & 118.5 & 19.5\end{array}$

46. $2 \quad 129.7 \quad 15.5$

$\begin{array}{lll}-47.7 & -133.9 & 8.5\end{array}$

$\begin{array}{lll}44.3 & 124.4 & 2.5\end{array}$
i-FIT

Formula

1067. 4

2006.9 C15 H26 N5 05

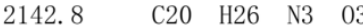

2288. $6 \quad$ C22 H22 N5

2315. $3 \quad \mathrm{C} 14 \quad \mathrm{H} 30$ N 09

$\begin{array}{lllll}2679.6 & \mathrm{C} 17 & \mathrm{H} 26 & \mathrm{~N} & 07\end{array}$

2731. 0 C18 H22 N5 03

$\begin{array}{lllll}3000.8 & \mathrm{C} 23 & \mathrm{H} 22 & \mathrm{~N} 3 & 0\end{array}$

4179. $1 \quad \mathrm{C} 18$ H30 N 06

4442. $4 \quad$ C11 H26 N5 08

4677.1 $\quad \mathrm{C} 25 \quad \mathrm{H} 26 \quad \mathrm{~N} \quad 0$

$\begin{array}{lllll}4786.5 & \text { C13 } & \text { H30 } & \text { N3 } & 08\end{array}$

5180.9 $\quad$ C19 H26 N5 02

$5701.9 \quad \mathrm{C} 24 \quad \mathrm{H} 22$ N 02

5870. $4 \quad$ C19 H22 N3 04

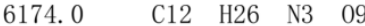

8194. 0 C24 H26 N3

8548.0 C14 H22 N5 06

$\begin{array}{lllll}9017.5 & \mathrm{C} 17 & \mathrm{H} 30 & \mathrm{~N} 3 & 05\end{array}$

$\begin{array}{lllll}9220.5 & \mathrm{C} 12 & \mathrm{H} 30 & \mathrm{~N} 5 & 07\end{array}$

9864. $3 \quad \mathrm{C} 13$ H26 N 010

$\begin{array}{lllll}10955.7 & \mathrm{C} 20 & \mathrm{H} 22 & \mathrm{~N} & 05\end{array}$

11280.0 C21 H18 N5 0

$11553.9 \quad \mathrm{C} 22 \quad \mathrm{H} 30 \quad \mathrm{~N} \quad 03$

14050.2 $\quad$ C15 $\mathrm{H} 22 \quad \mathrm{~N} 3 \quad 07$

15205.3 C15 H34 N 08

15871.8 C16 H30 N5 04

17929.4 C27 H18 N

18174. $2 \quad$ C22 H18 N3 02

18799. $4 \quad \mathrm{C} 21 \quad \mathrm{H} 30 \quad \mathrm{~N} 3 \quad 02$

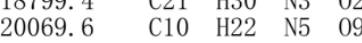


Fig. S46 HPLC-DAD-MS analysis of Tolypocladium sp. 49Y metabolites in different media
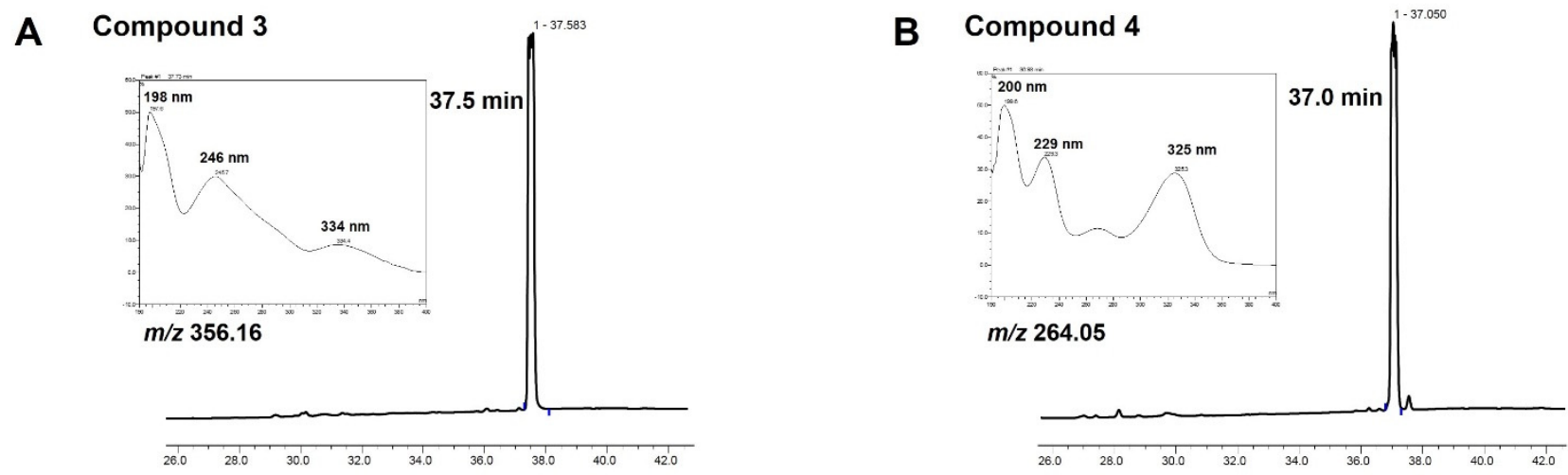

\section{Tolypocladium sp. $49 \mathrm{Y}$ rice ferment $60 \mathrm{~d}$}

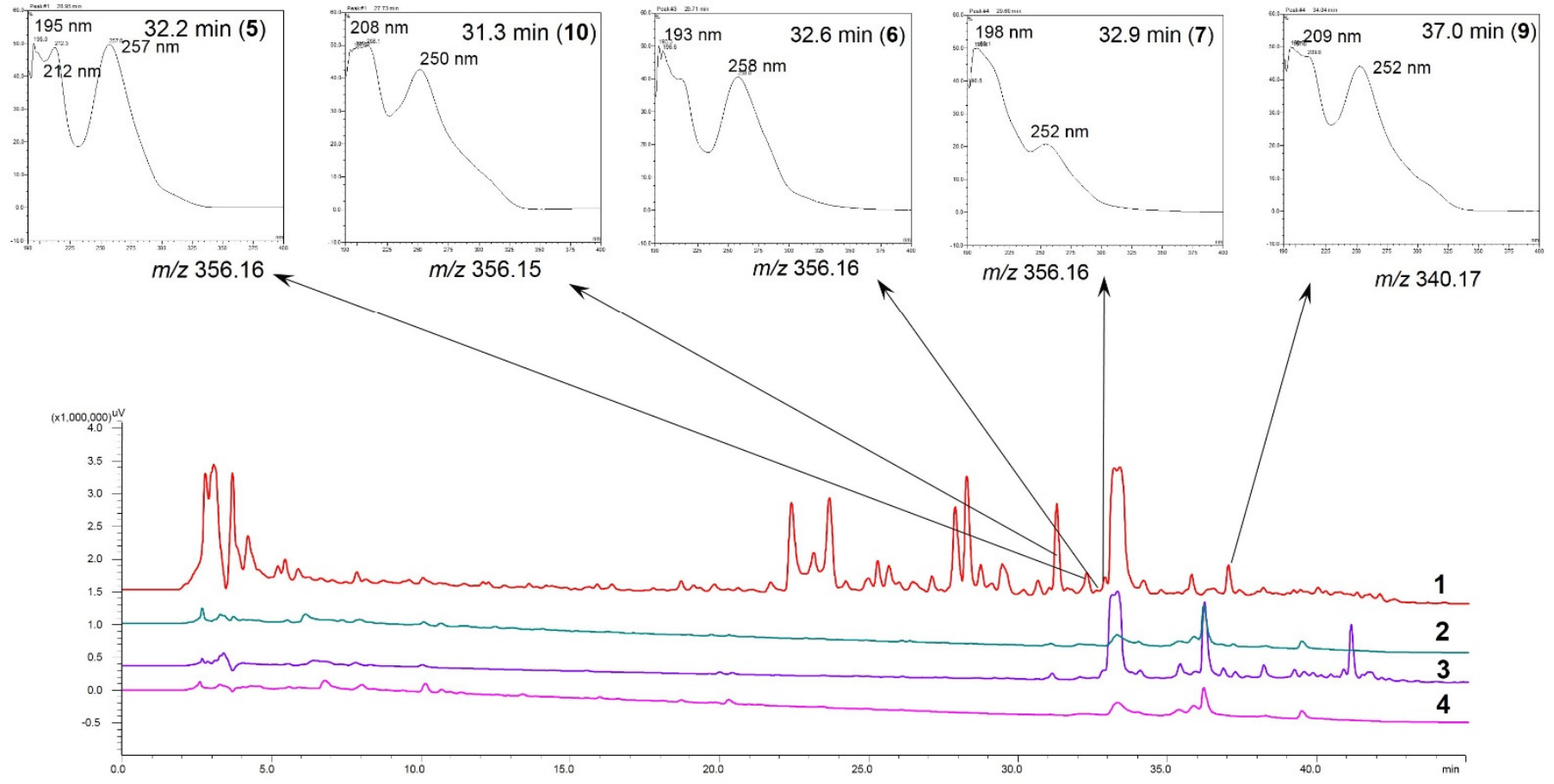

Flow rate: $1 \mathrm{~mL} / \mathrm{min}$; wavelength: $280 \mathrm{~nm}$; time/B\% (methanol): 0-40 $\mathrm{min} / 20-90 \%, 40-45 \mathrm{~min} / 90 \%-100 \%$; A: HPLC-DAD-MS analysis of compound 3; B: HPLC-DAD-MS analysis of compound 4; C: HPLC-DADMS analysis of Tolypocladium sp. 49Y metabolites in different media; 1: rice ferments; 2: XC-3 medium; 3 : potato dextrose broth (PDB); 4: maltose medium. All above shown chromatographs are detected at wavelength of $280 \mathrm{~nm}$. 
Fig. S47 Phylogenetic analysis of PKS-NRPSs encoded by four hit genes (PKS-NRPS) from Tolypocladium sp. 49Y

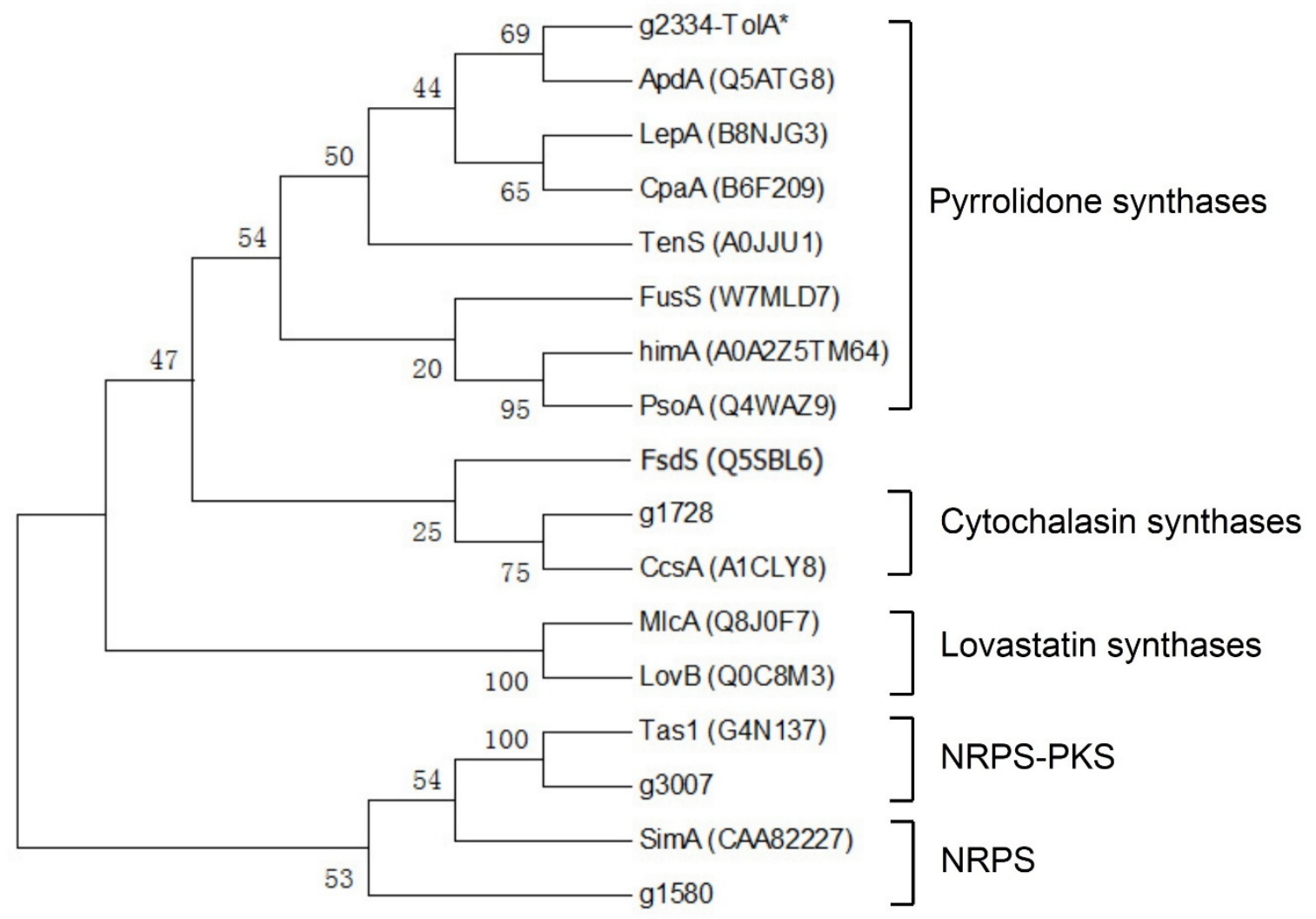

Phylogenic analysis was conducted by Maximum-likelihood method. Hit genes in Tolypyridone sp. 49Y are g2334 (Genbank accession number: MT127616), g1728, g1580 and g3007. Swiss-prot accession numbers of all included proteins are given in the brackets. 


\section{Reference}

(1) Fujii, T.; Yamaoka, H.; Gomi, K.; Kitamoto, A.; Kumagai, C., Cloning and nucleotide sequence of the ribonuclease T1 gene (rntA) from Aspergillus oryzae and its expression in Saccharomyces cerevisiae and Aspergillus oryzae. Biosci. Biotechnol. Biochem. 1995, 59 (10), 1869-1874.

(2) Yamada, O.; Nan, S. N.; Akao, T.; Tominaga, M.; Watanabe, H.; Satoh, T.; Enei, H.; Akita, O., dffA Gene fromAspergillus oryzae encodesl-ornithineN5-oxygenase and is indispensable for deferriferrichrysin biosynthesis. J. Biosci. Bioeng. 2003, 95 (1), 82-88.

(3) Jin, F. J.; Maruyama, J.; Juvvadi, P. R.; Arioka, M.; Kitamoto, K., Adenine auxotrophic mutants of Aspergillus oryzae: development of a novel transformation system with triple auxotrophic hosts. Biosci. Biotechnol. Biochem. 2004, 68 (3), 656-62.

(4) Jin, F. J.; Maruyama, J.-i.; Juvvadi, P. R.; Arioka, M.; Kitamoto, K., Development of a novel quadruple auxotrophic host transformation system by $\arg B$ gene disruption using adeA gene and exploiting adenine auxotrophy in Aspergillus oryzae. FEMS Microbiol. Lett. 2004, 239 (1), 79-85.

(5) Xu, Y.; Wang, L.; Zhu, G.; Zuo, M.; Gong, Q.; He, W.; Li, M.; Yuan, C.; Hao, X.; Zhu, W., New phenylpyridone derivatives from the Penicillium sumatrense GZWMJZ-313, a fungal endophyte of Garcinia multiflora. Chin. Chem. Lett. 2019, 30 (2), 431-434.

(6) (a) Fukuda, T.; Sudoh, Y.; Tsuchiya, Y.; Okuda, T.; Matsuura, N.; Motojima, A.; Oikawa, T.; Igarashi, Y., Tolypoalbin, a new tetramic acid from Tolypocladium album TAMA 479. J. Antibiot. 2015, 68 (6), 399; (b) Robert M. Silverstein, F. X. W., David J. Kiemle, Chapter 4. Carbon-13 NMR spectrometry. In Spectroscopic Identification of Organic Compounds, John Wiley\&Sons: 2005.

(7) Frisch, M.; Trucks, G.; Schlegel, H.; Scuseria, G.; Robb, M.; Cheeseman, J.; Scalmani, G.; Barone, V.; Mennucci, B.; Petersson, G., Gaussian 09 Revision A. 1 Gaussian Inc; 2009. Wallingford CT.

(8) (a) Grimblat, N.; Zanardi, M. M.; Sarotti, A. M., Beyond DP4: an Improved Probability for the Stereochemical Assignment of Isomeric Compounds using Quantum Chemical Calculations of NMR Shifts. J. Org. Chem. 2015, 80 (24), 12526-34; (b) Lauro, G.; Das, P.; Riccio, R.; Reddy, D. S.; Bifulco, G., DFT/NMR Approach for the Configuration Assignment of Groups of Stereoisomers by the Combination and Comparison of Experimental and Predicted Sets of Data. J. Org. Chem. 2020, 85 (5), 3297-3306.

(9) Shang, Z.; Li, L.; Esposito, B. P.; Salim, A. A.; Khalil, Z. G.; Quezada, M.; Bernhardt, P. V.; Capon, R. J., New PKS-NRPS tetramic acids and pyridinone from an Australian marine-derived fungus, Chaunopycnis sp.

Org. Biomol. Chem. 2015, 13 (28), 7795-7802. 